\title{
Secreted inhibitors drive the loss of regeneration competence in Xenopus limbs
}

\author{
Can Aztekin ${ }^{1,2, *}$, Tom W. Hiscock ${ }^{1,3,4, *}$, John Gurdon ${ }^{1,2}$, Jerome Jullien ${ }^{1,2,5, \ddagger}$, John Marioni ${ }^{3,6,7, \ddagger}$ \\ and Benjamin David Simons 1,8,9, $^{1,+}$
}

\begin{abstract}
Absence of a specialized wound epidermis is hypothesized to block limb regeneration in higher vertebrates. However, the factors preventing its formation in regeneration-incompetent animals are poorly understood. To characterize the endogenous molecular and cellular regulators of specialized wound epidermis formation in Xenopus laevis tadpoles, and the loss of their regeneration competency during development, we used single-cell transcriptomics and ex vivo regenerating limb cultures. Transcriptomic analysis revealed that the specialized wound epidermis is not a novel cell state, but a re-deployment of the apical-ectodermal-ridge (AER) programme underlying limb development. Enrichment of secreted inhibitory factors, including Noggin, a morphogen expressed in developing cartilage/bone progenitor cells, are identified as key inhibitors of AER cell formation in regeneration-incompetent tadpoles. These factors can be overridden by Fgf10, which operates upstream of Noggin and blocks chondrogenesis. These results indicate that manipulation of the extracellular environment and/or chondrogenesis may provide a strategy to restore regeneration potential in higher vertebrates.
\end{abstract}

\section{KEY WORDS: Limb regeneration, Xenopus, Apical-ectodermal- ridge, scRNA-Seq, Ex vivo limbs}

\section{INTRODUCTION}

Amphibian limb regeneration relies on a specialized wound epidermis (also known as the apical-epithelial-cap, AEC) that forms on the amputation plane and has been characterized primarily

${ }^{1}$ Wellcome Trust/Cancer Research UK Gurdon Institute, University of Cambridge, Cambridge CB2 1QN, UK. Department of Zoology, University of Cambridge, Cambridge CB2 3EJ, UK. ${ }^{3}$ Cancer Research UK Cambridge Institute, University of Cambridge, Cambridge CB2 ORE, UK. ${ }^{4}$ Institute of Medical Sciences, Foresterhill Health Campus, University of Aberdeen, Aberdeen AB25 2ZD, UK. ${ }^{5}$ Nantes Université, Inserm, Centre de Recherche en Transplantation et Immunologie, UMR 1064, ITUN, F-44000 Nantes, France. ${ }^{6}$ EMBL-European Bioinformatics Institute, Wellcome Genome Campus, Cambridge CB10 1SA, UK. ${ }^{7}$ Wellcome Sanger Institute, Wellcome Genome Campus, Cambridge CB10 1SA, UK. ${ }^{8}$ Department of Applied Mathematics and Theoretical Physics, Centre for Mathematical Sciences, University of Cambridge, Cambridge CB3 OWA, UK. ${ }^{9}$ Wellcome Trust Centre for Stem Cell Research, University of Cambridge, Cambridge CB2 OAW, UK.

*These authors contributed equally to this work

†Authors for correspondence ( jerome.jullien@inserm.fr; marioni@ebi.ac.uk; bds10@cam.ac.uk)

(D) C.A., 0000-0002-6544-9752; T.W.H., 0000-0002-0319-8679; J.G., 0000-00025621-3799; J.J., 0000-0002-7868-0021; J.M., 0000-0001-9092-0852; B.D.S., 00000002-3875-7071

This is an Open Access article distributed under the terms of the Creative Commons Attribution License (https://creativecommons.org/licenses/by/4.0), which permits unrestricted use

distribution and reproduction in any medium provided that the original work is properly attributed.

Handling Editor: Patrick Tam

Received 7 December 2020; Accepted 5 May 2021 as a tissue in regenerating salamander limbs (Campbell et al., 2011; Campbell and Crews, 2008; Knapp et al., 2013; Monaghan et al., 2012; Pearl et al., 2008; Tsai et al., 2020, 2019). It has been hypothesized that the absence or immature state of this tissue limits the regeneration potential of higher vertebrates, including mammals (Tassava and Olsen, 1982). The AEC has been suggested to impact underlying tissues by: degrading extracellular matrix (Kato et al., 2003; Miyazaki et al., 1996; Yang et al., 1999); secreting growth factors to promote proliferation (Han et al., 2001; Thornton, 1960; Thornton and Thornton, 1965; Tsai et al., 2020); enabling the selfrenewal of underlying progenitor and dedifferentiated cells, leading to the formation of a proliferative structure called the blastema (Mescher, 1976; Tassava and Loyd, 1977; Tassava and Mescher, 1975); and providing directionality cues for growth (Ghosh et al., 2008; Thornton, 1960; Thornton and Thornton, 1965). Some marker genes associated with AEC (e.g. $F g f 8$ and $F n 1)$ were seen only in the basal layers of AEC tissue, suggesting there is cellular heterogeneity within the AEC (Christensen and Tassava, 2000; Tsai et al., 2020; Yokoyama et al., 2000). However, it remains largely unclear which cell types within AEC tissue are crucial for regeneration, which transcriptional and functional properties are associated with a mature AEC and regeneration, and why the AEC cannot form or maturate in some instances and/or species.

Owing to their requirement for proximal-distal outgrowth as well as the similarity in Fgf8 expression patterns, the AEC in regenerating limbs was suggested to be analogous to the apical-ectodermal-ridge (AER), a tissue that has been well-studied during mouse and chicken limb development (Beck et al., 2009). However, current results suggest that limb regeneration-competent salamanders lack a developmental AER (Purushothaman et al., 2019). Moreover, recent findings (including single-cell transcriptomic data) have provided conflicting results on epidermal $F g f 8$ expression during axolotl limb regeneration (Gerber et al., 2018; Han et al., 2001; Leigh et al., 2018; Li et al., 2020; Nacu et al., 2016; Qin et al., 2020; Rodgers et al., 2020; Vincent et al., 2020). Therefore, it is unclear whether cells within AEC tissue use a novel transcriptional programme for regeneration, or whether they re-deploy a transcriptional programme associated with developmental AER.

Xenopus laevis is the only commonly used model organism that develops its limbs in a similar manner to amniotes, has a detectable AER and shows limb regeneration ability (Purushothaman et al., 2019). Moreover, tadpoles lose their limb regeneration ability progressively during development, coinciding with their inability to form a specialized wound epidermis, although the mechanisms of regeneration incompetence and their connection to the specialized wound epidermis remain incompletely understood (Christen and Slack, 1997; Dent, 1962). At the developmental stages prior to the formation of digits, amputations lead to a complete regeneration of the limb [Nieuwkoop and Faber stage (NF) 52-54 (Nieuwkoop and Faber, 1994), regeneration competent]. As autopod development 
proceeds, amputations result in partial regeneration, characterized by missing digits (NF $\sim 55-57$, regeneration restricted). Towards metamorphosis, amputations either cause the growth of an unpatterned spike-like cartilaginous structure without joints and muscles, or a simple wound-healing response (NF $\sim 58$ and beyond, regeneration incompetent) (Beck et al., 2009; Dent, 1962). In addition to being stage dependent, Xenopus limb regeneration competence depends on amputation position, and is reduced when amputations are performed at more proximal regions of the limb, where there are more mature chondrogenic and osteogenic cells (Nye and Cameron, 2005; Wolfe et al., 2000). Likewise, amputation through bone results in reduced regeneration compared with amputations at the joints (Nye and Cameron, 2005; Wolfe et al., 2000). Nonetheless, the association between this stage and position dependence, and regeneration competency remains unclear.

Regeneration incompetency was suggested to result from changes in mesodermal tissue, and may involve defects in patterning of the blastema (Sessions and Bryant, 1988; Yokoyama et al., 2001). In particular, the lack of activating signals (e.g. Fgf10) was proposed to prevent the formation of a specialized wound epidermis (Yokoyama et al., 2001). However, these studies were performed at the tissue level, and it remains unclear which individual cell types within the tissue are responsible for regeneration incompetency, whether intrinsic properties of mesodermal cell types fail to activate upon injury, and whether inhibitory secreted factors, rather than a lack of activating factors, plays a role in determining regeneration-outcome. Additionally, exogenous perturbations to major signalling pathways [e.g. BMP (Beck et al., 2006; Pearl et al., 2008), FGF (D'Jamoos et al., 1998) and WNT (Yokoyama et al., 2011)] have been shown to inhibit regeneration. However, it is largely unknown how these pathways endogenously influence cell types and cellular behaviours during regeneration, or how these different pathways operate in the context of cell-cell interactions that mediate regeneration. Overall, although there are numerous tissues and genes implicated in limb regeneration competency, there is currently no unifying cellular model accounting for these disparate observations.

Here, by using single-cell RNA-sequencing (scRNA-seq), we define the cellular framework of specialized wound epidermis formation during regeneration and its failure to form at later developmental stages. Then, by using scRNA-seq and ex vivo limb cultures, we reveal the crucial role of secreted inhibitory factors in determining regeneration competency, and test this phenotype by using regeneration-associated genes. Together, these findings implicate a cellular mechanism in which factors secreted during bone/cartilage formation inhibit the formation of specialized wound epidermis at later developmental stages, compromising regeneration competency.

\section{RESULTS}

\section{Single-cell RNA-seq analysis reveals cell type heterogeneity} during development and following amputation of the limb

To compare differences in AER and AEC, as well as to detail the cellular landscape of regeneration, we used single-cell transcriptomics. To characterize developmental AER and cellular changes associated with regeneration ability, we first sequenced developing intact hindlimbs at specific morphologically defined stages: NF stage $\sim 52$ (limb bud stages), NF stage $\sim 54$ (autopod forming) and NF stage $\sim 56$ (autopod formed) (Fig. 1A). Then, to evaluate regeneration-associated AEC and the cellular responses to amputations, we profiled cells from amputated limbs and their contralateral controls. Specifically, we amputated hindlimbs from presumptive knee/ankle levels for regeneration-competent tadpoles (NF stage 52-53) and ankle level for regeneration-restricted (NF stage $\sim 55-56$ ) and regeneration-incompetent (NF stage $~ 58-60$ ) tadpoles, and sequenced cells from newly-generated tissues at 5 days post-amputation (dpa) (Fig. 1B) when the specialized wound epidermis and blastema are seen morphologically (Beck et al., 2009). Contralateral developing limb buds or autopods were sequenced as controls. We did not include a contralateral control at the regeneration-incompetent stage as our dissociation protocol was unable to dissociate bone cells without compromising other tissues.

Next, we pooled the single-cell RNA-sequencing data derived from at least two replicates for each condition (Fig. S1), and corrected our atlas for cell cycle effects (Fig. S2), yielding a total of 42,348 cells (Materials and Methods; Fig. 1C,D; Figs S3 and S4; Table S1). After clustering of cells based upon their gene expression profiles, examination of multiple marker genes (Fig. S5) revealed at least 60 distinct clusters representative of putative cell types (Fig. 1C and Fig. S3), including known populations (e.g. AER cells) and potentially new and uncharacterized cell states (e.g. a Piwill ${ }^{+}$ population in the mesenchyme) (Fig. 1E). From the cell atlas, we were able to detect cell cycle differences between cell types, e.g. distal mesenchyme progenitors were more biased towards G2/M phases compared with proximal mesenchyme progenitors (Fig. S2), as reported in mouse (Boehm et al., 2010). The Xenopus limb cell atlas is accessible using an interactive platform (https://marionilab. cruk.cam.ac.uk/XenopusLimbRegeneration/).

\section{Quantitative features of AER cell formation are associated with regeneration outcome}

We then focused on the specialized wound epidermis, or AEC, that was suggested to be analogous to the AER. Although both populations are characterized by $F g f 8$ expression (Beck et al., 2009), the extent of the similarity between these cells has not been previously tested beyond assessing the similarity of expression of a small number of markers. Using our single-cell atlas, we compared the transcriptional profiles of cells that belonged to the AER (defined as $F g f 8$-expressing epidermal cells during limb development) and the AEC ( $F g f 8$-expressing epidermal cells in 5 dpa samples). Although we did see some quantitative expression differences between cells related to AEC and AER tissues (Figs S5, S6A, Table S2), they expressed many genes in common and showed a high degree of transcriptional similarity (Fig. 2A,B, Fig. S5). Consistent with this, cells related to these tissues were aggregated within a single $\mathrm{Fg} \mathrm{S}^{+}$epidermal cluster (Fig. 2B,C). Additionally, both during development and 5 days post-amputation, $F g f 8^{+}$ epidermal cells were mostly detected as a monolayer of polarized cuboidal basal cells (Fig. S7), although multilayers were seen to form in some instances (Fig. S8). This suggests that AEC and AER tissues are not homogenous in their cellular composition, and that it is only the basal cells that express the key $F g f 8^{+}$transcriptional programme. Overall, based on their transcriptomic signature, tissue localization and cellular morphology, the $\mathrm{Fg} 8^{+}$cells that compose the AEC and AER tissues are very similar. We find that the AEC tissue does not require a novel cell state, but rather a re-deployment of the transcriptional programme associated with developmental AER, albeit with a higher signalling centre potential (Fig. 2E, Fig. S6C,D). Owing to their high degree of similarity and common expression of developmental AER genes, we named all cells from the identified $\mathrm{Fgfl}^{+}$epidermal cluster as AER cells, and referred to specific samples to distinguish between cells from the regenerationassociated AEC and the developmental AER. 


\section{A}

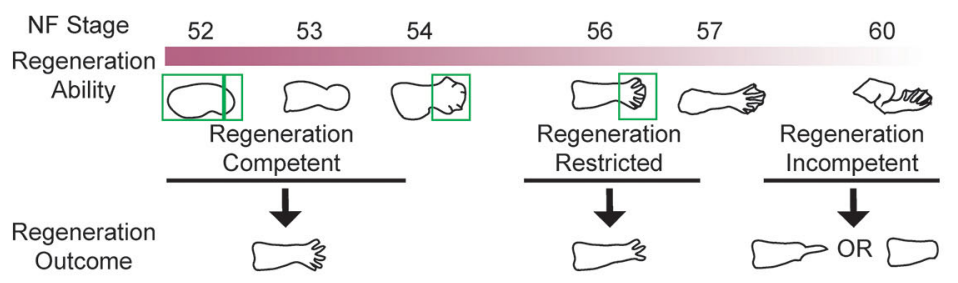

B
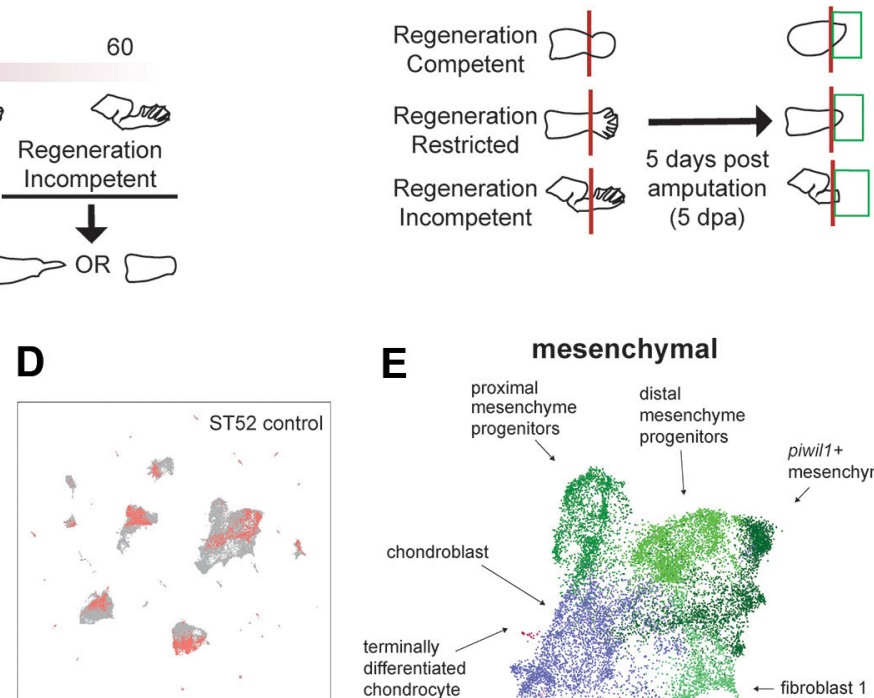

all samples selected sample

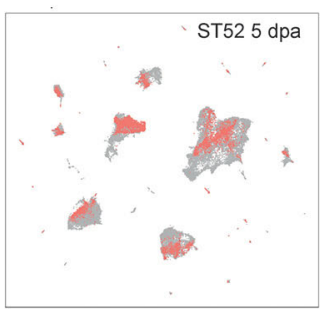

E
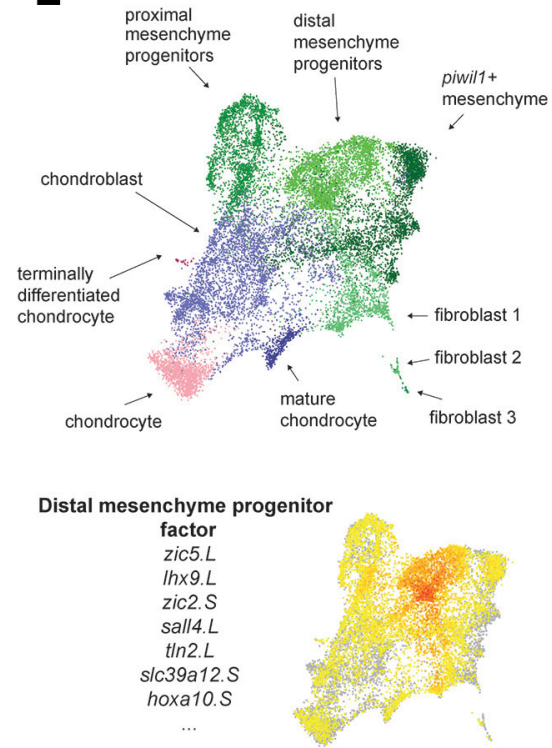

Fig. 1. Single-cell transcriptomics reveals cellular heterogeneity in developing and amputated Xenopus limbs at different stages of regeneration competence. (A) Schematic describing Xenopus limb regeneration at different NF stages. NF stage $\sim 52-54$ tadpoles are regeneration competent and amputations result in regeneration of a full limb. Regeneration ability begins to decline at NF stage $\sim 54$. Tadpoles are regeneration restricted at NF $\sim$ stage 56 , where two or three digits can be regenerated. Beyond NF stage $\sim 58$, tadpoles are regeneration incompetent and amputations result in simple wound healing or unpatterned spike formation. Green boxes indicate the location of samples collected for scRNA-seq, taken at stages prior to, at the onset of and after the loss of regeneration ability. (B) Schematic describing 5 days post-amputation (dpa) samples for regeneration-competent, -restricted and -incompetent tadpoles. Green boxes indicate the location of samples collected for scRNA-seq. (C) An atlas of cell types in intact and amputated limbs. Samples from each condition are processed separately for sequencing, and are then pooled together for UMAP visualization and clustering. Each dot corresponds to a single cell, colours indicate cluster identity, text labels important tissue/cell types. See Fig. S3 for full annotation. (D) Comparisons can be made between conditions to highlight transcriptional changes associated with regeneration; here, NF stage 52 amputated limbs (bottom) are compared with their contralateral control samples (top). Red dots indicate cells in the selected sample; grey dots indicate cells in all samples. (E) Diversity of mesenchymal cell types detected in our dataset (top), together with putative gene expression programmes identified using unbiased factor analysis (bottom).

To test the similarities of cell types composing the specialized wound epidermis in different regeneration conditions, we compared transcriptomes of cells corresponding to limb and tail specialized wound epidermis. We found that AER cells (limb-specialized wound epidermal cells) and cells that define the specialized wound epidermis during Xenopus tail regeneration (regenerationorganizing cells, ROCs; Aztekin et al., 2019) showed similar, but non-identical, gene expression profiles (Fig. S9), emphasizing that the cellular framework of the specialized wound epidermis is context dependent and appendage regeneration scenarios can use different cell types.

Limb amputation is known to result in the formation of a Fgf8expressing $\mathrm{AEC}$ at the amputation plane in regeneration-competent tadpoles, but not in regeneration-incompetent tadpoles (Christen and Slack, 1997), whereas AEC formation has not been characterized previously for regeneration-restricted tadpoles. Using our atlas, we found that, at $5 \mathrm{dpa}$, tadpole epidermis contained abundant AER cells in regeneration-competent tadpoles and a limited number of
AER cells in regeneration-restricted tadpoles, whereas AER cells were largely absent from regeneration-incompetent tadpoles (Fig. 2B-D). In parallel, AER cell-associated ligand expression was lower or absent in regeneration-incompetent $t p 63^{+}$epidermal cells (Fig. S6E). In our dataset, we found that different populations express ligands from different major signalling pathways (FGF, BMP, WNT, DELTA and TGF $\beta$ ) (Fig. S6C). However, only AER cells can express multiple ligands from these gene families concurrently and at a very high level, making them a highly potent signalling centre (Fig. 2E, Fig. S6). Although $F g f 8$ was always expressed in AER cells, the relative expression of $F g f 8$ and other ligands varied among conditions (Fig. 2E, Fig. S6, Table S2), emphasizing that the detection of $F g f 8$ alone does not discriminate the signalling centre potency of AER cells. Indeed, in addition to the changes in AER cell abundance, we also detected differentially expressed genes between AER cells from regeneration-competent and -restricted tadpoles (Fig. S6B). These differences suggest that AER cells in regeneration-competent 5 dpa samples may be more 
A

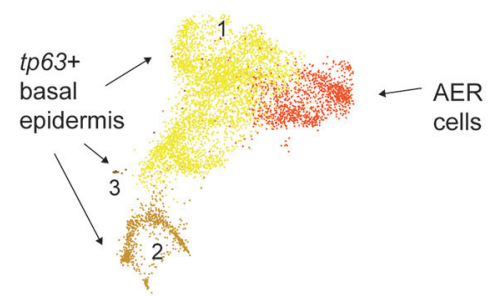

B

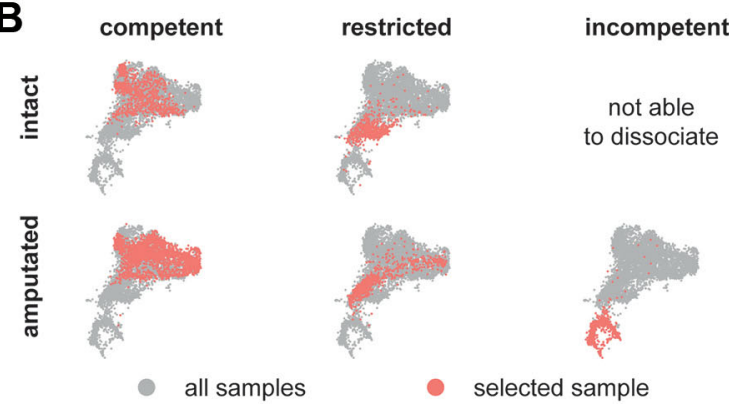

C

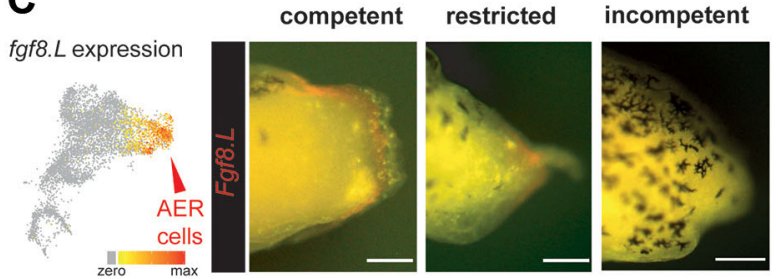

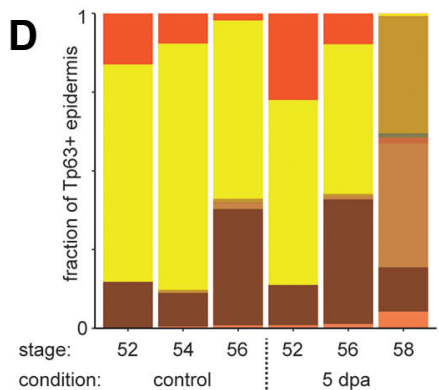

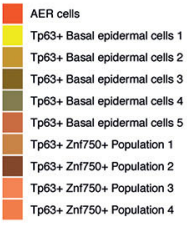

$E$

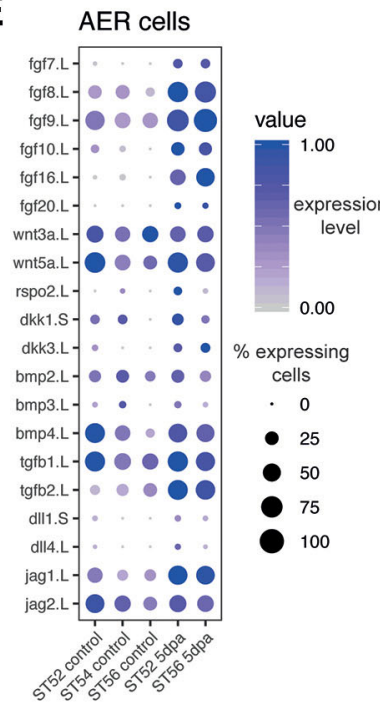

Fig. 2. Formation of a signalling centre comprising apical-ectodermal-ridge (AER) cells is associated with the successful regeneration. (A) Multiple basal epidermal cell states are detected, including AER cells, in the pooled dataset. (B) UMAP visualization of basal epidermis reveals that re-establishment of AER cells is associated with successful regeneration. Red dots indicate cells in the selected sample; grey dots indicate cells in all samples. (C) Left: UMAP visualization of pooled data for AER cells expressing Fgf8.L. Right: stereomicroscope images of the 5 dpa amputation plane of regeneration-competent, -restricted and -incompetent tadpoles. Fgf8.L-expressing AER cells (red) are formed in regeneration-competent and -restricted tadpoles, but not in regenerationincompetent tadpoles. Scale bars: $250 \mu \mathrm{m}$. (D) The abundance of basal epidermal cell types across conditions reveals a correlation between AER abundance and regeneration outcome. AER cells are present in intact regeneration-competent samples, and are enriched after amputation. A similar pattern is seen in regeneration-restricted samples,

although abundances of AER cells are reduced. Very few AER cells are detected in

regeneration-incompetent tadpoles. (E) Dot plot showing expression of selected ligands for AER cells during development and at $5 \mathrm{dpa}$ in regeneration-competent and -restricted samples. Dot colour indicates mean expression; dot size represents the percentage of cells with non-zero expression. 'mature' compared with regeneration-restricted cells, although further work on the functional role of these genes (e.g. Sesn1.L, Cpn1.S, Ddx21.L) is required. Overall, although the signalling centre potency of AER cells appeared variable, the redeployment of this developmental cell type with a high signalling centre potential had a strong correlation with regeneration outcome.

\section{The presence of AER cells is associated with injury-induced mesenchymal plasticity}

It has been suggested that the AEC enables the self-renewal activity of dedifferentiated cells, leading to blastema formation (Tassava and Mescher, 1975; Tassava and Loyd, 1977). To identify signatures of dedifferentiation in our atlas, we first examined the expression of genes related to dedifferentiation and blastema formation (Gerber et al., 2018; Haas and Whited, 2017; Leigh et al., 2018) (e.g. Sall4 and Kazald1). We found that these genes were either already expressed before amputation or upregulated upon amputation in a subset of fibroblasts (Fig. S10A,B) that were located near the skin and perichondrium (Fig. S11). Likewise, we found that a small fraction of these fibroblasts expressed muscle-related genes (e.g. Pax3) before and after amputation (Fig. S10B). Moreover, independently of regeneration outcome, amputation resulted in these fibroblast cells expressing genes related to distal mesenchyme progenitors (e.g. Grem 1, Shh, Msxl and Fgf10) and chondrogenesis (e.g. Col8a2 and Sox9) (Fig. S10A). Finally, amputation not only increased the expression of known marker genes, but also led to the upregulation of an entire putative distal mesenchyme progenitor gene set (Fig. S10C), with the magnitude of this upregulation being lower in samples that had fewer AER cells. Together, we concluded that, upon amputation, a subset of fibroblasts manifest injury-induced mesenchymal plasticity - at least at the transcriptional level - and its extent correlates with AER cell abundance.

\section{AER cell formation requires activation of multiple signalling pathways}

To investigate the molecular mechanisms that mediate AER cell formation upon amputation, we developed an ex vivo regenerating limb culture protocol, inspired by previous work (Cannata et al., 1992) (Fig. 3A). By culturing amputated stylopod, or zeugopod and stylopod from regeneration-competent or regeneration-restricted tadpoles, respectively, we observed $F g f 8$ cell formation at the distal part of explants within 3 dpa (Fig. 3B). Regeneration-competent explants also exhibited cone-shaped growth as cells accumulated uniformly underneath $F g f 8$ cells, mimicking in vivo regeneration (Fig. 3A,B, Fig. S12A,B). Interestingly, the proximal region of explants was also covered with epidermis (Figs S12A, S13A), but neither $F g f 8$-expressing cells nor a uniform cell accumulation underneath the epidermis was observed (Fig. 3A,B, Figs S12B, S13A). Moreover, the proximal part of the explant exhibited active chondrogenesis, manifesting in an outwards growth of cartilaginous tissue (Fig. 3A and Fig. S12C). This phenotype was particularly pronounced when explants were harvested from developmental stages in which proximal tissues were advanced in chondrogenesis (onset of NF stage 53-54) (Fig. 3A and Fig. S12D), and could be further enhanced by addition of BMP4, a known chondrogenesis inducer (Fig. S12E). Hence, the proximal and distal sites of limb explants exhibit different behaviours: the distal sites recapitulate localized AER cell formation, as seen in vivo, whereas the proximal site is characterized by active chondrogenesis without AER cell formation. 

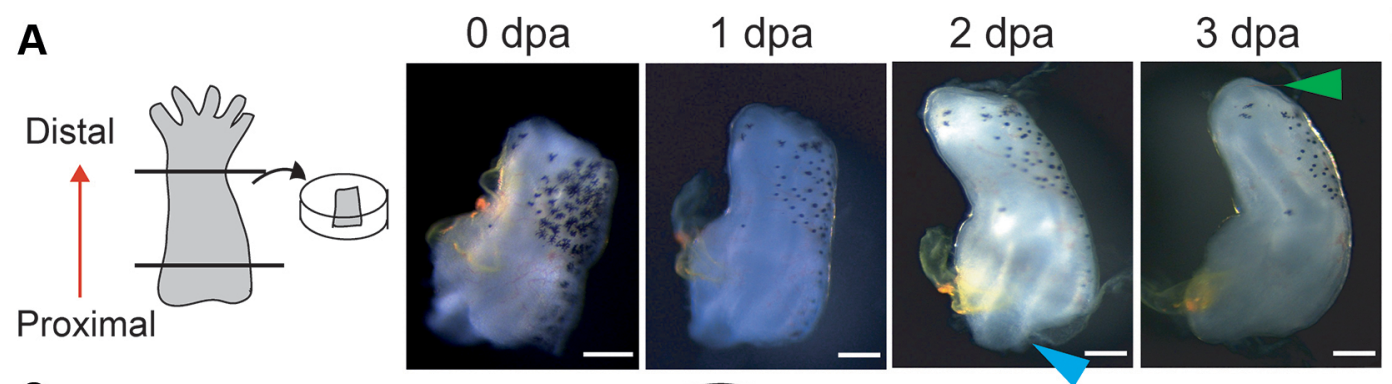

B

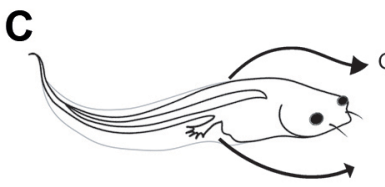

Contralateral Limb
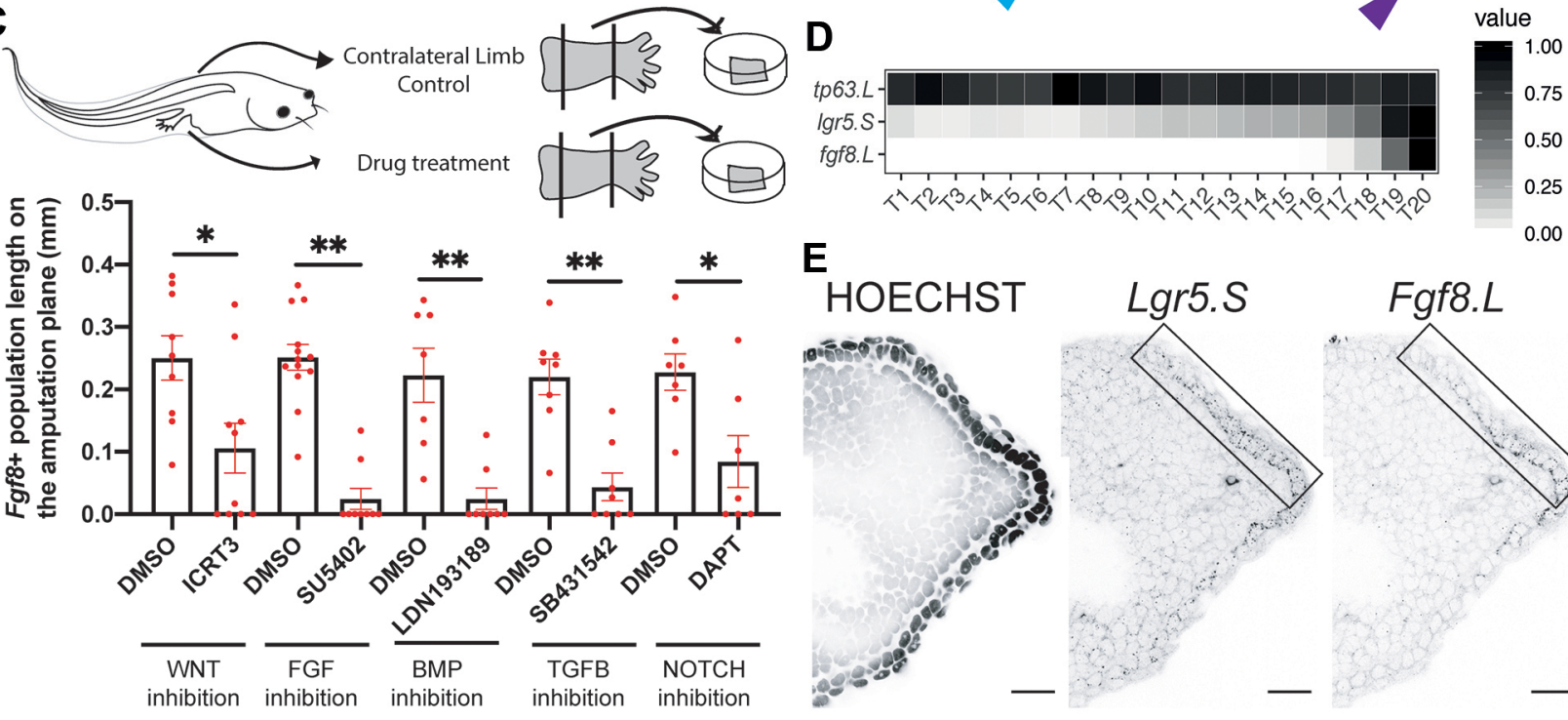

E
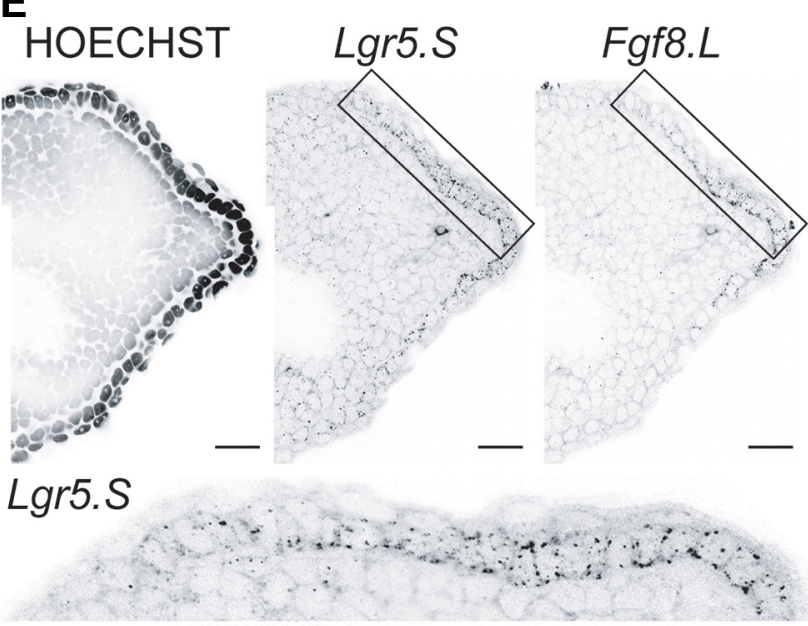

Fgf8.L

Fig. 3. Ex vivo regenerating limbs demonstrate that AER cell formation requires activation of multiple pathways and can form from basal epidermal cells. (A) Left: schematic for ex vivo regeneration limb culture. Right: time-lapse images of a regeneration-competent explant. The explant grows a cone shape at its distal site reminiscent of in vivo regeneration (green arrowhead), while the proximal site shows chondrogenesis (blue arrowhead). Scale bars: $200 \mu \mathrm{m}$. (B) An example image of a regeneration-competent explant at 3 days post-culture. The distal site of explants is Fgf8.L positive (red arrowhead); the proximal site is Fgf8.L negative (purple arrowhead). Red, Fgf8.L mRNA. Scale bar: $200 \mu \mathrm{m}$. (C) Drug screen to test regulators of AER cell formation. Top: schematics describing the screen. One limb of a tadpole was used for perturbation and the contralateral limb from the same tadpole was used as a control. Samples were treated with the indicated drugs for 3 days post-culture, and then stained for Fgf8.L mRNA. Bottom: the extent of Fgf8.L expression along the amputation plane was measured. Sample sizes: ICRT3 total, $n \geq 9$ from three biological replicates; SU5402 total, $n \geq 9$ from two biological replicates;

LDN193189 total, $n=8$ from three biological replicates; SB431542 total, $n=8$ from two biological replicates; DAPT total, $n=7$ from three biological replicates. ${ }^{*} P<0.05,{ }^{*} P<0.001$. Data are mean \pm s.e.m. (D) Factor analysis identifies a putative gene expression trajectory from basal epidermal cells to AER cells, predicting sequential activation of Lgr5.S followed by Fgf8.L. (E) A proximal-to-distal gradient of Lgr5.S and Fgf8.L is observed in vivo, with Fgf8.L being restricted to the most distal regions of the midline epidermis. Black dots represent HCR mRNA signal. Scale bars: $20 \mu \mathrm{m}$.

In addition to changes associated with regeneration, explants could be used to determine signalling requirements for specialized wound epidermis formation. Inhibition of FGF, BMP and WNT pathways via small molecule inhibitors blocked AER cell formation in explants (Fig. 3C), reinforcing the conclusion that the in vivo AEC effects reported in former studies are mediated through a direct effect on the limb rather than a systemic effect (Beck et al., 2006; D'Jamoos et al., 1998; Yokoyama et al., 2011). Moreover, by using the culture assay, we found that active TGF $\beta$ and NOTCH signalling are also required for Xenopus AER cell formation (Fig. 3C). Overall, we concluded that AER cell formation requires the activity of multiple major signalling pathways, although further work is required to determine what roles these pathways play and whether they directly or indirectly regulate AER cell formation.

\section{AER cells can form without cell division}

Next, we asked how AER cells form on the amputation plane. It has been suggested that salamander AEC tissue forms by migration of epidermal cells to the amputation plane, and may not require cell proliferation (Campbell and Crews, 2008; Hay and Fischman, 1961). Moreover, the mouse AER was previously suggested to be a largely mitotically inactive tissue (Storer et al., 2013). However, it is not known whether similar mechanisms apply to AER cells within the specialised wound epidermis, and also to what extent they are 
seen in Xenopus. Therefore, we first traced skin tissue located on the edge of explants, and found that they contributed to the covering of both the distal and proximal sites (Fig. S13B). As the amputation planes are covered by skin tissue from the surrounding area, we reasoned that AER cells are likely to have originated from skin cells. As amputation eliminates the majority, if not all, of AER cells in the limb, we hypothesized that AER cells are derived from remaining skin stem cells. If AER cells are induced through proliferation and differentiation following amputation, all AER cells should be the product of cell division. To test this hypothesis, we assayed the level of EdU incorporation (labelling newly synthesized DNA, hence divided cells) in newly formed AER cells, using Fgf8 positivity to specifically identify AER cells within the AEC tissue. We found that only $\sim 40 \%$ of AER cells (distal epidermal $\mathrm{Fgff}^{+}$) were EdU positive at 3 dpa (Fig. S13C), suggesting that most AER cells are induced independently of cell division following amputation. These results parallel our transcriptomics-based cell-cycle assessment in which AER cells display low levels of proliferation (Fig. S2D). Using the transcriptomics data, we identified a stepwise activation of Lgr5.S (a WNT target gene) followed by Fgf8.L expression as a possible gene-expression trajectory that could allow basal epidermal cells to convert directly to AER cells without cell division (Fig. 3D). Consistent with such a process, when visualized in vivo, we found that $\mathrm{Fg} f 8^{+} / \mathrm{Lgr} 5^{+}$AER cells were flanked by $\operatorname{Lgr} 5^{+}$cells in the basal epidermis on the amputation plane or in the developing limb (Fig. 3E and Fig. S7A,B). Overall, these results support the hypothesis that basal epidermal cells can acquire AER cell identity without cell division, although understanding the functional relevance of cell division on AER cell fate requires further work.

\section{Loss of regeneration potential is associated with enrichment in inhibitory secreted factors to AER cell formation}

We then asked why fewer or no AER cells form on the amputation plane of regeneration-restricted or -incompetent tadpoles, respectively. Previous studies have proposed that lack of activating signals in the mesodermal tissue, specifically $F g f 10$, causes regeneration incompetency (Yokoyama et al., 2001). However, these results cannot explain why regeneration is impaired when amputations are conducted through bone or at more-proximal limb regions, or why the proximal site of limb explants cannot form AER cells. Thus, we assessed whether $F g f 10$ can induce $F g f 8$ expression across the whole epidermis or whether there are additional requirements for distal epidermal $F g f 8$ expression, and hence AER cell formation.

When we examined the spatial correlation between Fgf10.Lexpressing mesenchymal cells and Fgf8.L-expressing epithelial cells in regeneration-competent tadpoles, we saw regions in which Fgf10.L but not Fgf8.L was present (Fig. S14A). Second, when adding FGF10 to regeneration-competent explants, we observed a slight, but not statistically significant, increase in AER cell formation on the amputation plane (Fig. S14B); however, this signal was confined to the distal epidermis and did not include a substantial signal at the proximal site of explants (Fig. S14C), where chondrogenic populations are more abundant. This suggested that FGF10 alone cannot induce AER cell formation across the entire epidermis, and that the presence/absence of further activating/ inhibitory signals are involved in AER cell formation.

To test whether there are inhibitory factors secreted from regeneration-incompetent tadpole limbs that block AER cell formation, we took advantage of our ex vivo cultures. First, we co-cultured ex vivo limbs from regeneration-competent and -incompetent tadpoles. Strikingly, when such cultures were stained against $\mathrm{Fg} f 8$ at $3 \mathrm{dpa}$, we observed that regeneration- competent tadpole limbs failed to form AER cells (Fig. 4A). Second, we collected media from regeneration-incompetent tadpole explants and cultured freshly amputated regeneration-competent explants with this conditioned media. Consistent with the co-culture experiment, the conditioned media from regeneration-incompetent tadpoles blocked AER cell formation in regeneration-competent explants (Fig. 4B). By contrast, neither co-culturing with regeneration-competent explants nor preparing conditioned media from regeneration-competent explants affected AER cell formation in regeneration-competent explants (Fig. 4A,B). Additionally, conditioned media from regeneration-competent explants was unable to induce AER cell formation in regeneration-incompetent explants (Fig. S15A,B), emphasizing that it is the enrichment of inhibitory secreted factors that is the dominant process interfering with AER cell formation, rather than a depletion in regenerationpromoting factors. Altogether, these results suggest that secreted inhibitory factors block AER cell formation in regenerationincompetent tadpoles, presumably compromising their regeneration potential.

To identify the factors responsible for this inhibitory effect, we surveyed our single-cell atlas for the expression of secreted proteins involved in signalling pathways required for AER cell formation. We found that that the loss of regeneration potential is associated with an increased proportion of chondrogenic lineage cells in the mesenchyme [Fig. 4C, aligning with previous tissue-level observations (Dent, 1962)] and that these cells express multiple inhibitory ligands for BMP and WNT pathways (Fig. 4D). As chondrogenic populations specifically express high levels of Noggin (Fig. 4D), a known antagonist of BMP signalling, we hypothesized that AER cell formation is antagonized by an excess of secreted Noggin in regeneration-incompetent tadpoles. Indeed, consistent with previous observations (Pearl et al., 2008), addition of NOGGIN to regeneration-competent ex vivo limbs blocked AER cell formation (Fig. S15C). To test whether endogenous NOGGIN does indeed act as one of the inhibitory secreted factors produced following amputation in regeneration-incompetent tadpoles, we blocked NOGGIN in our co-culture and conditioned media experiments using anti-NOGGIN antibodies (Fig. 4A,B). Strikingly, blocking secreted NOGGIN by antibody addition cancelled the inhibitory activity on AER cell formation in both co-culture and conditioned media experiments (Fig. 4A,B). Based on these observations, we then explored whether anti-NOGGIN application would improve the in vivo amputation response. Indeed, when beads loaded with anti-NOGGIN antibodies were implanted on the amputation plane of regeneration-restricted/incompetent tadpoles, we saw a mild improvement in the regenerative response (Fig. 5A), highlighting that secreted inhibitors are influencing the regenerationoutcome in vivo.

As these experiments point towards the chondrogenic lineage as the source of inhibitory secreted factors, we then asked whether limiting chondrogenesis can promote AER cell formation. To this end, we generated tip explants by culturing distal limb buds (NF stage $\sim 52$ ) or early formed autopods (NF stage $\sim 54$ ) without their proximal segment, where the most advanced chondrogenesis takes place. Indeed, these tip explants showed ectopic $F g f 8$ expression at different sites of the epidermis, further suggesting a localized and/or long-range inhibitory effect of secreted factors from mature chondrogenic cells (Fig. S15D). Moreover, the inability of the proximal explant epidermis to form AER might be explained, at least in part, by the abundance of chondrogenic cells at the proximal site (Fig. 3A,B). Overall, these results indicate that the loss of AER cell formation ability is associated with an enrichment in inhibitory 
A

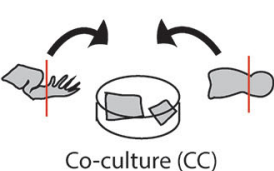

-competent and -incompetent

w/wo anti-Noggin antibody
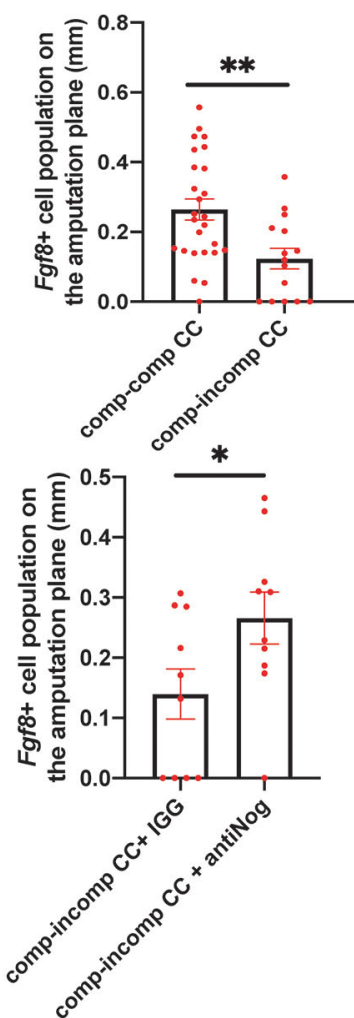

B

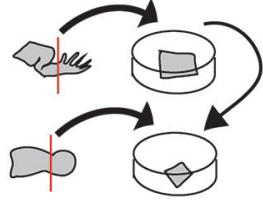

Conditioned media (CM) w/wo anti-Noggin antibody
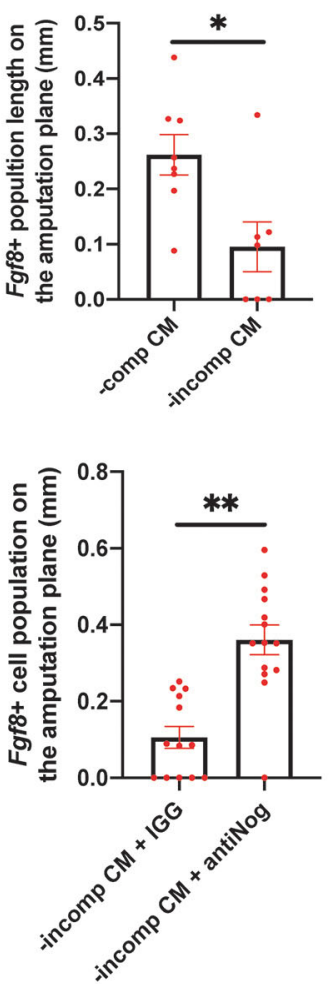
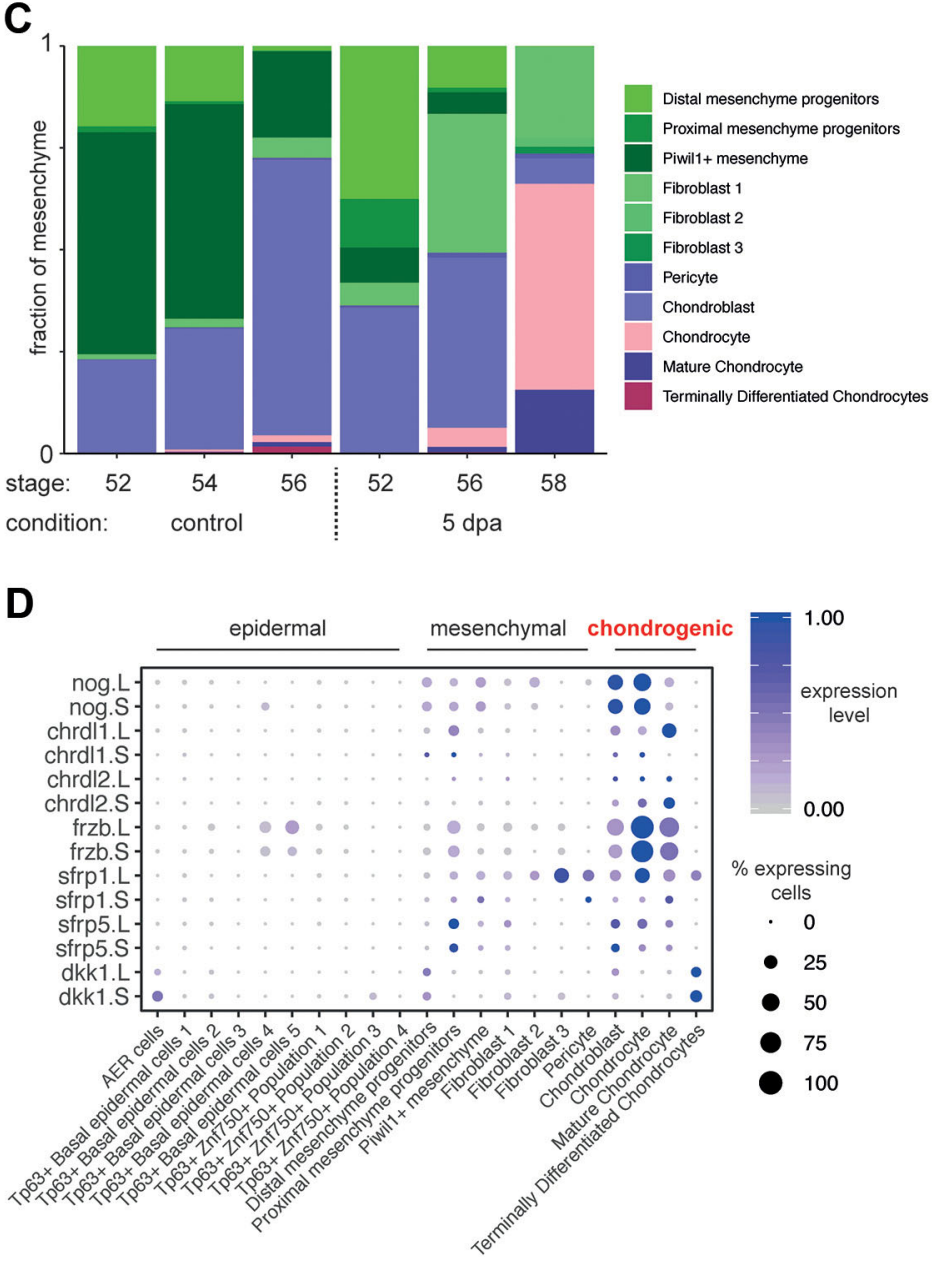

Fig. 4. Inhibitory factors, such as Noggin, are secreted from chondrogenic populations at regeneration-incompetent stages and block AER cell formation. (A) Top: schematic describing co-culture experiments. Middle: co-culturing regeneration-competent and -incompetent explants decreases the extent of Fgf8.L expression at the amputation plane at $3 \mathrm{dpa}$. Bottom: this effect can be rescued by adding anti-NOGGIN antibody. Regeneration-competent and -competent co-culture total, $n=26$ from four biological replicates; regeneration-competent and -incompetent co-culture total, 15 from four biological replicates; competent and incompetent co-culture, and anti-IGG antibody total, $n=10$ from three biological replicates; competent and incompetent co-culture, and anti-NOGGIN antibody total, $n=10$ from three biological replicates. ${ }^{*} P<0.05$ and ${ }^{* *} P<0.001$. Data are mean \pm s.e.m. (B) Top: schematic describing conditioned media experiments to test the effect of secreted factors in regeneration-incompetent tadpole limbs. Middle: supplying conditioned media (CM) from regeneration-incompetent tadpoles to regeneration-competent explants decreases the extent of Fgf8.L expression at the amputation plane at 3 dpa. Bottom: this effect can be rescued by adding anti-NOGGIN antibody. Regeneration-competent CM to -competent explants total, $n=8$ from three biological replicates; regeneration-incompetent $\mathrm{CM}$ to -competent explants total, $n=7$ from three biological replicates; regeneration-incompetent $\mathrm{CM}$ to -competent explants, and anti-IGG antibody total, $n=10$ from three biological replicates; regeneration-incompetent CM and anti-NOGGIN antibody to regenerationcompetent explants total, $n=10$ from three biological replicates. ${ }^{*} P<0.05$ and ${ }^{* *} P<0.001$. Data are mean \pm s.e.m. (C) Abundance of mesenchymal populations across conditions reveals an enrichment of chondrogenic populations at regeneration-restricted and -incompetent stages, in both intact and amputated limbs. (D) Multiple BMP/WNT antagonists are expressed specifically in chondrogenic populations. This dotplot is generated using the pooled dataset, with latestage tadpoles having high levels of chondrogenic and fibroblast populations, but not immature mesenchymal cell types, as shown in C.

secreted factors, including NOGGIN, that are secreted primarily from the chondrogenic lineage.

As NOGGIN is known to neutralize secreted BMPs, we then focused on assessing the effect of the BMP pathway on AER cell formation. Previously, it was demonstrated that, not only do mouse and chicken AER require active BMP signalling, but also excess BMP activation abolishes AER (Pajni-Underwood et al., 2007; Pizette et al., 2001; Pizette and Niswander, 1999; Verheyden and Sun, 2008). To test whether manipulation of BMP signalling can also impact Xenopus AER cell formation in regeneration-competent tadpoles, we perturbed the BMP pathway. We found that the addition of BMP4 to regeneration-competent ex vivo cultures blocked AER cell formation
(Fig. S15C), an effect similar to that reported in chick and mouse embryos (Pajni-Underwood et al., 2007; Pizette et al., 2001; Pizette and Niswander, 1999; Verheyden and Sun, 2008). The addition of NOGGIN to regeneration-competent ex vivo cultures blocked AER cell formation (Fig. S15C), as reported before in vivo (Beck et al., 2006; Jones et al., 2013). Moreover, we found that inhibiting NOGGIN could increase the formation of AER cells (Fig. S15C), suggesting endogenous BMP4 levels do not reach a level where AER cell formation is blocked. As BMP4 boosts chondrogenesis (Fig. S12E), which can, in turn, lead to Noggin expression, these results suggest that fine-tuning of BMP agonist and antagonists levels in the growing limb are key for AER cell formation. 
A

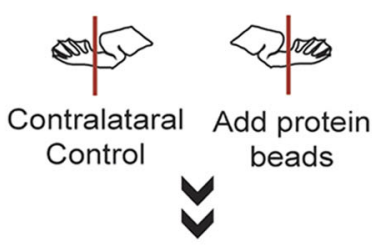

Assess digit formation and phenotypes

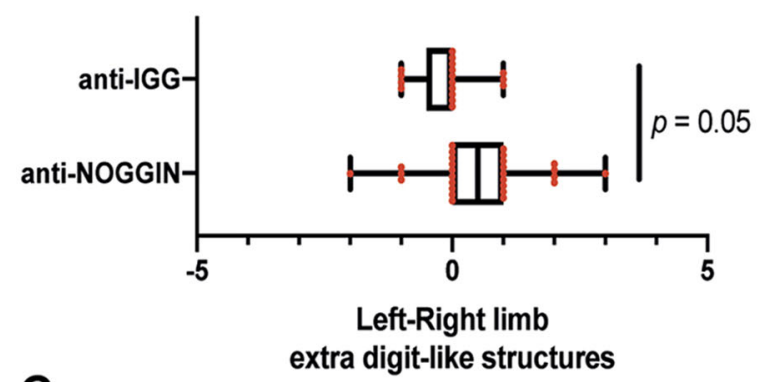

C

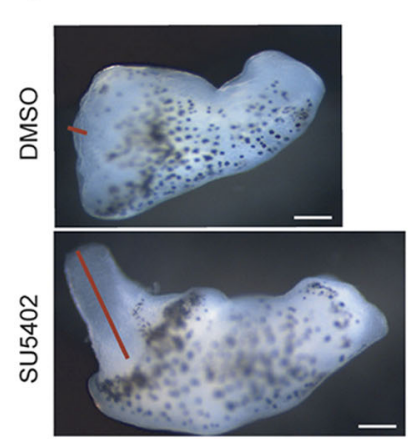

E

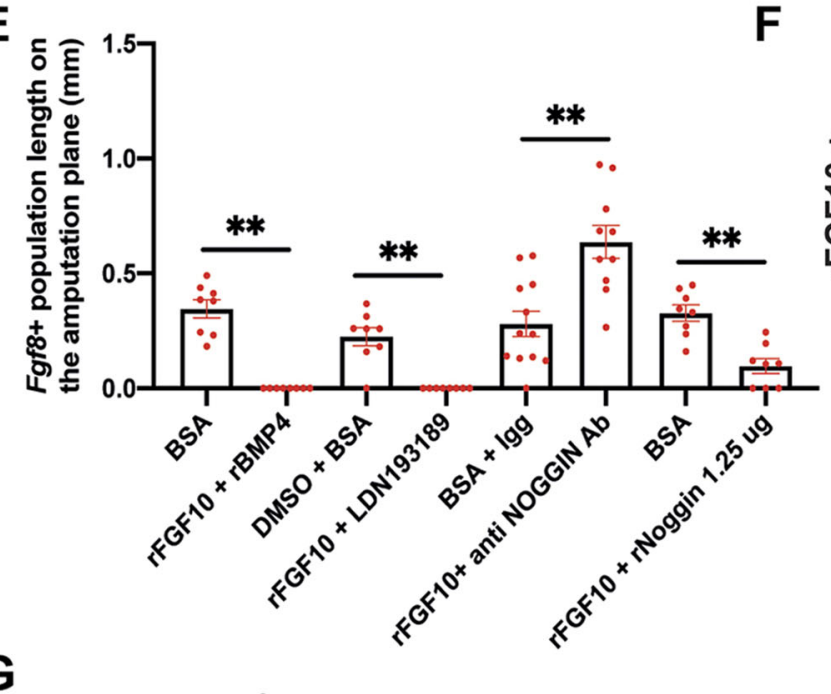

G
B
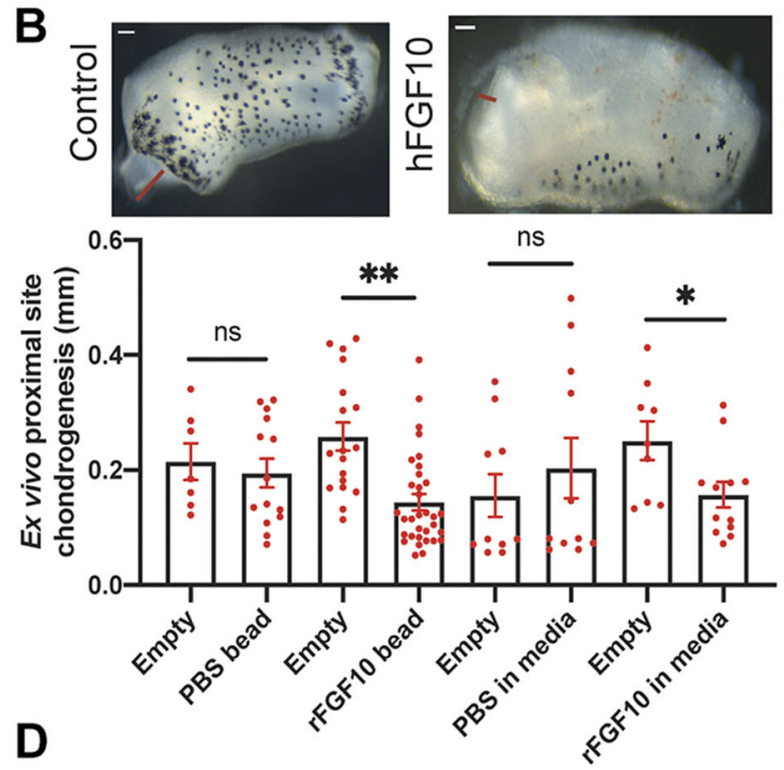

D
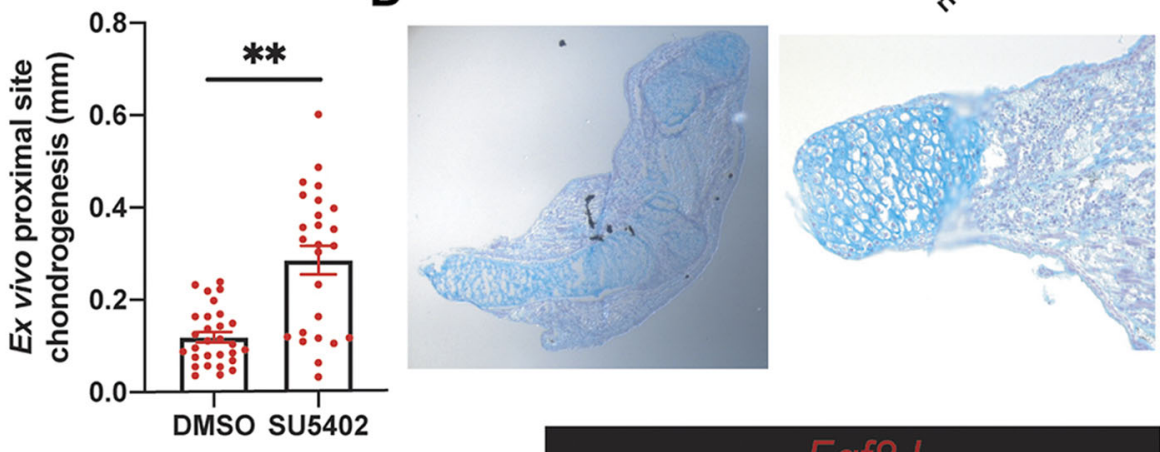
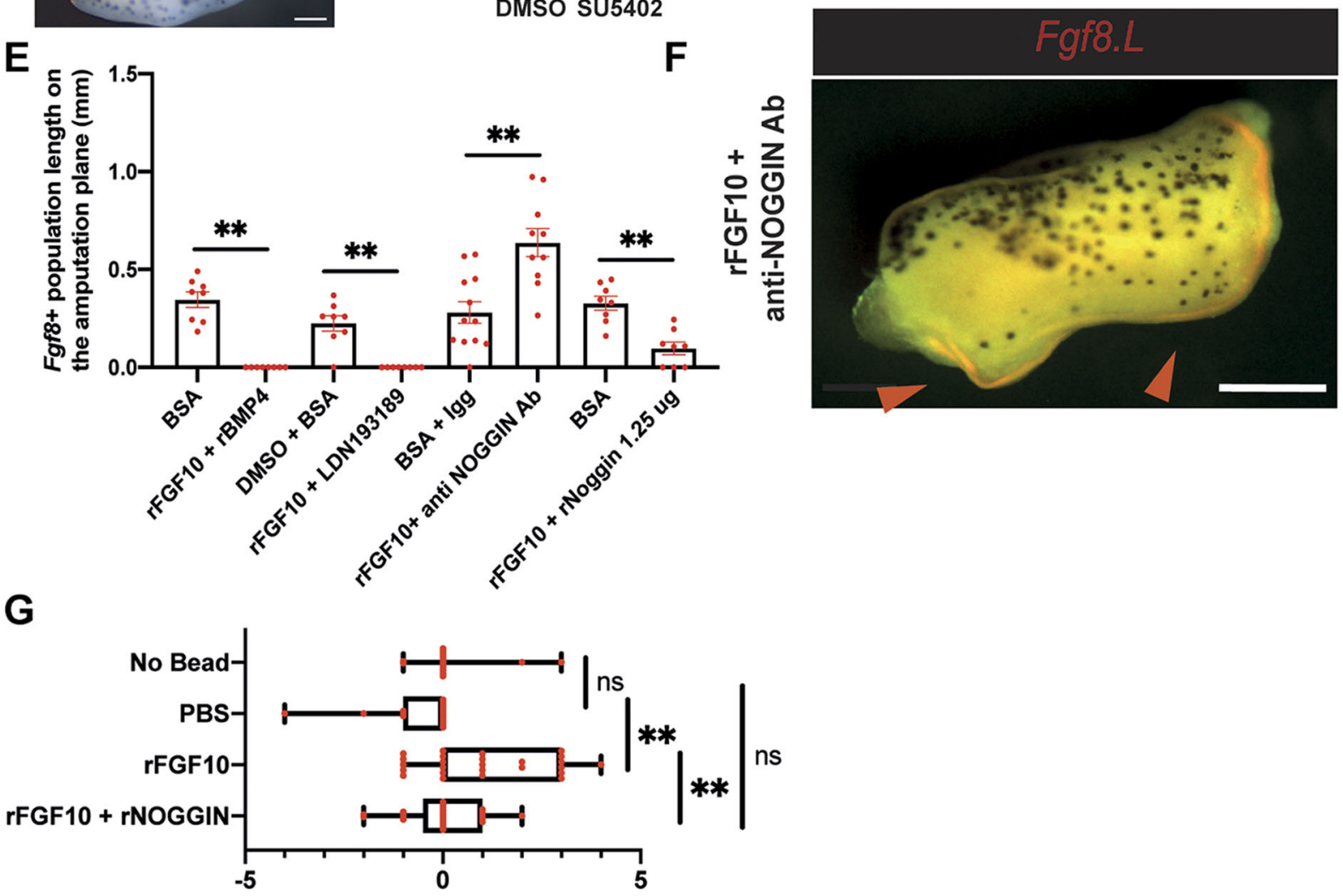

Left-Right limb

extra digit-like structures

Fig. 5. See next page for legend. 
Fig. 5. FGF10 impacts chondrogenesis and operates upstream of NOGGIN. (A) Anti-NOGGIN antibody application to distal amputations improves regeneration in regeneration-restricted and -incompetent tadpoles. Regeneration-restricted and -incompetent tadpole right and left hindlimbs were amputated, and beads containing anti-IGG antibody or anti-NOGGIN antibody were placed on the right hindlimbs. Formed digits and digit-like structures were quantified in the right and left hindlimbs, and the difference calculated. Anti-IGG antibody total, $n=17$ from three biological replicates; anti-NOGGIN antibody total, $n=28$ from four biological replicates. (B) The effect of FGF10 on chondrogenesis is assessed by measuring the chondrogenic outgrowth at the proximal sites of regeneration-restricted explants at $3 \mathrm{dpa}$. Red lines show measured proximal chondrogenesis. Implanting $0.1 \%$ BSA/PBS beads at the proximal site or supplying $0.1 \%$ BSA/PBS to the media had no significant effect on chondrogenesis, while implanting Fgf10 beads at the proximal site or supplying FGF10 in media reduced chondrogenesis. Contralateral limbs were used as controls and are labelled as empty. From left to right, empty and PBS beads total, $n \geq 7$ from at least two biological replicates; empty and FGF10 bead total, $n \geq 14$ from at least four biological replicates; empty and 0.1\% BSA/PBS in media total, $n=10$ from three biological replicates; empty and FGF10 in media, $n \geq 14$ from at least three biological replicates. ns, not significant; ${ }^{*} P<0.05$ and ${ }^{* *} P<0.001$. Data are mean \pm s.e.m. Scale bar: $50 \mu \mathrm{m}$. (C) Left: example images of SU5402-treated explants showing extensive chondrogenesis at the proximal site. Red lines show measured proximal chondrogenesis. Right: blocking FGFR via the small molecule inhibitor SU5402 extends chondrogenesis in 3 days for regeneration-competent and -restricted explants. Contralateral limbs were used as controls and treated with DMSO. DMSO total, $n=29$ from seven biological replicates; SU5402 total, $n=25$ from seen biological replicates. ${ }^{* *} P<0.001$. Data are mean \pm s.e.m. Scale bar: $200 \mu \mathrm{m}$. (D) Representative sectioned histology images for 3 dpa explants treated with SU5402. The outgrowing structures are Alcian Blue rich, which is indicative of chondrogenic cells. (E) Regeneration-competent explants were treated with a combination of FGF10 and recombinant BMP4, or with recombinant NOGGIN, with LDN193189 or with anti-NOGGIN antibody. $0.1 \% \mathrm{BSA} / \mathrm{PBS}$ and anti-IGG antibody were used as controls. From left to right: BSA total, $n=8$ from two biological replicates; recombinant FGF10 and recombinant BMP4 total, $n=8$ from two biological replicates; DMSO and BSA total, $n=8$ from two biological replicates; FGF10 and LDN total, $n=8$ from two biological replicates; BSA and anti-IGG antibody total, $n=12$ from three biological replicates; FGF10 and anti-NOGGIN antibody total, $n=10$ from three biological replicates; BSA total, $n=8$ from two biological replicates; recombinant FGF10 and recombinant NOGGIN total, $n=8$ from two biological replicates. ${ }^{* *} P<0.001$. Data are mean \pm s.e.m. (F) Representative wholemount stereomicroscope image of rFGF10 and anti-NOGGIN antibody treated explants can show a substantial Fgf8.L expression at different sites of the explant ( $n=5 / 9$ from two biological replicates, compared with $n=0 / 121$ in controls, $P<0.0001)$. Scale bar: $200 \mu \mathrm{m}$. (G) Recombinant FGF10 application to distal amputations restore regeneration in -regenerationrestricted and -incompetent tadpoles. Regeneration-restricted and -incompetent tadpole right and left hindlimbs were amputated, and beads containing $0.1 \%$ BSA/PBS or recombinant FGF10 or recombinant FGF10 and NOGGIN were placed on the right hindlimbs. Formed digits and digitlike structures were quantified in the right and left hindlimbs and the difference calculated. Box and whisker plots show minimum to maximum for all data points. Red dots represent individual data points. Where applicable, boxes show interquartile range (25-75\%). Mean for No bead: 0.15; PBS: -0.5; rFGF10: 1.04; rFGF10 + rNOGGIN: 0. Empty total, $n=19$ from two biological replicates; $0.1 \% / P B S$ bead total, $n=17$ from five biological replicates; recombinant FGF10 bead total, $n=25$ from five biological replicates; recombinant FGF10 and NOGGIN bead total, $n=25$ from four biological replicates. ns, not significant; ${ }^{* *} P<0.001$

\section{FGFR activation negatively regulates progression of chondrogenesis and the FGF pathway operates upstream of NOGGIN for AER cell formation}

As regeneration competency in late stage tadpoles has bee shown previously to be restored via exogenous application of FGF10 (Yokoyama et al., 2001), we next sought to evaluate whether the effect of FgflO on regeneration is, at least in part, mediated by its impact on chondrogenesis and Noggin expression. To test the effect of FgflO on chondrogenesis, we used our ex vivo cultures to monitor the substantial chondrogenesis occurring at the proximal site of explants. Application of FGF10 beads to the proximal site of ex vivo cultures, or addition of recombinant FGF10 to their media, significantly decreased chondrogenesis at the proximal sites in regeneration-restricted explants (Fig. 5B). Conversely, blocking FGFR significantly extended chondrogenesis at the proximal site of explants (Fig. 5C-D, Fig. S16). Nonetheless, FGF10 treatment was not sufficient to induce strong Fgf8 expression at the proximal site of explants (Fig. S14C), which we hypothesize could be, at least in part, attributable to differences in the abundance of proposed antagonist cues. To test this hypothesis, we treated explants with a combination of FGF10 and anti-NOGGIN antibodies. Strikingly, this combination not only enhanced AER cell formation at the distal sites, but also induced ectopic Fgf8.L expression near the proximal sites of explants (Fig. 5E,F), further suggesting that the enrichment of inhibitory secreted factors from the chondrogenic lineage affects the ability to form AER cells. Finally, AER cell formation induced by FGF10 addition was cancelled by the addition of BMP inhibitors (NOGGIN or small molecule inhibitors) (Fig. 5E), suggesting that FGF10 acts upstream of the effect of NOGGIN ex vivo. To further test this finding in vivo, we asked whether the positive effect of FGF10 in regeneration-incompetent tadpoles could be abrogated by simultaneous NOGGIN addition. To investigate this, we inserted beads co-loaded with FGF10 and NOGGIN to the amputation plane of regeneration-restricted and/or -incompetent tadpoles, and found this significantly decreased the positive effect of FGF10-only beads (Fig. 5G). These results further emphasise that FGF10 operates upstream of NOGGIN, and hence that secreted inhibitors play a dominant role in determining regeneration outcome.

\section{DISCUSSION}

Limb regeneration and its requirement for a mature specialized wound epidermis (the AEC) is a well-established phenomenon with extensive tissue and gene level investigations. Here, moving beyond tissue-level descriptions, we reveal cell types and transcriptional states that mediate Xenopus limb regeneration and AEC tissue by using single-cell transcriptomics and ex vivo regenerating limb cultures. Transcriptome and morphological assessment indicate that the transcriptional programmes and cells defining AEC and AER tissues are largely the same, differing only in the magnitude of their signalling centre potential. Hence, AEC does not seem to involve a novel transcriptional programme specific for regenerationcompetent species, but rather the activation of a programme that is highly reminiscent of developmental AER, at least in Xenopus. Moreover, by identifying transcriptomic and morphological differences between the specialized wound epidermis of an amputated tail and limb, we demonstrated that, at the cellular level, appendage regeneration is context dependent and warrants caution for cross-paradigm comparisons. Indeed, it is likely that other regeneration paradigms may use different cell types and transcriptional programmes for their specialised wound epidermis [e.g. zebrafish fin AEC does not express $F g f 8$ (Shibata et al., 2016)]. Nonetheless, amniotes, including humans, have a developmental AER (Kelley and Fallon, 1976). Therefore, our results suggest that mammals have the transcriptional programme to orchestrate limb regeneration, but fail to redeploy the AER cell transcriptional programme upon injury. These results prompted us to characterize regulators of AER cell formation.

Recent research has focused on the intrinsic properties of mesodermal tissue and its ability to induce specialized wound 
epidermis (via Fgf10 expression), supported by the observation that transplantation of mesoderm tissue from regeneration-incompetent limbs prevents regeneration in competent Xenopus limbs (Sessions and Bryant, 1988; Yokoyama et al., 2000). However, this approach is not able to discriminate whether cells are intrinsically incompetent or whether secreted factors cause this effect, as both would be transferred at the same time (as well as the numerous caveats associated with tissue transplantation). Moreover, this hypothesis does not explain why FGF10 is insufficient to induce AER cells across the entire epidermis or why regeneration outcomes are significantly correlated with the extent of ossification at the amputation plane (Dent, 1962; Nye and Cameron, 2005; Wolfe et al., 2000). Inspired by our scRNA-seq data, we sought to determine whether other secreted factors could also be contributing to regeneration incompetency. To our knowledge, there is no practical way to obtain secreted factors from regenerationincompetent tadpoles and transfer them to regeneration-competent animals in vivo. Therefore, we established ex vivo cultures that faithfully recapitulated in vivo regeneration to test this critical hypothesis. We identified AER cells in the ex vivo limbs using spatially resolved and quantitative measurement of epidermal $F g f 8$ via HCR (Choi et al., 2018). Our scRNA-seq data demonstrated that high epithelial $F g f 8$ expression is a unique late-stage marker in the establishment of AER cell identity (Fig. 3D), and therefore Fgf8 positivity in our experimental set-up corresponds with high precision to the AER cell type. By using our explant systems and conducting co-culture and conditioned media experiments, both of which would be inaccessible in vivo, we found that secreted inhibitory factors in regeneration-incompetent tadpoles negatively impact AER cell formation.

To further explore this observation, we surveyed our scRNA-seq data and saw that a number of putative inhibitors (e.g. Noggin) were enriched in chondrogenic cell types, suggesting that factors secreted from the chondrogenic lineage may prevent AER cell formation. To test this hypothesis, we perturbed two genes previously associated with regeneration: Noggin (Beck et al., 2006; Pearl et al., 2008) and Fgf10 (Yokoyama et al., 2001). Previous analysis of these genes was limited to the study of exogenous perturbations and their effect on regeneration outcome, without providing a model involving their endogenous function and their interaction. For example, although NOGGIN overexpression was shown to block regeneration, we show here for the first time that secreted factors in regenerationincompetent tadpoles block AER cell formation and that endogenous NOGGIN is one of the factors causing this effect. Similarly, although FGF10 was shown to restore regeneration competency, it was not known that FGF10 activity operates upstream of chondrogenesis and NOGGIN to influence regeneration-outcome. Altogether, in this work we have systematically assessed which cell types express FgflO and Noggin, how they act on cell types to impact regeneration, and how they operate within our proposed cellular mechanism.

We then tested our model in vivo and found that removal of secreted inhibitors (e.g. NOGGIN) or blocking the source of secreted inhibitors (chondrogenic progression via FGF10 application) could indeed improve the regeneration outcome in regeneration-defective stages. Moreover, we demonstrated that NOGGIN attenuates the positive effect of FGF10 application, further highlighting the downstream role played by the secreted inhibitors. Overall, these results align with previous transplantation experiments showing that mesoderm from regeneration-incompetent limbs is inhibitory to regeneration (Sessions and Bryant, 1988; Yokoyama et al., 2001, 2000). However, in contrast to previous interpretations, we suggest that an important contributor to this phenomenon is the enrichment of chondrogenic cell abundance within the mesoderm tissue that express inhibitory secreted factors.

We further showed that, by manipulating NOGGIN and FGF10 levels, we could improve amputation outcomes in regenerationrestricted and/or -incompetent tadpoles. We saw that anti-NOGGIN beads had a mild effect compared with FGF10 beads (Fig. 5A,G), which may suggest that there are other inhibitors secreted from the chondrogenic lineage (e.g. Chrdll and Frzb) that must also be eliminated to ensure robust regeneration. However, the mild effect of anti-NOGGIN may also be due to technical problems with the perturbation (e.g. limited duration and/or diffusivity of antibody delivery), and that a more-complete inhibition of NOGGIN function would further improve the amputation outcome.

It is well established that a salamander blastema will form only in a location distal to the amputation plane, a phenomenon termed as the rule of distal transformation (Butler, 1955; Nacu and Tanaka, 2011; Stocum, 1981). In our explants, we also detect that only distal sites started to form a blastema (Fig. 3A), aligning with the rule of distal transformation. Interestingly, by manipulating NOGGIN and FGF10, we also could observe AER cell formation at the proximal sites of explants (Fig. 5F). However, it remains unclear whether these proximal AER cells can enable the formation of a proximal blastema. Further work is required to investigate the relationship between the rule of distal transformation and AER cells.

Benefiting from the stage-dependent regeneration competency in Xenopus, our scRNA-seq datasets can discriminate true regeneration responses from injury responses. The majority of limb regeneration-associated genes are derived from salamanders, where an injury control is not necessarily available (as these animals can always regenerate their limbs). We found that many genes associated with salamander limb regeneration (e.g. Dpt and Prdx2) (Gerber et al., 2018; Haas and Whited, 2017; Leigh et al., 2018) are upregulated upon injury in a subset of fibroblasts, regardless of regeneration competency. In a different context, recent single-cell analysis of mouse digit tip amputations suggests that, independent of the regeneration outcome, some fibroblast populations express blastema-associated genes (e.g. Prickle1, Fbn2 and Lrrc17) (Storer et al., 2020). We also see these genes upregulated upon injury in a subset of fibroblasts, but again this response is not specific to regeneration. These results suggest that there may be a conserved response to injury for mesenchymal cells in amphibians and mammals, and may be reflecting early suggestions by Tassava et al. that an injury can induce morphologically assigned 'dedifferentiation' that fails to establish a blastema without a specialized wound epidermis (Tassava and Loyd, 1977; Tassava and Mescher, 1975). Indeed, we observed lower levels of some regeneration-associated distal mesenchyme genes (e.g. Shh) in the subset of fibroblasts when there are no AER cells (Fig. S10), correlating with regeneration competency. Nonetheless, our results are insufficient to determine: (1) whether the fibroblast cells progressively become intrinsically incompetent to fully dedifferentiate; or (2) whether, without signals from signalling centre potent AER cells, they fail to fully dedifferentiate. Moreover, although a subset of fibroblasts can express genes from multiple lineages, the functional consequences of this gain of transcriptional multipotency and how it resolves during varying stages of regeneration competency remain unclear. Further work on injuryinduced mesenchymal plasticity, its interaction with AER cells and cross-species comparison on this topic will be required. Nevertheless, these results underscore that caution is needed when interpreting experiments involving injury (e.g. transplantation), as 


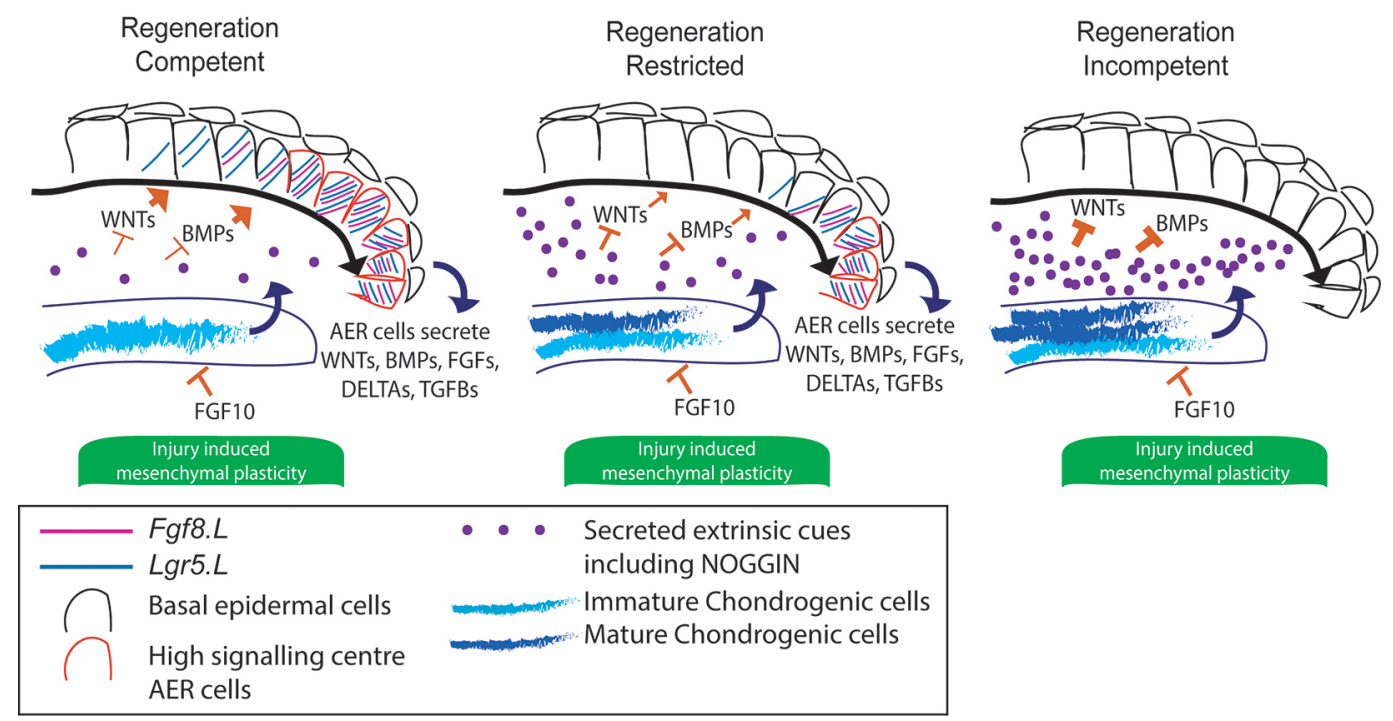

Fig. 6. Schematics describing the proposed model: secreted inhibitory factors associated with chondrogenic progression block AER cell formation. Secreted factors such as WNTs and BMPs support AER cell formation at the amputation plane. During development, chondrogenesis leads to the accumulation of secreted inhibitory factors, including NOGGIN, which results in failure to establish AER cells (Fgf8.L+/Lgr5.S+). FGF10 can suppress chondrogenesis. Amputations, independent of the regeneration outcome, induce injury-induced mesenchymal transcriptional plasticity.

well as the concern that previously implied 'regeneration-genes' may be injury response genes.

Overall, we propose a new cellular model of regeneration incompetency, in which chondrogenesis-associated secreted factors inhibit AER cell formation. Although it remains unclear whether chondrogenesis itself directly inhibits limb regeneration, there are multiple observations from our work and others that support this hypothesis (Dent, 1962; Nye and Cameron, 2005; Wolfe et al., 2000). Our model suggests new avenues for cross-species studies aiming to decipher limb development and regeneration, and can explain why Xenopus limb amputations at proximal versus distal sites exhibit different regeneration outcomes, as proximal sites are associated with more advanced stages of chondrogenesis (Dent, 1962; Nye and Cameron, 2005; Wolfe et al., 2000). Furthermore, the pace of chondrogenesis may have an association with limb regeneration ability across species, such that terrestrial warm-blooded animals may have a more robust and fast-paced chondrogenesis programme compared with regeneration-competent aquatic cold-blooded animals. Indeed, limb regeneration-incompetent species such as chicken or mouse have a faster limb chondrogenesis programme during their development compared with regeneration-competent axolotl and Xenopus. Additionally, although a side-by-side comparative study would be required, mice bone fractures have been documented to heal faster compared with those in axolotl (Hutchison et al., 2007; Vortkamp et al., 1998). It is well established that chondrogenic programmes are heavily influenced by BMP pathway activity. The ratio of BMP agonist/antagonist (e.g. BMP4/ NOGGIN) during development, injury or upon limb amputation may be different between limb regeneration-competent and -incompetent animals. This difference may also be connected to observed Noggin phenotypes across species. Specifically, adding exogenous Noggin results in extended AER maintenance in chicken (Pizette and Niswander, 1999) and mouse (Wang et al., 2004), whereas it abolishes AER in Xenopus (Jones et al., 2013). Targeted comparative studies on these topics will be the subject of future work.

It remains unclear how our identified cellular mechanisms are associated with robust regenerative abilities of some salamanders. Based on current results, regeneration-competent axolotls are suggested to not have a developmental AER (Purushothaman et al., 2019), but can form AEC. Meanwhile AER-associated FGFs are expressed in axolotl mesenchyme (Purushothaman et al., 2019). Hence, it is tempting to speculate that limb regeneration-competent salamanders could withstand inhibitory secreted factors because of the location and, potentially, higher absolute amount of AER cell signals in mesenchymal rather than epidermal cells. Additionally, in contrast to axolotl limb regeneration, where a more homogenous mesenchymal transcriptional response was suggested (Gerber et al., 2018), we identified that only a subset of fibroblast populations can gain transcriptional multipotency and express genes associated with blastema. Whether these differences between species result in more robust regenerative abilities requires further work.

Finally, in this work we have identified a cellular mechanism governing regeneration incompetency in developing tadpoles, although it remains unclear whether similar principles apply in adult frogs with a more definite limb. Manipulation of chondrogenic programmes in adult frogs and other regeneration-incompetent species may lead to novel approaches to promote limb regeneration, albeit with additional barriers to regeneration (e.g. scarring and more complex immune responses) that may have to be overcome. Altogether, our work suggests a new cellular model of limb regeneration (Fig. 6), which unites disparate tissue and gene level findings in the field, and suggests that modulation of secreted factors impacting on epidermal populations has the potential to unlock the ability to regrow lost limbs in non-regenerative higher vertebrates.

\section{MATERIALS AND METHODS}

\section{Tadpole generation and husbandry}

Tadpoles were generated and staged as previously described (Aztekin et al., 2019). After NF stage 45, tadpoles were fed once or twice a day with filamentous blue-green algae (ZM spirulina powder) suspended in water. Wild-type Xenopus laevis were used for experiments unless otherwise stated. Tadpoles classified as regeneration competent were NF stage 52-53, regeneration restricted were NF stage 55-56, and regeneration incompetent were NF stage 58-60. Animal experiments were approved by the University Biomedical Services at the University of Cambridge and complied with 
UK Home Office guidelines and the Animals (Scientific Procedures) Act 1986.

\section{Single-cell dissociation, library preparation and sequencing}

For developmental samples, tadpoles were killed and samples were collected at the aforementioned stages. For amputation/regeneration samples, tadpoles were anaesthetized by incubating them with $0.1 \times \mathrm{MMR}$ $0.002 \%$ MS222 (A0377876, Acros Organics), placed on a wet towel and the right hindlimbs were amputated at the presumptive knee/ankle level for regeneration-competent tadpoles, and at the ankle level for regenerationrestricted or -incompetent tadpoles. Afterwards, the tadpoles were returned to fresh water. At 5 days post amputation (dpa), tadpoles were killed and the newly generated tissues on the amputation plane were collected. Contralateral control samples were also collected from these tadpoles, and intact limb buds or autopods, including ankle, were collected. For each scRNA-seq experiment, tissues were collected from a total of 8-10 tadpoles to reduce variance caused by staging differences. Dissociations were performed on a pool of four limbs in an Eppendorf tube with the following protocol. First, the samples were washed with $\mathrm{Ca}$ - and $\mathrm{Mg}$-free $1 \times \mathrm{MBS}$ (Barth-HEPES saline $10 \times$ stock: $88 \mathrm{mM} \mathrm{NaCl}, 1 \mathrm{mM} \mathrm{KCl}, 2.4 \mathrm{mM}$ $\mathrm{NaHCO}_{3}, 0.82 \mathrm{mM} \mathrm{MgSO}{ }_{4} .7 \mathrm{H}_{2} \mathrm{O}, 0.33 \mathrm{mM} \mathrm{Ca}(\mathrm{NO} 3)_{2} .4 \mathrm{H}_{2} \mathrm{O}, 0.41 \mathrm{mM}$ $\mathrm{CaCl}_{2} \cdot 6 \mathrm{H}_{2} \mathrm{O}$ and $10 \mathrm{mM}$ HEPES with $\sim 3 \mathrm{ml}$ of $10 \mathrm{~N} \mathrm{NaOH}$ added to obtain a $\mathrm{pH}$ of 7.4 to 7.6 ). Samples were then incubated with $1 \times$ trypsin (Sigma, $59427 \mathrm{C}$ ) in Ca- and $\mathrm{Mg}$-free $1 \times \mathrm{MBS}$ with $0.5 \mu \mathrm{M}$ EDTA for $10 \mathrm{~min}$ at room temperature on a bench-top shaker at a speed of $300 \mathrm{rpm}$. Trypsin reaction was diluted with $\mathrm{Ca}$ - and $\mathrm{Mg}$-free $1 \times \mathrm{MBS}$ after $10 \mathrm{~min}$. Physical dispersion was applied (10-15 times up and down trituration with a pipette) to samples before, half way and at the end of trypsinization. Cells were spun down at $250 \mathrm{~g}$ for $5 \mathrm{~min}$, the supernatant was taken out and cells were then resuspended in $1 \times \mathrm{Ca}$ - and $\mathrm{Mg}$-free MBS. Cells were passed through a $35 \mu \mathrm{m}$ diameter cell strainer then stained with $20 \mu \mathrm{M}$ Hoechst 33342 (Sigma, 2261) in 1× Ca- and Mg-free MBS for 10-15 min; Hoechst positive cells were sorted using a Sony SH800s Cell Sorter. scRNA-seq libraries were generated using $10 \mathrm{X}$ Genomics ( $\mathrm{v} 3$ chemistry) and sequenced in pools of two samples per lane on an Illumina Novaseq $6000 \mathrm{SP}$ flow cell, with the following parameters: $28 \mathrm{bp}$ - read 1; 8 bp - i7 index; and $91 \mathrm{bp}$ - read 2, as per standard 10X Genomics recommendations.

\section{scRNA-seq: data processing}

Output files from 10X Genomics were processed using CellRanger v3.0.2, with sequences mapped to the Xenopus laevis 9.1 genome (Xenbase, $\mathrm{ftp}: /$ ftp.xenbase.org/pub/Genomics/JGI/Xenla9.1/Xla.v91.repeatMasked.fa.gz and ftp://ftp.xenbase.org/pub/Genomics/JGI/Xenla9.1/1.8.3.2/XL_9.1_ v1.8.3.2.allTranscripts.gff3.gz). Raw counts were normalized by cell library size, and then converted to TPX (transcripts per $10^{4}$ ). Cell calling was performed using CellRanger with default parameters. We further filtered the data according to library size, discarding cells with a total UMI count in the lowest quartile. The main cell types and transcriptional changes remained unchanged if this cell-filtering step is omitted, although the clustering and visualization appears less robust (Fig. S4).

\section{SCRNA-seq: feature selection}

Highly variable genes (HVGs) were selected for clustering and visualization as described previously (Aztekin et al., 2019) (Fano factor $>65$ th percentile, mean expression $>5$ th percentile and mean expression $<80$ th percentile). Our initial analysis revealed that visualization and clustering were strongly influenced by cell cycle state (Fig. S2). To further refine the set of HVGs, we performed factor analysis with the aim of removing genes significantly associated with the cell cycle. Specifically, non-negative matrix factorization was performed on the cosine normalized, log2-transformed normalized counts matrix, using $\mathrm{k}=30$ components ( $\mathrm{R}$ package $n n l m$ ). Factors were manually annotated according to their expression on the UMAP projection, and by inspection of the highest gene loadings for each factor; two factors corresponded to the cell cycle. To minimize the effect of the cell cycle signature on projection/clustering, we identified genes associated with these cell cycle factors (top $10 \%$ gene loadings for each factor) and removed these from the set of HVGs.

\section{ScRNA-seq: visualization and clustering}

Data were projected onto two dimensions using the UMAP algorithm (Becht et al., 2019), with $\log 2$-transformed HVGs, cosine distance as a similarity measure and parameters $\mathrm{k}=15$, min_dist $=0.2$. Clustering was performed as described previously (Aztekin et al., 2019). Briefly, we constructed a graph using the UMAP function fuzzy_simplicial_set with $\mathrm{k}=10$ nearest neighbours, and then performed graphical clustering using the walktrap algorithm (cluster_walktrap from $\mathrm{R}$ package igraph, with steps $=10)$.

\section{scRNA-seq: gene set enrichment and cell cycle analysis}

Single-cell gene set enrichment scores were calculated with the AUCell $\mathrm{R}$ package (Aibar et al., 2017), using HVGs as the background gene set. Cell cycle phase was inferred using CellCycleScoring (R package Seurat) (Butler et al., 2018).

\section{SCRNA-seq: annotation of cell types}

Cell type annotation was performed by manually comparing cluster-specific gene expression patterns [computed using findMarkers in $\mathrm{R}$ package scran (Lun et al., 2016)] with known cell type markers from the literature. Many clusters could be assigned to a well-characterized functional cell type (e.g. Satellite cell). Other clusters could not be unambiguously identified, but were assigned a broad label together with a numeric identifier (e.g. Blood 1). Finally, a few clusters remain unannotated (e.g. Unknown 1). Dotplots of key marker genes of each cell type are provided in Fig. S5.

\section{ScRNA-seq: gene expression visualization}

Gene expression in individual cells is visualized on the UMAP projection with points coloured according to expression level (log10-transformed). Gene expression across groups of cells (e.g. for different clusters or for different stage tadpoles) is shown using dotplots coloured by mean expression ( $\log 10$-transformed, normalized to group with maximal expression). We can detect alleles from both the large (Gene.L) or short (Gene.S) chromosomes present in the pseudotetraploid Xenopus laevis genome. In some figures, we report expression from both the large and short allele; in others, we report whichever allele has higher expression for brevity. Differentially expressed genes were identified using the findMarkers function (using default parameters, and comparing cells from different conditions); results were then visualized as volcano plots.

\section{Regeneration assay and bead experiments}

Affi-gel blue gel beads (BioRad, 1537301) were incubated with the following proteins overnight at $4^{\circ} \mathrm{C}: 2-3 \mu \mathrm{g}$ rabbit-IGG isotype control antibody (ab37415); $2-3 \mu \mathrm{g}$ anti-NOGGIN antibody (ab16054); 0.1\% BSA; $1 \mu \mathrm{g}$ recombinant human FGF10 (R\&D, 345-FG) in 1-2 $\mu 10.1 \% \mathrm{BSA}$; and 1-1.5 $\mu \mathrm{g}$ recombinant human FGF10 (R\&D, 345-FG) and 2.5-4 $\mu \mathrm{g}$ recombinant human NOGGIN (R\&D, 6057-NG) in 3-4 $\mu 10.1 \%$ BSA. Tadpoles were anaesthetized with $0.002 \%$ MS222, placed on a wet towel, and both right and left hindlimbs were amputated from ankle level in either regeneration-restricted or -incompetent tadpoles. Three or four beads were placed on the amputation plane of the right hindlimb. Left hindlimbs served as an internal control for the experiments. Pushing the bead deep in the tissues at the amputation site was avoided as much as possible, and beads were gently positioned instead. Tadpoles were monitored on a wet towel for 3-5 min then tadpoles that retained the beads were placed in fresh water. Tadpoles were killed in between 18 and 21 dpa to assess the regeneration outcome. The difference in the number of digits or digit-like structures between the right to the left limb was quantified for each tadpole

\section{Whole-mount mRNA visualization and hybridization chain reaction (HCR), with or without a combination of immunofluorescence or histology HCR on whole-limb or tail samples}

HCR was applied as described previously (Choi et al., 2018) with modifications, and materials for HCR were purchased from Molecular Instruments unless otherwise stated. Limb and tail samples were fixed with 
$4 \%$ formaldehyde in $1 \times \mathrm{PBS}$ for $40-60 \mathrm{~min}$, permeabilized in $70 \%$ ethanol in $1 \times \mathrm{PBS}$ for $2-4 \mathrm{~h}$, washed briefly with $1 \times \mathrm{PBS}$ and collected in Eppendorf tubes. These procedures were carried out on a rotator at room temperature. The supernatant was taken out, $500 \mu \mathrm{l}$ wash solution was added, and samples were rotated at room temperature for $5 \mathrm{~min}$. The supernatant was taken out and replaced by 400-500 $\mu 1$ hybridization buffer for a $30 \mathrm{~min}$ incubation at $37^{\circ} \mathrm{C}$. In parallel, the probe solution was prepared by diluting mRNAs targeting probes to $30-40 \mathrm{nM}$ in $200 \mu \mathrm{l}$ hybridization buffer and incubated for $30 \mathrm{~min}$ at $37^{\circ} \mathrm{C}$. The hybridization buffer from samples were taken out and probe solution was placed on samples for a 12-16 h incubation at $37^{\circ} \mathrm{C}$. Subsequently, the samples were washed twice for $20 \mathrm{~min}$ with wash buffer and twice for $30 \mathrm{~min}$ with $5 \times \mathrm{SSC}$ - T at room temperature. To visualize probes, amplification solution was prepared by first heating the fluorophore attached hairpins pairs (h1 and h2 hairpins) that match to the probes to $95^{\circ} \mathrm{C}$ for $90 \mathrm{~s}$. Hairpins were then left in the dark at room temperature for $30 \mathrm{~min}$. Afterwards, final amplification solution was prepared at $40-60 \mathrm{nM} \mathrm{h} 1$ and h2 in $200 \mu \mathrm{l}$ amplification buffer. Samples were first incubated in amplification buffer without hairpins for $10 \mathrm{~min}$, then placed in final amplification solution at room temperature, protected from light, for 12-16 h on a rotator. Samples were washed with $2 \times 20 \mathrm{~min}$ SSC-T. Samples were then stored in $1 \times$ PBS.

\section{Whole-mount HCR samples imaging}

For stereomicroscope or confocal imaging of whole samples, the samples were mounted in $0.6-0.8 \%$ ultra-low gelling temperature agar (Sigma, $\mathrm{A} 5030)$ in $1 \times \mathrm{PBS}$.

\section{Sectioning of samples after HCR}

In the subsequent step of the protocol, the samples were protected from light to preserve the HCR signal. The samples were incubated in $15 \%$ sucrose in $1 \times$ PBS at room temperature for $1 \mathrm{~h}$, then $30 \%$ sucrose in $1 \times \mathrm{PBS}$ at $4^{\circ} \mathrm{C}$ overnight. Samples were then placed in OCT solution and incubated at $-80^{\circ} \mathrm{C}$ overnight. Samples were cryosectioned at $5 \mu \mathrm{m}$, stained with $20 \mu \mathrm{M}$ Hoechst (Sigma, 2261) in $1 \times \mathrm{PBS}$ at room temperature for $10 \mathrm{~min}$ and imaged.

\section{Immunostaining}

After sectioning of HCR stained limb, the samples were processed for immunostaining. Samples were blocked with 50\% Cas-Block (Invitrogen, $008120)$ in $1 \times$ PBS-T (1X PBS +0.1 Tween-100) and incubated for $30 \mathrm{~min}$ at room temperature without rotating. Samples were then incubated with antibodies (listed below) at $4{ }^{\circ} \mathrm{C}$ overnight without rotating. Samples were washed with PBS-T for $2 \times 10$ min, blocked with $50 \%$ Cas-Block in $1 \times$ PBS$\mathrm{T}$ for $30 \mathrm{~min}$, and incubated with secondary antibodies (listed below) for $1 \mathrm{~h}$. All these steps were carried out at room temperature without rotating. Samples were washed with $1 \times$ PBS-T twice for $10 \mathrm{~min}$ and with $1 \times \mathrm{PBS}$ twice for $20 \mathrm{~min}$ at room temperature without rotating. After antibody staining, samples were stained with Hoechst and washed once for $5 \mathrm{~min}$ in $1 \times$ PBS at room temperature without rotating. Samples were mounted in $80 \%$ glycerol in $1 \times$ PBS with a coverslip and imaged.

Tail whole-mount HCR staining can be combined with whole-mount immunofluorescence by following the above immunofluorescence protocol, except that the mounting of whole tails were carried out in ultra-low gelling temperature agar for imaging.

\section{HCR probes and hairpins}

Probes for Fgf8.L, Dpt.L, Htra3.L, Prrx1.L and Sp9.L were purchased from Molecular Instruments. Probes were designed against the full-length Xenopus Lgr 5.S, Msx I.L and Fgf10.L mRNA sequence as described previously (Choi et al., 2014). HCR hairpins were purchased from Molecular Instruments.

\section{Primary antibodies and working dilutions}

Primary antibodies and working dilutions were as follows: TP63 [4A4] (Abcam, ab735, 1:200), B-CATENIN (Abcam, ab6302, 1:2000), E-CADHERIN (5D3, DSHB, 1:10), ITGB1 (8C8, DSHB, 1:10) and antiEGFP (Abcam, ab13970, 1:500).

\section{Secondary antibodies}

Secondary antibodies were as follows: goat anti-chicken $\operatorname{IgY}(\mathrm{H}+\mathrm{L})$ secondary antibody, Alexa Fluor 488 (Invitrogen, A11039, 1:500), goat anti-mouse $\operatorname{IgG}(\mathrm{H}+\mathrm{L})$ cross-adsorbed ReadyProbes secondary antibody, Alexa Fluor 594 (Invitrogen, R37121, 1:500) and goat anti-mouse IgG $(\mathrm{H}+\mathrm{L})$ cross-adsorbed ReadyProbes secondary antibody, Alexa Fluor 488 (Invitrogen, R37120, 1:500).

A Leica SP8 upright confocal microscope with a $40 \times / 1.3$ HC PL Apo CS2 Oil objective was used for all confocal images except for Fig. S9B,C, which were taken with a Leica SP8 inverted confocal microscope with a $20 \times / 0.75$ HC PL Apo CS2 Multi. LAS X was used for setting tiled images and a $20 \%$ overlap between tiles was used. Limb whole-mount HCR images were taken via a Leica stereomicroscope equipped with a DFC7000T camera. Fiji was used for maximum projection of $z$-stacks and to adjust contrast to highlight biological relevance. If needed, images were cropped, flipped and/or rotated to highlight biological relevance.

Histological staining can be carried out on cryosectioned HCR samples. Briefly, samples were stained with Hematoxylin and Eosin according to manufacturer's protocol (Abcam, ab245880) then samples were stained for Alcian Blue (Sigma, B8438) according to manufacturer's protocol. Histology images were captured on a Zeiss AxioImager compound microscope.

\section{Ex vivo limb culture method to assess AER cell formation and proximal chondrogenesis}

Limbs were first amputated from presumptive knee/ankle level for regeneration-competent and from ankle level for regeneration-restricted or -incompetent tadpoles. The distal parts of these amputated explants were then removed and the remaining proximal segment was placed in 1000, 500 or $200 \mu \mathrm{l}$ explant media [L-15 (ThermoFisher Scientific, 21083027), $1 \times$ Antibiotic-Antimycotic (ThermoFisher Scientific, 15240062) and 20\% Fetal Bovine Serum Superior (Sigma, S0615)] in 12, 24 or 96-well plates, respectively. Explants were cultured for 3 days without changing the media. After 3 days, to quantify AER cell formation, the explants were fixed and proceeded to the HCR protocol; to quantify proximal chondrogenesis, the explants were fixed with $4 \%$ formaldehyde, mounted in $0.6 \%$ Low-Melt agar and directly imaged by stereomicroscopy. Explants emit autofluorescence. Although the abundant HCR signal can be seen despite the autofluorescence, to discriminate the HCR signal from autofluorescence in finer detail, sample images were taken in red and green channel separately with the same exposure and gain settings, and then merged in Fiji. In merged images, the background signal due to autofluorescence was visualized as yellow and the HCR signal was either red or green. As AER cells were largely detected as a monolayer population, AER cell formation was calculated by measuring the length of the $F g f 8 . L$ signal on the amputation plane using Fiji segmented line option. The proximal chondrogenesis can be visually distinguished and, to determine the chondrogenesis length, chondrogenic structure length from top to bottom was also measured using Fiji. Samples where a clear chondrogenesis was not visible were omitted from further analysis. These images were taken in bright field and measurements were carried out in Fiji.

For drug and recombinant protein treatments, the explants were placed in culture media containing the following small molecules concentration or recombinant protein amounts, unless otherwise stated: $100 \mu \mathrm{M}$ ICRT3 (Sigma, SML0211), $100 \mu \mathrm{M}$ SU-5402 (Sigma, SML0443), $50 \mu \mathrm{M}$ SB505124 (Sigma, S4696), $100 \mu \mathrm{M}$ DAPT (Sigma, D5942), $2.5 \mu \mathrm{M}$ LDN193189 (Stemgent, 04-0074), 500 ng human recombinant FGF10 (R\&D, 345-FG), $1.25 \mu \mathrm{g}$ human recombinant NOGGIN (R\&D, 6057-NG) and $500 \mathrm{ng}$ human recombinant BMP4 (R\&D, 314-BP). Drugs were prepared in DMSO, and recombinant proteins were prepared in $0.1 \%$ BSA. Smallmolecule experiments were conducted in 24-well plates. Recombinant protein experiments were carried out in 96-well plates. A maximum of six explants were placed in 24-well plates. One explant was put in one well of 96-well plate for recombinant protein treatments. In all chemical and recombinant protein perturbation experiments, one limb of the same animal was subjected to the perturbation, and the contralateral limb served as a control. These control explants were exposed to solution containing matching DMSO or BSA concentration in $1 \times \mathrm{PBS}$ for chemical or 
recombinant protein perturbations, respectively. Perturbation and control samples were pooled separately at the end of the experiments and stained.

\section{EdU labelling}

Ex vivo limbs were cultured with $10 \mu \mathrm{M}$ EdU (ThermoFisher Scientific, C10337) for 3 days in dark foiled cover. Afterwards, samples were fixed and Fgf8.L mRNA was stained using the HCR protocol, followed by cryosectioning, as described above. Sections were subjected to the ClickIt reaction, as described in the manufacturer's protocol (ThermoFisher Scientific, C10337). Hoechst was added at the end of the protocol. Samples were visualized by confocal microscopy as described above. Fgf8.L-positive cells, and both EdU- and $F g f 8 . L$-positive cells on the amputation plane were manually counted, and the percentage of EdU-positive Fgf8.L-positive cells were calculated for each sample.

\section{Bead experiment for proximal chondrogenesis}

Beads were prepared as described above. Explants from regenerationrestricted tadpoles were harvested as described above and beads were implanted on the proximal site of explants. At $3 \mathrm{dpa}$, explants that no longer contained a bead at their proximal site (presumably due to repulsion) were omitted from further analysis. At $3 \mathrm{dpa}$, samples were imaged without fixation and the extent of chondrogenesis was measured by Fiji

\section{DiO labelling}

$\mathrm{DiO}\left[\mathrm{DiO}^{\prime} ; \mathrm{DiOC}_{18}(3)\right.$ (3,3'-dioctadecyloxacarbocyanine perchlorate), ThermoFisher Scientific, D275] was prepared by dipping a tip in the DiO-containing powder tube and placing the tip in a $10 \mu 1$ of $96-100 \%$ ethanol in an Eppendorf tube. A glass needle tip was then dipped in the diluted $\mathrm{DiO}$ solution and harvested ex vivo limbs were labelled on a wet towel. These cultures were placed in ex vivo culture media and explants were imaged every day with a stereomicroscope.

\section{Ex vivo limb co-culture, and conditioned media experiments}

For co-culture experiments, one regeneration-competent and one regeneration-incompetent limb explant were incubated together in $200 \mu \mathrm{l}$ explant media in one well of 96-well plate. For antibody experiments, one limb of each animal served as a control and was incubated with $1 \mu \mathrm{g}$ rabbitIGG isotype control antibody (ab37415), while the contralateral limb was incubated with $1 \mu \mathrm{g}$ anti-NOGGIN antibody (ab16054). Antibodies and media were added only at the beginning of the cultures and were not replaced during the experiment.

For conditioned media experiments, conditioned media supplying and receiving explants were prepared separately. Supplying explants were prepared 1 day before harvesting receiving explants and incubated in $200 \mu 1$ explant media in one well of 96-well plate. After 1 day, media from the supplying explant was collected and used to culture the newly harvested receiving explant, and fresh media were added for supplying explant. This change of media procedure was repeated for 3 days. For antibody experiments, supplying explant media was collected and pre-incubated with $1 \mu \mathrm{g}$ antibodies for 25-30 $\mathrm{min}$ at room temperature on a rotator, then the pre-incubated media was placed on the receiving explants.

\section{Replicate information and statistical tests}

Sample sizes were not pre-determined in any experimental setup. In this work, biological replicates refer to samples obtained from multiple animal batches and to experiments carried out on different days. In all experiments, the number of independent tadpole limbs assayed is recorded and denoted by $n$ in the text and figure legends. In all experiments, wild-type tadpoles were used from tanks that contain multiple batches (tadpoles raised from different father and/or mother). In all explant perturbation experiments, samples were compared with their contralateral controls and a MannWhitney $U$-test was used to determine statistical significance. For regeneration and bead experiments, a $t$-test was used. To assess the significance of proximal $\mathrm{Fg} f 8$ expression in explants (Fig. 5F), Fisher's exact test was used.

\section{Acknowledgements}

We thank Katarzyna Kania and the Cambridge Institute Genomics Core for their support with this work on the 10X-Genomics and sequencing library preparations. The transgenic testes used in this study were obtained from the European Xenopus Resource Centre, curated with funding from the Wellcome Trust/BBSRC, and maintained by the University of Portsmouth, School of Biological Sciences. We thank R. Jones-Green for excellent animal care. We thank H. Ma for use of her stereomicroscope, and E. Rawlins for use of her Zeiss Axiolmager compound microscope. We thank B. Steventon, E. Rawlins, and members of the Marioni and Simons labs for general discussion about the project. We thank R. Butler for assistance in image analysis.

\section{Competing interests}

The authors declare no competing or financial interests.

\section{Author contributions}

Conceptualization: C.A.; Methodology: C.A., T.W.H.; Software: T.W.H.; Validation: C.A., T.W.H.; Formal analysis: T.W.H.; Investigation: C.A.; Resources: J.M., J.G.; Data curation: C.A., T.W.H.; Writing - original draft: J.J., C.A.; Writing - review \& editing: B.D.S., J.J., J.M., C.A., T.W.H.; Supervision: B.D.S., J.J., J.M., J.G.; Project administration: C.A.; Funding acquisition: B.D.S., J.J., J.M., J.G., C.A.

\section{Funding}

C.A. is funded by the University of Cambridge and the Cambridge Trust. J.J. and J.G. are funded by a grant from the Wellcome Trust (101050/Z/13/Z). T.W.H., J.M and B.D.S. are funded as part of a Wellcome Trust Strategic Award to study cell fate decisions (105031/D/14/Z). T.W.H. is also supported by a European Molecular Biology Organization Long-Term Fellowship (ALTF 606-2018). B.D.S. also acknowledges funding from the Royal Society E.P. Abraham Research Professorship (RPIR1/180165) and the Wellcome Trust (098357/Z/12/Z). J.M. acknowledges core funding from the European Molecular Biology Laboratory and Cancer Research UK (A17197). This work is funded by grants from the Wellcome Trust (101050/Z/13/Z) and the Medical Research Council (MR/P000479/1), and was supported by a Gurdon Institute core grant from Cancer Research UK (C6946/ A14492) and the Wellcome Trust (092096/Z/10/Z). Open access funding provided by University of Cambridge. Deposited in PMC for immediate release.

\section{Data availability}

Code is available at https://github.com/MarioniLab/

XenopusLimbRegeneration2020. Sequencing data, together with processed counts matrices, are available on ArrayExpress under the accession number E-MTAB-9104. We provide an interactive online tool to explore our dataset at https://marionilab.cruk.cam.ac.uk/XenopusLimbRegeneration/.

\section{Peer review history}

The peer review history is available online at https://journals.biologists.com/dev/ article-lookup/doi/10.1242/dev.199158

\section{References}

Aibar, S., González-Blas, C. B., Moerman, T., Huynh-Thu, V. A., Imrichova, H., Hulselmans, G., Rambow, F., Marine, J.-C., Geurts, P., Aerts, J. et al. (2017). SCENIC: single-cell regulatory network inference and clustering. Nat. Methods 14, 1083-1086. doi:10.1038/nmeth.4463

Aztekin, C., Hiscock, T. W., Marioni, J. C., Gurdon, J. B., Simons, B. D. and Jullien, J. (2019). Identification of a regeneration-organizing cell in the Xenopus tail. Science 364, 653-658. doi:10.1126/science.aav9996

Becht, E., Mclnnes, L., Healy, J., Dutertre, C.-A., Kwok, I. W. H., Ng, L. G. Ginhoux, F. and Newell, E. W. (2019). Dimensionality reduction for visualizing single-cell data using UMAP. Nat. Biotechnol. 37, 38-44. doi:10. $1038 /$ nbt.4314

Beck, C. W., Christen, B., Barker, D. and Slack, J. M. W. (2006). Temporal requirement for bone morphogenetic proteins in regeneration of the tail and limb of Xenopus tadpoles. Mech. Dev. 123, 674-688. doi:10.1016/j.mod.2006. 07.001

Beck, C. W., Izpisúa Belmonte, J. C. and Christen, B. (2009). Beyond early development: Xenopus as an emerging model for the study of regenerative mechanisms. Dev. Dyn. 238, 1226-1248. doi:10.1002/dvdy.21890

Boehm, B., Westerberg, H., Lesnicar-Pucko, G., Raja, S., Rautschka, M., Cotterell, J., Swoger, J. and Sharpe, J. (2010). The role of spatially controlled cell proliferation in limb bud morphogenesis. PLoS Biol. 8, e1000420. doi:10. 1371/journal.pbio. 1000420

Butler, E. G. (1955). Regeneration of the urodele forelimb after reversal of its proximo-distal axis. J. Morphol. 96, 265-281. doi:10.1002/jmor.1050960204

Butler, A., Hoffman, P., Smibert, P., Papalexi, E. and Satija, R. (2018). Integrating single-cell transcriptomic data across different conditions, technologies, and species. Nat. Biotechnol. 36, 411-420. doi:10.1038/nbt.4096 
Campbell, L. J. and Crews, C. M. (2008). Molecular and cellular basis of regeneration and tissue repair: wound epidermis formation and function in urodele amphibian limb regeneration. Cell. Mol. Life Sci. 65, 73-79. doi:10.1007/s00018007-7433-z

Campbell, L. J., Suárez-Castillo, E. C., Ortiz-Zuazaga, H., Knapp, D., Tanaka E. M. and Crews, C. M. (2011). Gene expression profile of the regeneration epithelium during axolotl limb regeneration. Dev. Dyn. 240, 1826-1840. doi:10. 1002/dvdy.22669

Cannata, S. M., Bernardini, S. and Filoni, S. (1992). Regenerative responses in cultured hindlimb stumps of larvalXenopus laevis. J. Exp. Zool. 262, 446-453. doi:10.1002/jez.1402620412

Choi, H. M. T., Beck, V. A. and Pierce, N. A. (2014). Next-generation in situ hybridization chain reaction: higher gain, lower cost, greater durability. ACS Nano 8, 4284-4294. doi:10.1021/nn405717p

Choi, H. M. T., Schwarzkopf, M., Fornace, M. E., Acharya, A., Artavanis, G. Stegmaier, J., Cunha, A. and Pierce, N. A. (2018). Third-generation in situ hybridization chain reaction: multiplexed, quantitative, sensitive, versatile, robust Development 145, dev165753. doi:10.1242/dev.165753

Christen, B. and Slack, J. M. W. (1997). FGF-8ls associated with anteroposterior patterning and limb regeneration in Xenopus. Dev. Biol. 192, 455-466. doi:10. 1006/dbio.1997.8732

Christensen, R. N. and Tassava, R. A. (2000). Apical epithelial cap morphology and fibronectin gene expression in regenerating axolotl limbs. Dev. Dyn. 217 216-224. doi:10.1002/(SICl)1097-0177(200002)217:2<216::AID-DVDY8>3.0. $\mathrm{CO} ; 2-8$

Dent, J. N. (1962). Limb regeneration in larvae and metamorphosing individuals of the South African clawed toad. J. Morphol. 110, 61-77. doi:10.1002/jmor 1051100105

D'Jamoos, C. A., McMahon, G. and Tsonis, P. A. (1998). Fibroblast growth factor receptors regulate the ability for hindlimb regeneration in Xenopus laevis. Wound Repair. Regen. 6, S-388-S-397. doi:10.1046/j.1460-9568.1998.60415.x

Gerber, T., Murawala, P., Knapp, D., Masselink, W., Schuez, M., Hermann, S., Gac-Santel, M., Nowoshilow, S., Kageyama, J., Khattak, S. et al. (2018) Single-cell analysis uncovers convergence of cell identities during axolotl limb regeneration. Science 362, eaaq0681. doi:10.1126/science.aaq0681

Ghosh, S., Roy, S., Séguin, C., Bryant, S. V. and Gardiner, D. M. (2008). Analysis of the expression and function of Wnt-5a and Wnt-5b in developing and regenerating axolotl (Ambystoma mexicanum) limbs. Dev. Growth Differ. 50 289-297. doi:10.1111/j.1440-169X.2008.01000.x

Haas, B. J. and Whited, J. L. (2017). Advances in Decoding Axolotl Limb Regeneration. Trends Genet. 33, 553-565. doi:10.1016/j.tig.2017.05.006

Han, M.-J., An, J.-Y. and Kim, W.-S. (2001). Expression patterns of Fgf-8 during development and limb regeneration of the axolotl. Dev. Dyn. 220, 40-48. doi:10. 1002/1097-0177(2000)9999:9999<::AID-DVDY1085>3.0.CO;2-8

Hay, E. D. and Fischman, D. A. (1961). Origin of the blastema in regenerating limbs of the newt Triturus viridescens: an autoradiographic study using tritiated thymidine to follow cell proliferation and migration. Dev. Biol. 3, 26-59. doi:10. 1016/0012-1606(61)90009-4

Hutchison, C., Pilote, M. and Roy, S. (2007). The axolotl limb: a model for bone development, regeneration and fracture healing. Bone 40, 45-56. doi:10.1016/j. bone.2006.07.005

Jones, T. E. M., Day, R. C. and Beck, C. W. (2013). Attenuation of bone morphogenetic protein signaling during amphibian limb development results in the generation of stage-specific defects. J. Anat. 223, 474-488. doi:10.1111/joa. 12098

Kato, T., Miyazaki, K., Shimizu-Nishikawa, K., Koshiba, K., Obara, M., Mishima, H. K. and Yoshizato, K. (2003). Unique expression patterns of matrix metalloproteinases in regenerating newt limbs. Dev. Dyn. 226, 366-376. doi:10. 1002/dvdy.10247

Kelley, R. O. and Fallon, J. F. (1976). Ultrastructural analysis of the apical ectodermal ridge during vertebrate limb morphogenesis: I. The human forelimb with special reference to gap junctions. Dev. Biol. 51, 241-256. doi:10.1016/00121606(76)90141-X

Knapp, D., Schulz, H., Rascon, C. A., Volkmer, M., Scholz, J., Nacu, E., Le, M. Novozhilov, S., Tazaki, A., Protze, S. et al. (2013). Comparative transcriptional profiling of the axolotl limb identifies a tripartite regeneration-specific gene program. PLoS ONE 8, e61352. doi:10.1371/journal.pone.0061352

Leigh, N. D., Dunlap, G. S., Johnson, K., Mariano, R., Oshiro, R., Wong, A. Y., Bryant, D. M., Miller, B. M., Ratner, A., Chen, A. et al. (2018). Transcriptomic landscape of the blastema niche in regenerating adult axolotl limbs at single-cell resolution. Nat. Commun. 9, 5153. doi:10.1038/s41467-018-07604-0

Li, H., Wei, X., Zhou, L., Zhang, W., Wang, C., Guo, Y., Li, D., Chen, J., Liu, T., Zhang, Y. et al. (2020). Dynamic cell transition and immune response landscapes of axolotl limb regeneration revealed by single-cell analysis. Protein Cell 12 57-66. doi:10.1007/s13238-020-00763-1

Lun, A. TL., McCarthy, D. J. and Marioni, J. C. (2016). A step-by-step workflow for low-level analysis of single-cell RNA-seq data with Bioconductor [version 2; peer review: 3 approved, 2 approved with reservations]. F1000Research 5, 2122 doi:10.12688/f1000research.9501.2
Mescher, A. L. (1976). Effects on adult newt limb regeneration of partial and complete skin flaps over the amputation surface. J. Exp. Zool. 195, 117-127. doi:10.1002/jez.1401950111

Miyazaki, K., Uchiyama, K., Imokawa, Y. and Yoshizato, K. (1996). Cloning and characterization of cDNAs for matrix metalloproteinases of regenerating new limbs. Proc. Natl. Acad. Sci. USA 93, 6819-6824. doi:10.1073/pnas.93.13.6819

Monaghan, J. R., Athippozhy, A., Seifert, A. W., Putta, S., Stromberg, A. J., Maden, M., Gardiner, D. M. and Voss, S. R. (2012). Gene expression patterns specific to the regenerating limb of the Mexican axolotl. Biol. Open 1, 937-948. doi:10.1242/bio.20121594

Nacu, E. and Tanaka, E. M. (2011). Limb regeneration: a new development? Annu. Rev. Cell Dev. Biol. 27, 409-440. doi:10.1146/annurev-cellbio-092910-154115

Nacu, E., Gromberg, E., Oliveira, C. R., Drechsel, D. and Tanaka, E. M. (2016) FGF8 and SHH substitute for anterior-posterior tissue interactions to induce limb regeneration. Nature 533, 407-410. doi:10.1038/nature17972

Nieuwkoop, P. D. and Faber, J. (1994). Normal Table of Xenopus Laevis (Daudin) Oxford, UK: Taylor and Francis.

Nye, H. L. D. and Cameron, J. A. (2005). Strategies to reduce variation in Xenopus regeneration studies. Dev. Dyn. 234, 151-158. doi:10.1002/dvdy.20508

Pajni-Underwood, S., Wilson, C. P., Elder, C., Mishina, Y. and Lewandoski, M. (2007). BMP signals control limb bud interdigital programmed cell death by regulating FGF signaling. Development 134, 2359-2368. doi:10.1242/dev.001677

Pearl, E. J., Barker, D., Day, R. C. and Beck, C. W. (2008). Identification of genes associated with regenerative success of Xenopus laevis hindlimbs. BMC Dev. Biol. 8, 66. doi:10.1186/1471-213X-8-66

Pizette, S. and Niswander, L. (1999). BMPs negatively regulate structure and function of the limb apical ectodermal ridge. Development 126, 883-894. doi:10. 1242/dev.126.5.883

Pizette, S., Abate-Shen, C. and Niswander, L. (2001). BMP links limb growth and dorsoventral patterning. Development 128, 4463-4474. doi:10.1242/dev.128.22. 4463

Purushothaman, S., Elewa, A. and Seifert, A. W. (2019). Fgf-signaling is compartmentalized within the mesenchyme and controls proliferation during salamander limb development. eLife 8, e48507. doi:10.7554/eLife.48507

Qin, T., Fan, C.-M., Wang, T.-Z., Sun, H., Zhao, Y.-Y., Yan, R.-J., Yang, L., Shen, W.-L., Lin, J.-X., Bunpetch, V. et al. (2020). Single-cell RNA-seq reveals nove mitochondria-related musculoskeletal cell populations during adult axolotl limb regeneration process. Cell Death Differ. 28, 1110-1125. doi:10.1038/s41418-02000640-8

Rodgers, A. K., Smith, J. J. and Voss, S. R. (2020). Identification of immune and non-immune cells in regenerating axolotl limbs by single-cell sequencing. Exp. Cell Res. 394, 112149. doi:10.1016/j.yexcr.2020.112149

Sessions, S. K. and Bryant, S. V. (1988). Evidence that regenerative ability is an intrinsic property of limb cells inXenopus. J. Exp. Zool. 247, 39-44. doi:10.1002 jez.1402470106

Shibata, E., Yokota, Y., Horita, N., Kudo, A., Abe, G., Kawakami, K. and Kawakami, A. (2016). Fgf signalling controls diverse aspects of fin regeneration. Development 143, 2920-2929. doi:10.1242/dev.140699

Stocum, D. L. (1981). Distal transformation in regenerating double anterior axolot limbs. Development 65, 3-18. doi:10.1242/dev 65.Supplement 3

Storer, M., Mas, A., Robert-Moreno, A., Pecoraro, M., Ortells, M. C., Di Giacomo, V., Yosef, R., Pilpel, N., Krizhanovsky, V., Sharpe, J. et al. (2013). Senescence is a developmental mechanism that contributes to embryonic growth and patterning. Cell 155, 1119-1130. doi:10.1016/j.cell.2013.10.041

Storer, M. A., Mahmud, N., Karamboulas, K., Borrett, M. J., Yuzwa, S. A., Gont A., Androschuk, A., Sefton, M. V., Kaplan, D. R. and Miller, F. D. (2020). Acquisition of a unique mesenchymal precursor-like blastema state underlies successful adult mammalian digit tip regeneration. Dev. Cell 52, 509-524.e9. doi:10.1016/j.devcel.2019.12.004

Tassava, R. A. and Loyd, R. M. (1977). Injury requirement for initiation of regeneration of newt limbs which have whole skin grafts. Nature 268, 49-50. doi:10.1038/268049a0

Tassava, R. A. and Mescher, A. L. (1975). The roles of injury, nerves, and the wound epidermis during the initiation of amphibian limb regeneration. Differentiation 4, 23-24. doi:10.1111/j.1432-0436.1975.tb01439.x

Tassava, R. A. and Olsen, C. L. (1982). Higher vertebrates do not regenerate digits and legs because the wound epidermis is not functional. A hypothesis. Differentiation 22, 151-155. doi:10.1111/j.1432-0436.1982.tb01242.x

Thornton, C. S. (1960). Influence of an eccentric epidermal cap on limb regeneration in Amblystoma larvae. Dev. Biol. 2, 551-569. doi:10.1016/0012 1606(60)90054-3

Thornton, C. S. and Thornton, M. T. (1965). The regeneration of accessory limb parts following epidermal cap transplantation in urodeles. Experientia 21 146-148. doi:10.1007/BF02141984

Tsai, S. L., Baselga-Garriga, C. and Melton, D. A. (2019). Blastemal progenitors modulate immune signaling during early limb regeneration. Development 146 dev169128, doi:10.1242/dev.169128

Tsai, S. L., Baselga-Garriga, C. and Melton, D. A. (2020). Midkine is a dual regulator of wound epidermis development and inflammation during the initiation of limb regeneration. eLife 9, e50765. doi:10.7554/eLife.50765 
Verheyden, J. M. and Sun, X. (2008). An Fgf/Gremlin inhibitory feedback loop triggers termination of limb bud outgrowth. Nature 454, 638-641. doi:10.1038/ nature 07085

Vincent, E., Villiard, E., Sader, F., Dhakal, S., Kwok, B. H. and Roy, S. (2020). BMP signaling is essential for sustaining proximo-distal progression in regenerating axolotl limbs. Development 147, dev170829. doi:10.1242/dev. 170829

Vortkamp, A., Pathi, S., Peretti, G. M., Caruso, E. M., Zaleske, D. J. and Tabin, C. J. (1998). Recapitulation of signals regulating embryonic bone formation during postnatal growth and in fracture repair. Mech. Dev. 71, 65-76. doi:10.1016/S09254773(97)00203-7

Wang, C.-K. L., Omi, M., Ferrari, D., Cheng, H.-C., Lizarraga, G., Chin, H.-J., Upholt, W. B., Dealy, C. N. and Kosher, R. A. (2004). Function of BMPs in the apical ectoderm of the developing mouse limb. Dev. Biol. 269, 109-122. doi:10. 1016/j.ydbio.2004.01.016

Wolfe, A. D., Nye, H. L. D. and Cameron, J. A. (2000). Extent of ossification at the amputation plane is correlated with the decline of blastema formation and regeneration in Xenopus laevis hindlimbs. Dev. Dyn. 218, 681-697. doi:10.1002/ 1097-0177(2000)9999:9999<::AID-DVDY1018>3.0.CO;2-6

Yang, E. V., Gardiner, D. M., Carlson, M. R. J., Nugas, C. A. and Bryant, S. V. (1999). Expression of Mmp-9 and related matrix metalloproteinase genes during axolotl limb regeneration. Dev. Dyn. 216, 2-9. doi:10.1002/(SICl)10970177(199909)216:1<2::AID-DVDY2>3.0.CO;2-P

Yokoyama, H., Yonei-Tamura, S., Endo, T., Izpisúa Belmonte, J. C., Tamura, K. and Ide, H. (2000). Mesenchyme with fgf-10 expression is responsible for regenerative capacity in Xenopus limb buds. Dev. Biol. 219, 18-29. doi:10.1006/ dbio.1999.9587

Yokoyama, H., Ide, H. and Tamura, K. (2001). FGF-10 stimulates limb regeneration ability in Xenopus laevis. Dev. Biol. 233, 72-79. doi:10.1006/dbio.2001.0180

Yokoyama, H., Maruoka, T., Ochi, H., Aruga, A., Ohgo, S., Ogino, H. and Tamura, K. (2011). Different requirement for $W n t / \beta$-catenin signaling in limb regeneration of larval and adult Xenopus. PLOS ONE 6, e21721. doi:10.1371/ journal.pone.0021721 


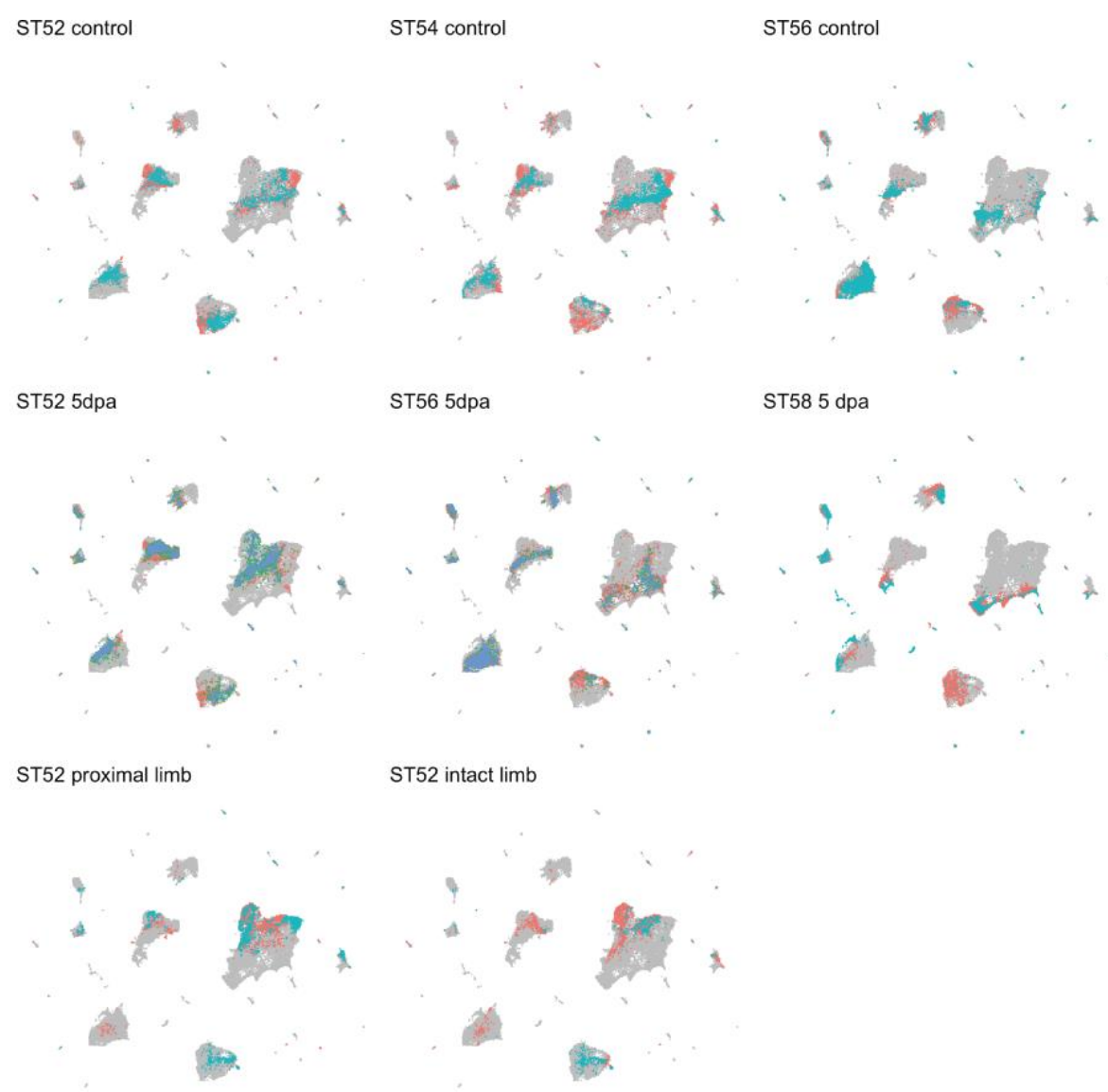

Figure S1: Contribution of different conditions to the pooled UMAP projection.

UMAP visualization of cells from all conditions and replicates, allowing the identification of transcriptional changes that are consistent across replicates. Grey dots: cells from all samples; red, blue, green dots: cells from different biological replicates for the selected sample. 


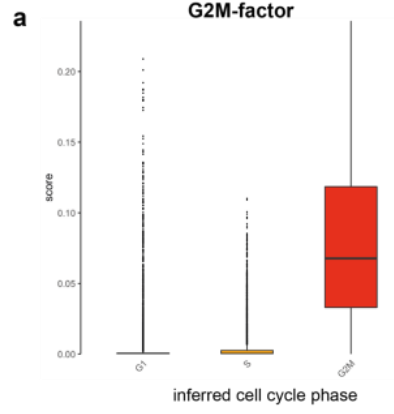

b

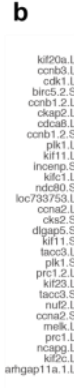

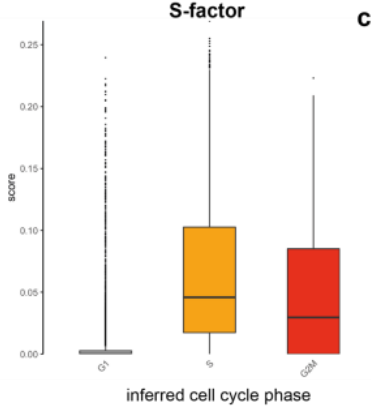

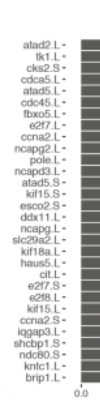

S-factor

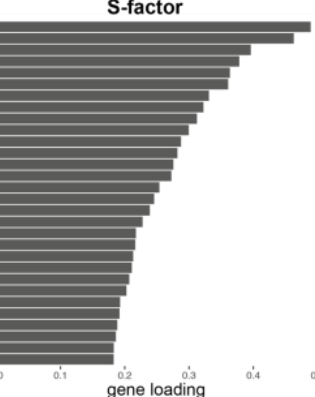

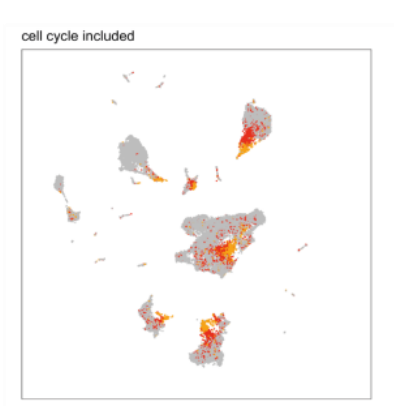

d
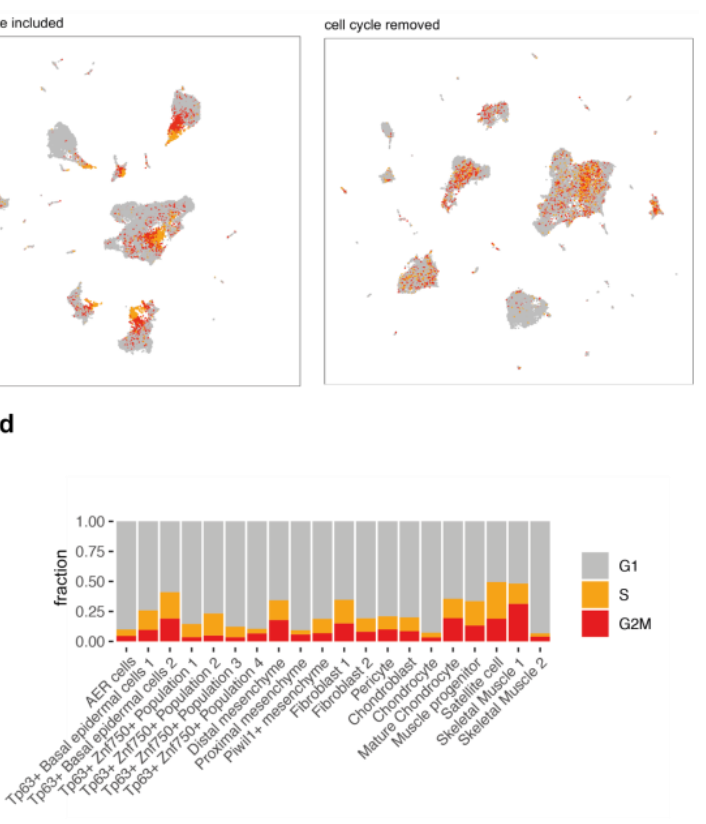

\section{Figure S2: Detection and removal of the cell cycle signature.}

(a) Unbiased factor analysis identified two factors that correspond to computationally-inferred cell cycle phases (G2M-factor, left; S-factor, right). (b) Factor loadings for the top 30 genes associated with cell cycle factors. (c) Removal of genes with high loadings for either G2M- or S-factors significantly reduces the influence of cell cycle phase on the UMAP projection. Dot color indicates inferred cell cycle phase. (d) Inferred cell cycle states for selected cell types. 


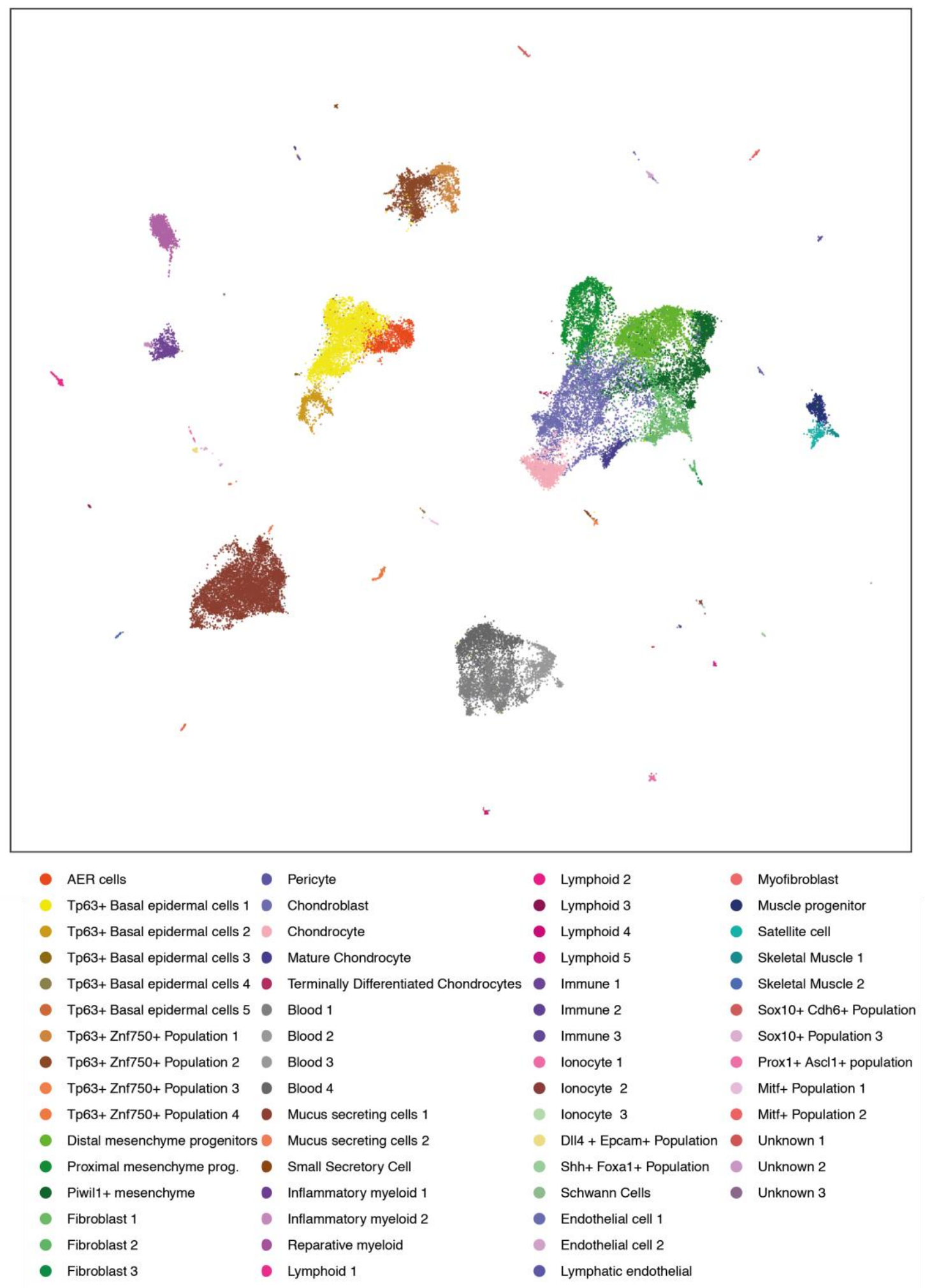

Figure S3: An atlas of cell types in developing and amputated limbs at different stages of regeneration-competence.

Pooled UMAP visualization of Xenopus limb cells, with colors representing distinct cluster identities. 


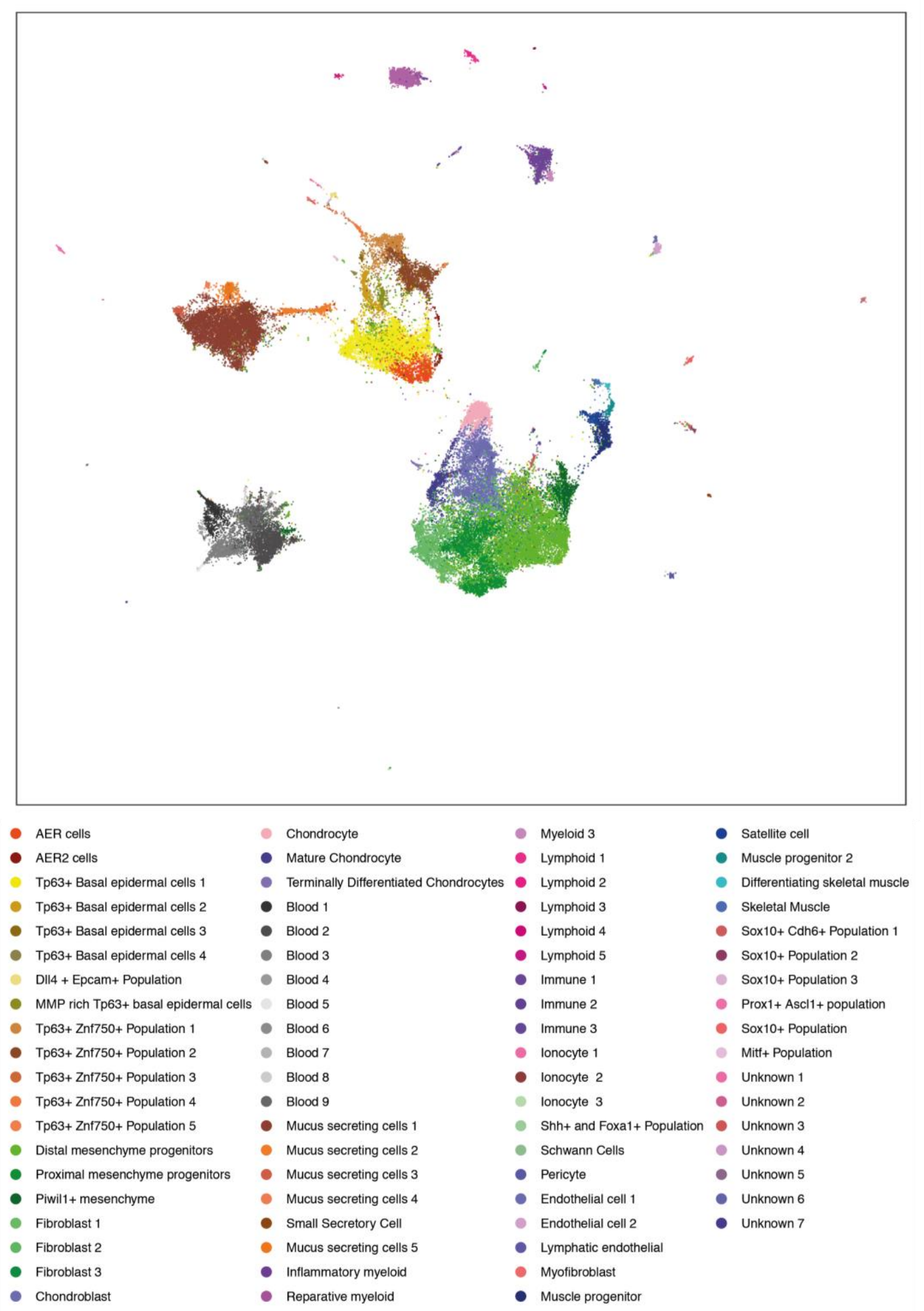

Figure S4: An expanded atlas of the Xenopus limb using less stringent cell filtering protocols.

Pooled UMAP visualization and clustering of all barcodes that are identified as cells using cellRanger with default parameters. The majority of transcriptional states are similar to Figure S3, although a fraction of low-UMI mesenchymal cells appear mislocalized across the atlas. 

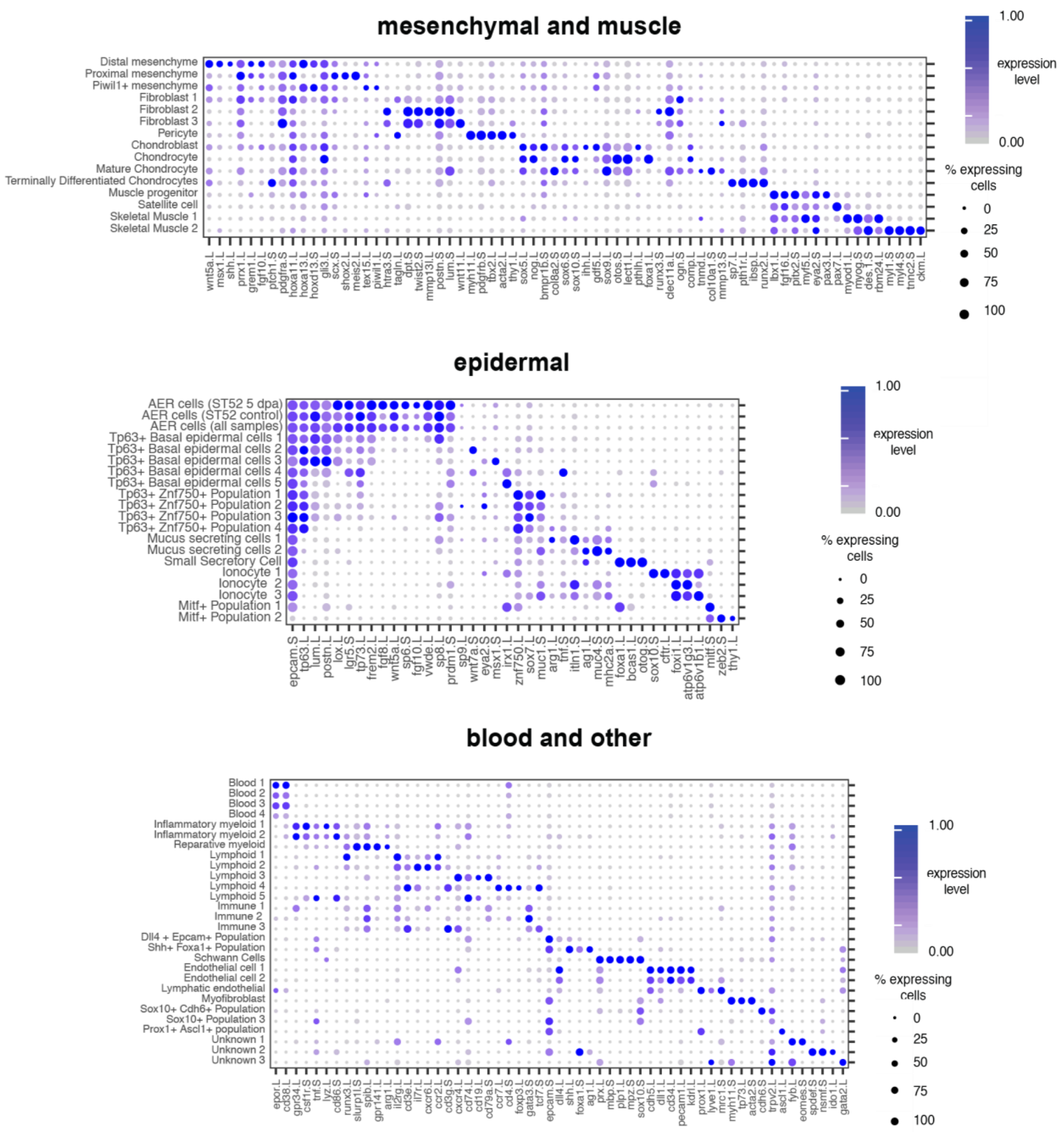

Figure S5: Annotation of cell types using known markers of cell identity.

Dotplots showing marker genes for each of the 61 cell types in our atlas. For ease of presentation, we group cell types into three broad categories: mesenchymal and muscle (top), epidermal (middle), blood and other (bottom). Please note that for the AER cell cluster, we also provide marker gene expression for specific samples as indicated. Dot color denotes mean expression level within the cluster; dot size denotes the percentage of cells within the cluster with non-zero expression. 
a
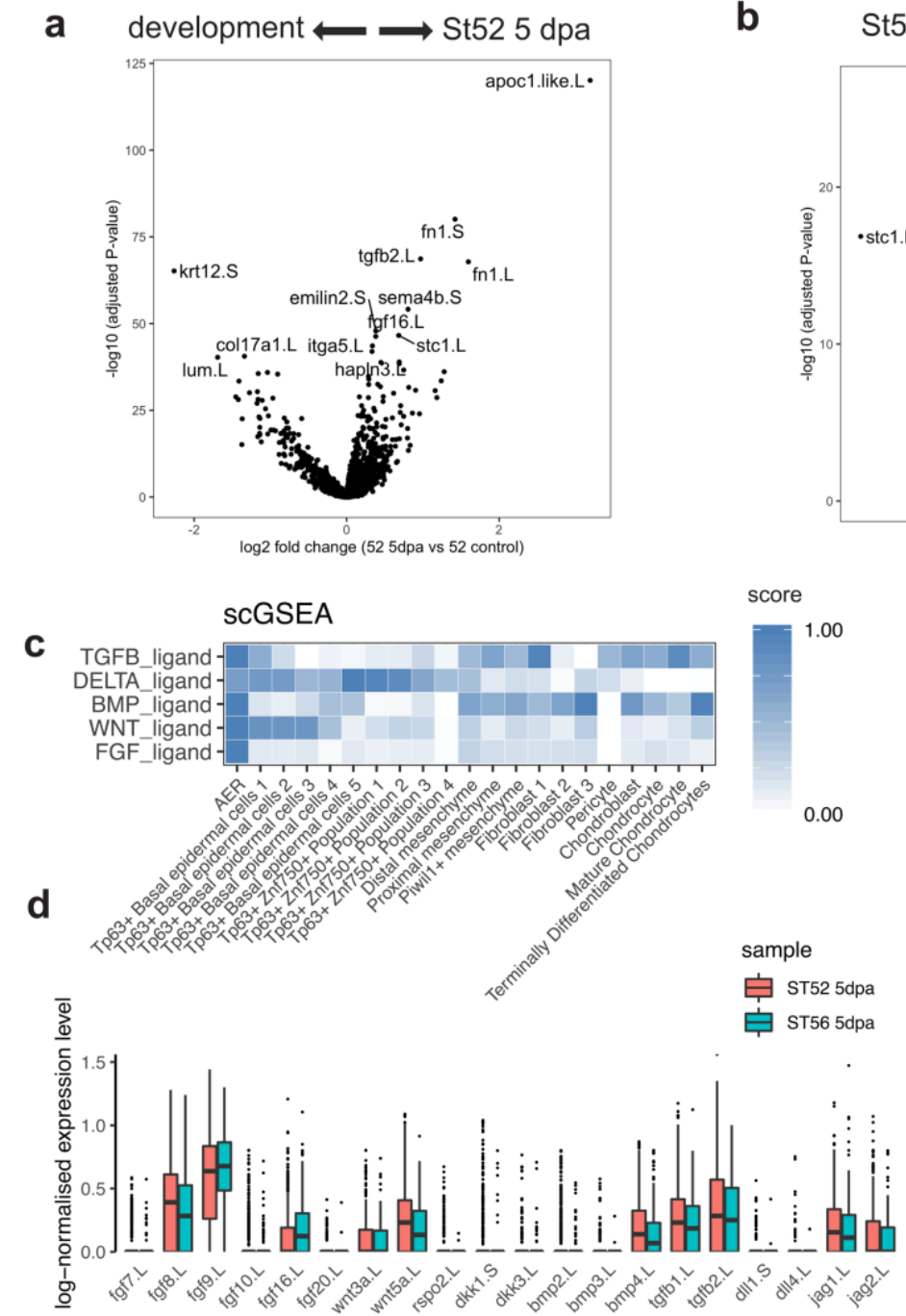

b

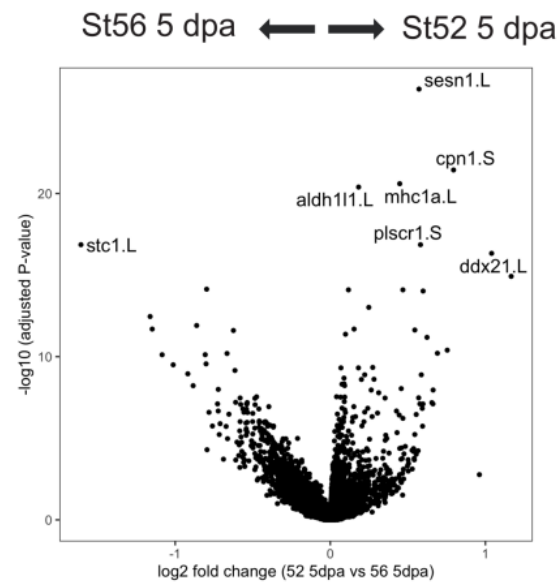

score
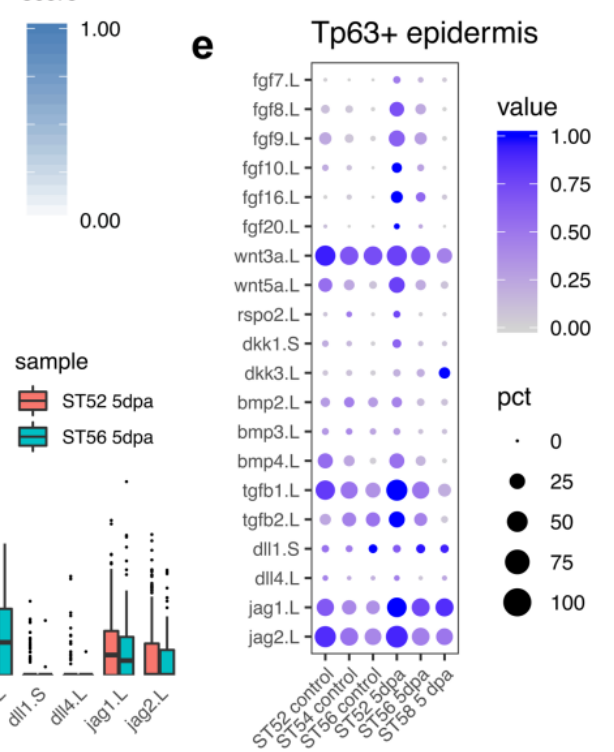

\section{Figure S6: AER cells are a signaling center population.}

Differentially expressed genes are detected in AER cells between pairs of conditions and visualised using volcano plots. In (a), Stage 525 dpa is compared to Stage 52 control samples; in (b), Stage 56 dpa is compared to Stage 52 ddpa samples. (c) Heatmap showing single-cell gene enrichment scores for ligands from the main signaling pathways are shown for epidermal cell types. AER cells have high signal center properties as they express high levels of TGF- $\beta$, Delta, BMP, WNT, and FGF ligands. Please see Supplementary Table 2 for the full list of ligands used in this analysis. (d) Log10-normalized gene expression visualised using boxplots to compare expression levels between Stage 525 dpa and Stage 565 dpa AER cells. (e) Dot plot showing expression of AER cell associated selected ligands for TP63+ epidermal cells during development and at $5 \mathrm{dpa}$ in regeneration-competent, -restricted, and -incompetent samples. Dot color indicates mean expression; dot size represents the percentage of cells with non-zero expression." 
a

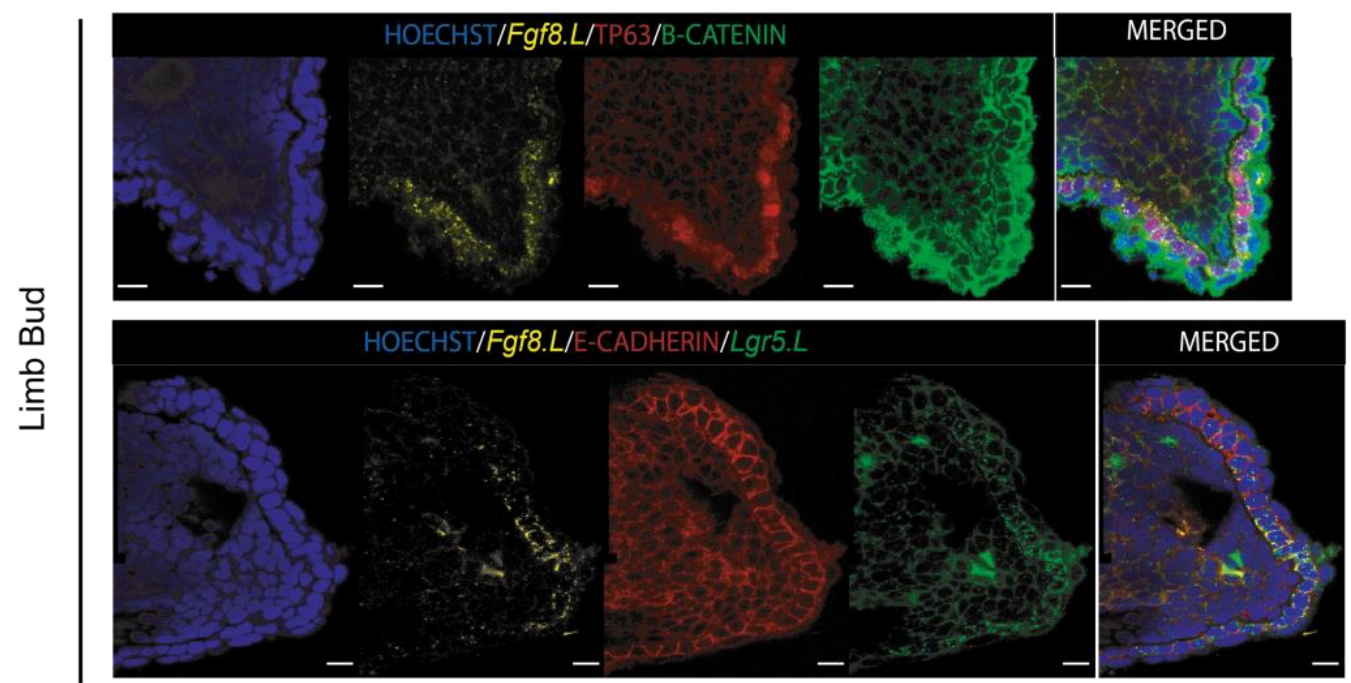

b

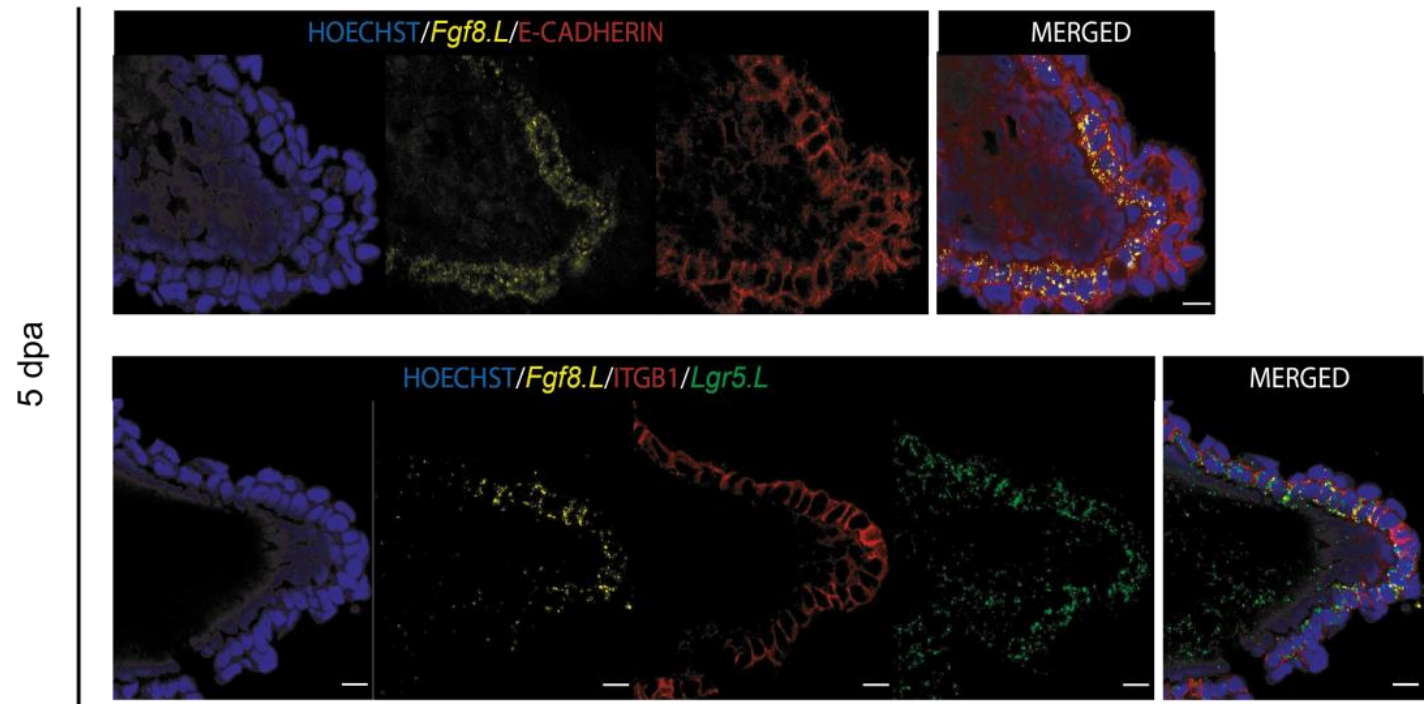

Figure S7: AER cells are largely found as cuboidal monolayer cells showing apical-basal polarity.

AER cells were visualized during limb development (a) and at 5 dpa (b) in regenerationcompetent tadpoles by labelling Fgf8.L mRNA. AER cells are largely present as monolayer cuboidal basal epidermal cells with apical-basal polarity. A simple squamous layer is present above AER cells, and cells with mesenchymal morphology are located underneath AER cells. From the proximal to distal midline of the epidermis, Lgr5.S expression is first detected, followed by Fgf8.L mRNA expression. Both Fgf8.L and Lgr5.S are expressed at high levels at the tip of limbs. AER cells show similar cuboidal morphology during development and regeneration. Basal epidermal cells are morphologically similar based on Hoechst and 
membrane markers, and Fgf8.L detection is required to detect AER cell. Row 1: Blue, Hoechst; Yellow, Fgf8.L mRNA; Red, TP63; Green, B-catenin. Row 2: Blue, Hoechst; Yellow, Fgf8.L mRNA; Red, E-Cadherin; Green, Lgr5.S mRNA. Row 3: Blue, Hoechst; Yellow, Fgf8.L mRNA; Red, E-Cadherin. Row 4: Blue, Hoechst; Yellow, Fgf8.L mRNA; Red, ITGB1; Green, Lgr5.S mRNA. Scale bars $=10 \mu \mathrm{m}$. 


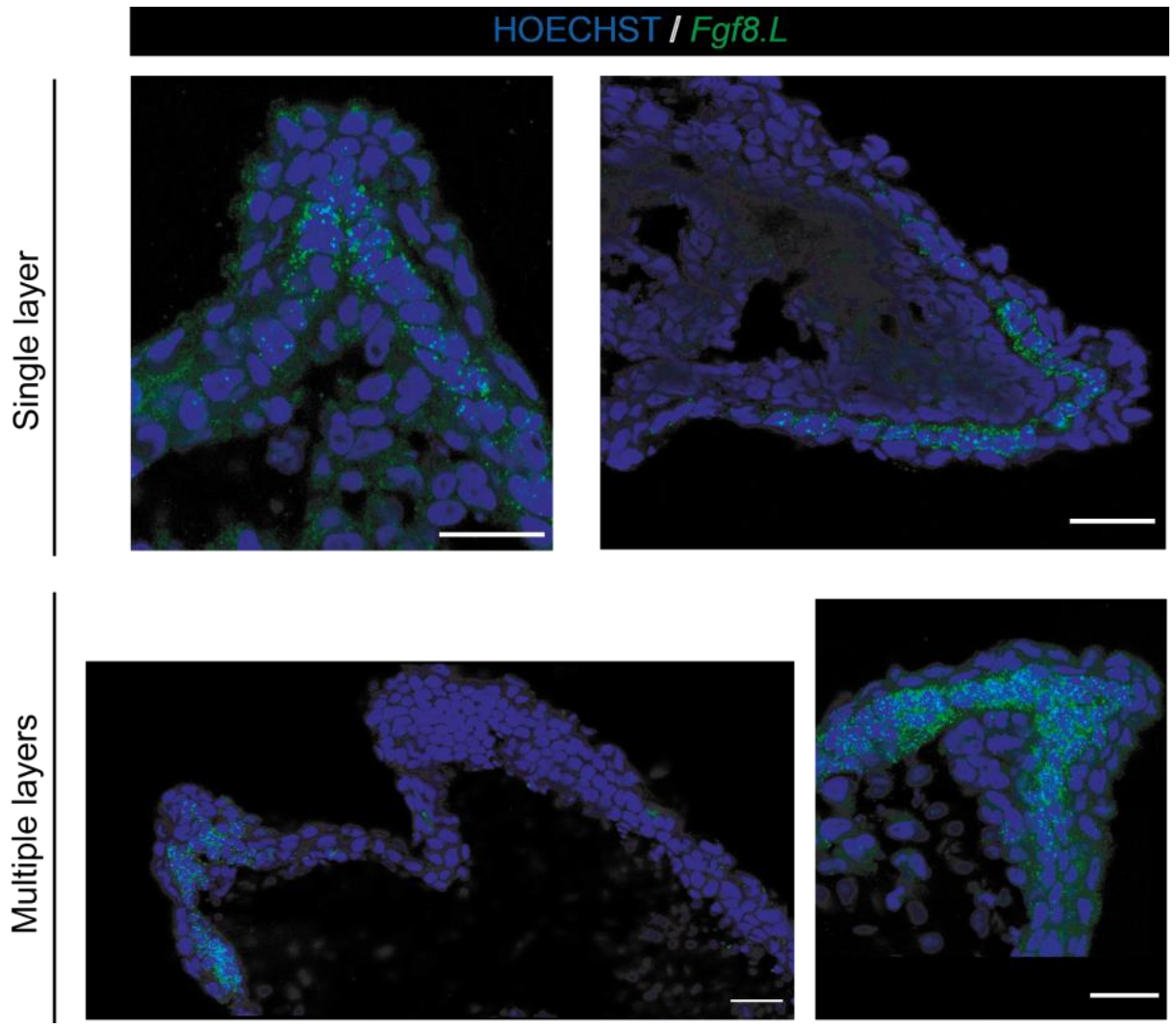

Figure S8: AER cells can be mono- or multi-layered structures.

Fgf8.L images of sectioned in vivo 5 dpa samples from regeneration -competent (top) and restricted (bottom) samples. Morphology of AER cells (Fgf8.L+) can vary between sections and samples. Top left, AER cells are seen as single monolayer largely cuboidal although some have higher height to width ratio. Top right, AER cells are seen as single monolayer largely cuboidal cells. Bottom left, AER cells can be seen as multi-layered population that is not covering the whole amputation plane. Bottom right, AER cells can be seen as multi-layered population covering the amputation plane. Blue, Hoechst; Green, Fgf8.L mRNA. Scale bars = $25 \mu \mathrm{m}$. 


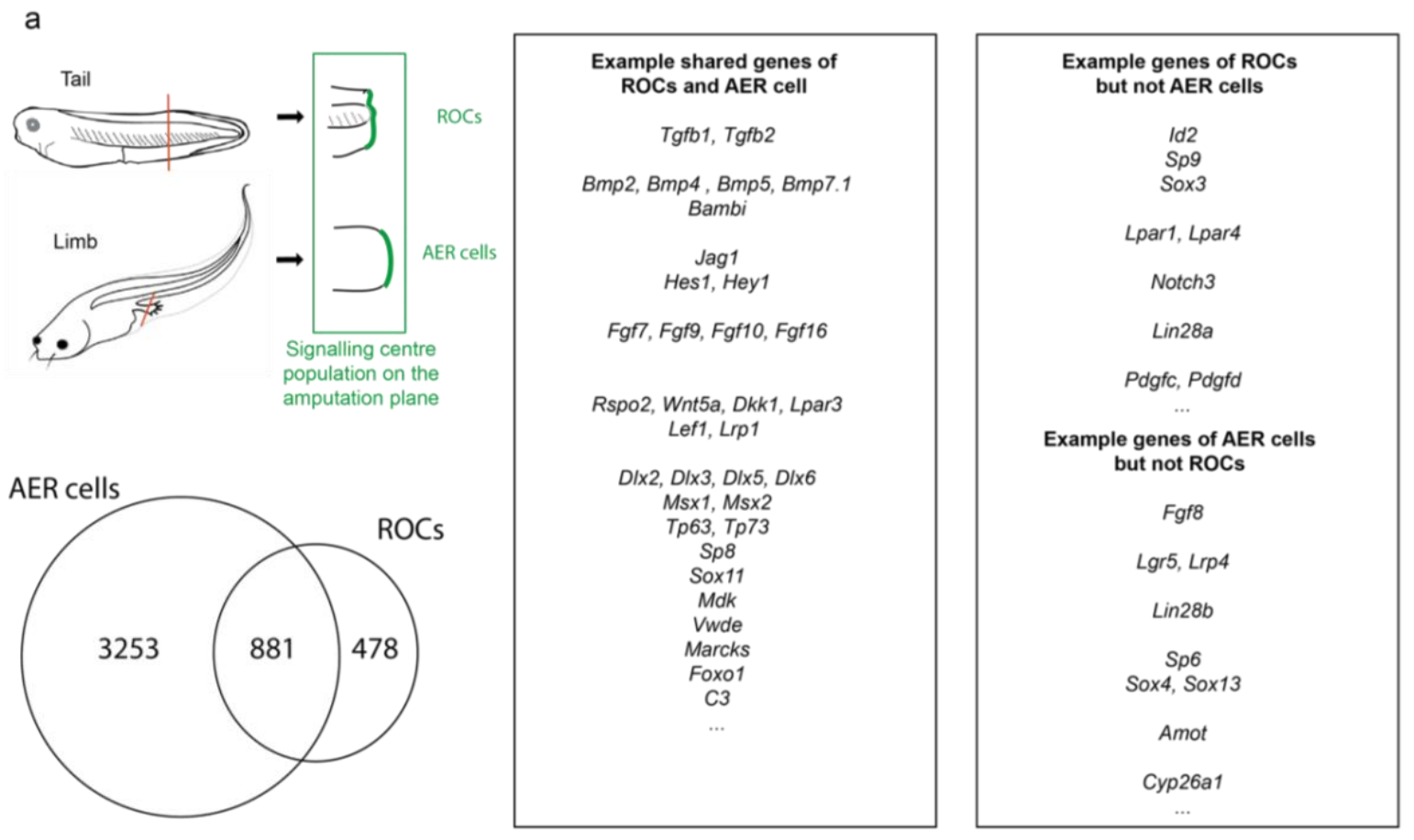

b

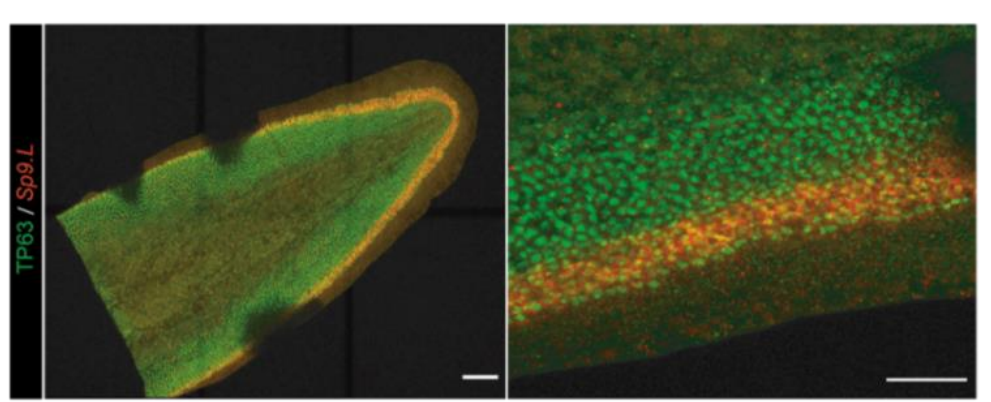

c
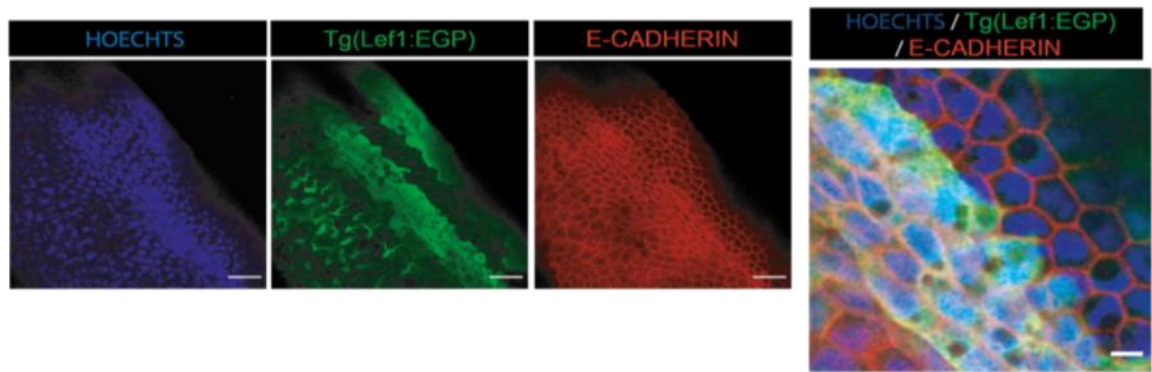

Figure S9: Specialised wound epidermis of tail and limb regeneration share some transcriptional similarities while presenting different cellular morphology.

(a) (Left) A signaling center population serving as the specialized wound epidermis is associated with Xenopus tail and limb regeneration. However, tail uses regenerationorganizing-cells (ROCs) (Aztekin et al., 2019) while limb uses AER cells for this purpose. Both AER cells and ROCs share the expression of many genes highlighting their similarity, although there are some genes that are unique to each population. AER- and ROC-specific genes were identified as genes significantly upregulated relative to other basal epidermal cells. (Right) A select number of genes, specifically ligands and transcription factors that are 
associated with regeneration, are highlighted. (b) ROCs and AER cells show different morphologies (please see Figure S7 for AER cells). ROCs were visualized by staining NF Stage 40 by Sp9.L mRNA expression (highly specific for ROCs (Aztekin et al., 2019)) and TP63 immunolabelling for whole tail (Left) and zoomed in version (Right). In the zoomed in version for staining Sp9.L shows two level of expression in ROCs: a single outer layer of $S p 9 . L$ low cells, and multiple inner layers of Sp9.L high cells. Please note that this is not whole bottom-top image of a tail as evidenced by absence of TP63 staining in the in middle part of the tissue. Red, Sp9.L mRNA; Green, TP63. Scale bars= (left) $250 \mu \mathrm{m}$, (right) $100 \mu \mathrm{m}$. (c) ROCs are visualized using the pbin7LEF:GFP line, as defined previously (Aztekin et al., 2019), and E-CADHERIN staining was used to delineate cell shape. Inner layers of ROCs have flattened cell shape while the outside layer ROCs exhibit more square-like shape. ROCs do not have branched nuclei, unlike fin cells. Blue, Hoechst; Green, EGFP; Red, E-cadherin. Scale bars $=10 \mu \mathrm{m}$. 

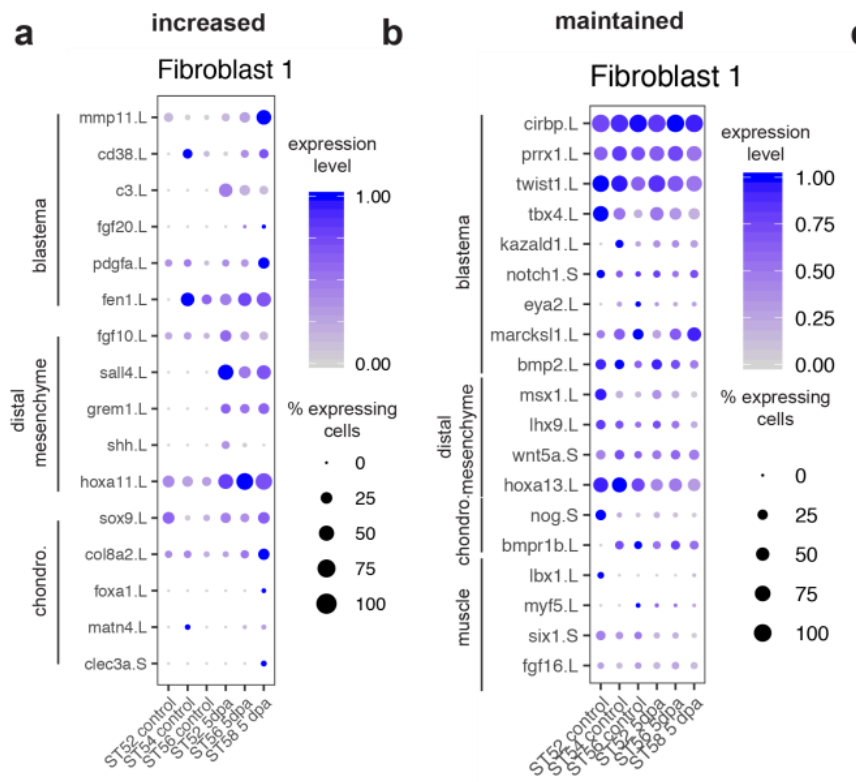

C

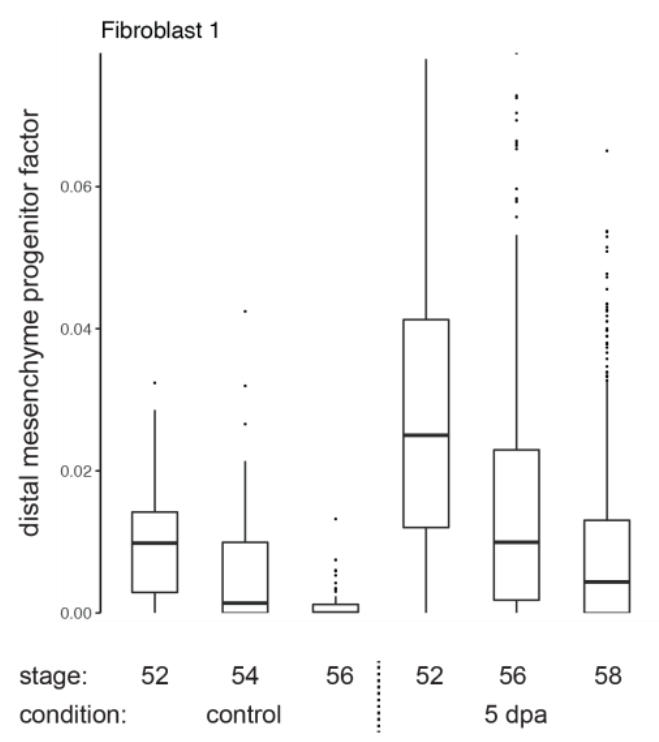

Figure S10: A subset of fibroblasts express dedifferentiation and blastema genes independently of the regeneration-outcome.

Expression of genes and putative gene sets associated with regeneration in the Fibroblast 1 cluster, visualized using dotplots and factor analysis. (a) Expression of specific genes that increase upon amputation regardless of stage. (b) Expression of specific genes that are expressed in intact limbs and are maintained after injury. (c) Following amputation, the putative distal mesenchyme progenitor gene set (factor) increases in Fibroblast 1 cells across all stages. 
a
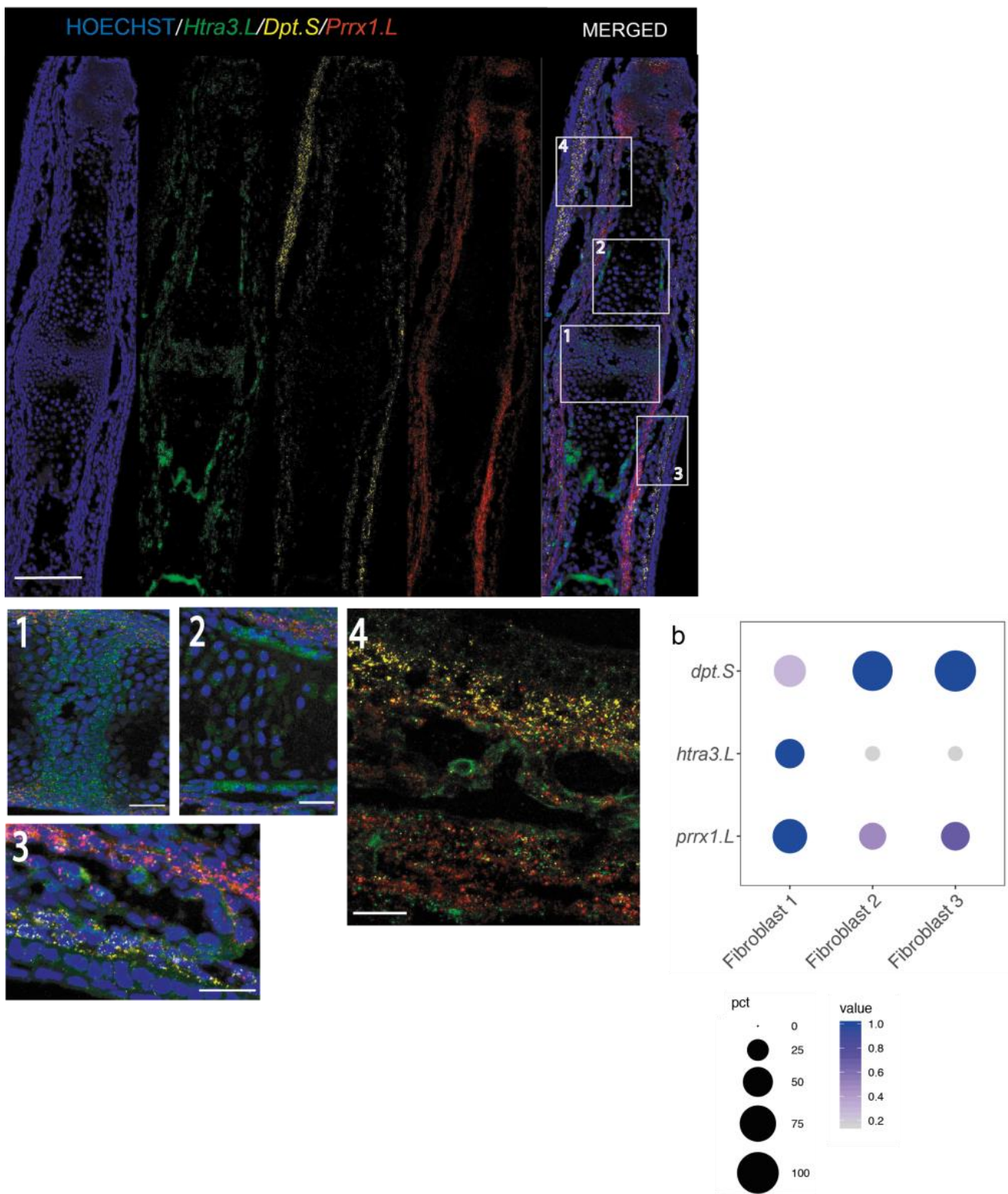

Figure S11: Fibroblast 1 cluster cells are largely found beneath skin cells and nearby perichondrial cells.

(a) (Top) Confocal images of a Stage 56 digit stained against Htra3.L, Prrxl.L, and Dpt.S. Cells expressing Htra3.L/Prrxl.L/Dpt.S are found underneath the skin regions and nearby perichondrium regions. (Bottom) Zoomed in version of selected areas show: (1) joint forming regions are enriched for Htra3.L expression; (2) Inner perichondrial regions are enriched for Htra3.L and outer perichondrial regions are enriched for Prrxl.L expression. (3-4) Outerlayers of dermal fibroblast area enriched for Dpt.S and lower levels of Prrxl.L and Htra3.L. Inner layers of dermal fibroblasts/nearby perichondrial regions are enriched for higher Prrx1.L and lower Dpt.S and Htra3.L expressions. Blue, Hoechst; Green, Htra3.L mRNA; Red, Prrx1.L mRNA; Yellow, Dpt.S mRNA. Scale= $125 \mu \mathrm{m}$ for top images, $25 \mu \mathrm{m}$ for bottom no 1-3, and 
$20 \mu \mathrm{m}$ for bottom no 4. (b) Dot plot showing expression of Htra3.L, Prrx1.L, and Dpt.S for Fibroblast 1, 2, and 3 clusters. 
a

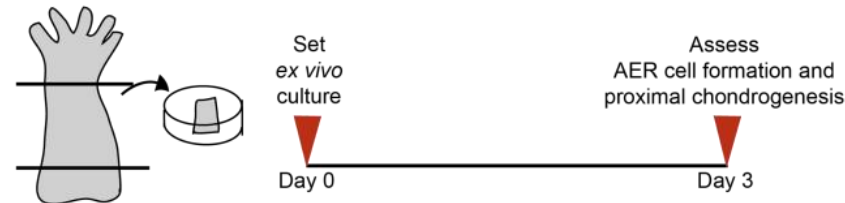

b Length of the Fgf8.L+ population on the amputation plane (mm)

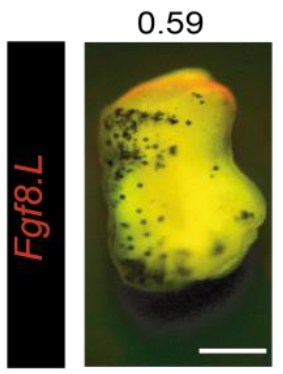

0.41

0.38

0.16
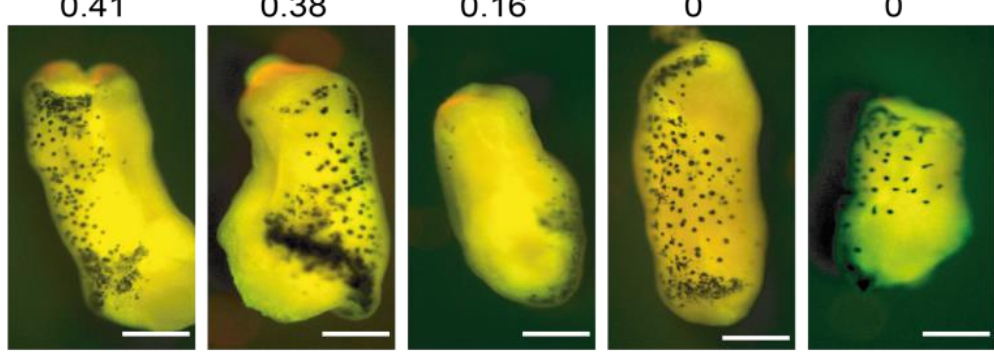

C Length of the proximal site chondrogenesis $(\mathrm{mm})$

0.58

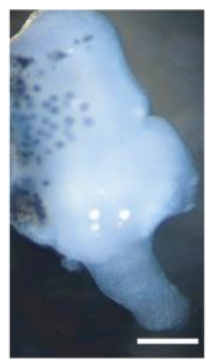

d

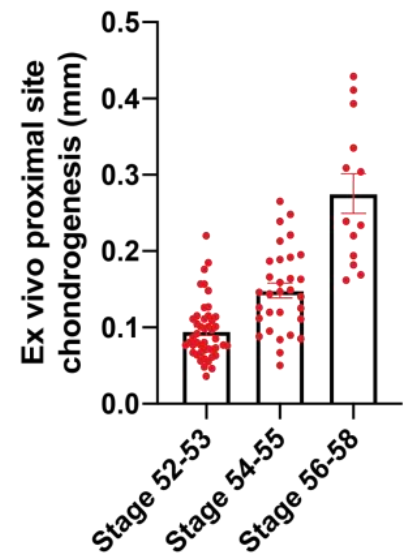

0.28

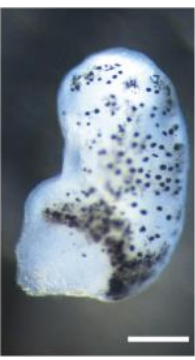

e
0.21

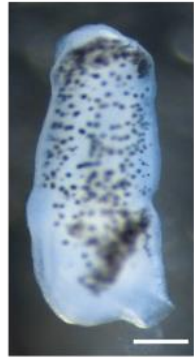

0.15

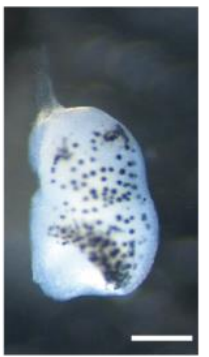

0.13

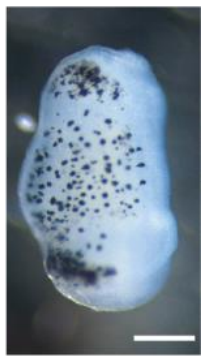

Figure S12: The distal site of ex vivo regenerating limbs can be used to detect AER cell formation, and the proximal site of explants can be used for detecting chondrogenesis.

(a) Schematics describing the ex vivo culture protocol for assessing Fgf8.L mRNA expression at the distal site, and chondrogenesis levels at the proximal site. All assessments were carried out at 3-days post culture start. (b) Whole-mount stereomicroscope images of Fgf8.L stained limb explants at $3 \mathrm{dpa}$. Numbers at the top indicates AER cell formation measured as the length of Fgf8.L+ signal on the amputation plane. Red, Fgf8.L mRNA. Scale $=200 \mu \mathrm{m}$. (c) Wholemount stereomicroscope images of chondrogenesis at the proximal site of explants at $3 \mathrm{dpa}$. 
Numbers at the top indicates the measured proximal chondrogenesis extent. Scale $=200 \mu \mathrm{m}$. (d) Ex vivo regenerating limb cultures can be used to investigate chondrogenesis. Explants were cultured for 3 days and chondrogenesis was measured as in (c). The extent of chondrogenesis seen at the proximal site of explants changes with the developmental stage and coincides with the progression of in vivo chondrogenesis (Dent, 1962). Regenerationcompetent explants= total 46 samples from 4 biological replicates; Regeneration-restricted explants $=$ total 31 samples from 3 biological replicates; Regeneration-incompetent explants= total 13 samples from 3 biological replicates. $P^{* *}<0.001$. (e) Explants were cultured for 3 days with BMP4 and the extent of chondrogenesis was measured. Addition of recombinant BMP4 to the explant media increased the observed chondrogenesis at the proximal site. Control $0.1 \%$ BSA, total $n=16$ samples from 4 biological replicates; recombinant BMP4, total $n=16$ samples from 4 biological replicates. 
a
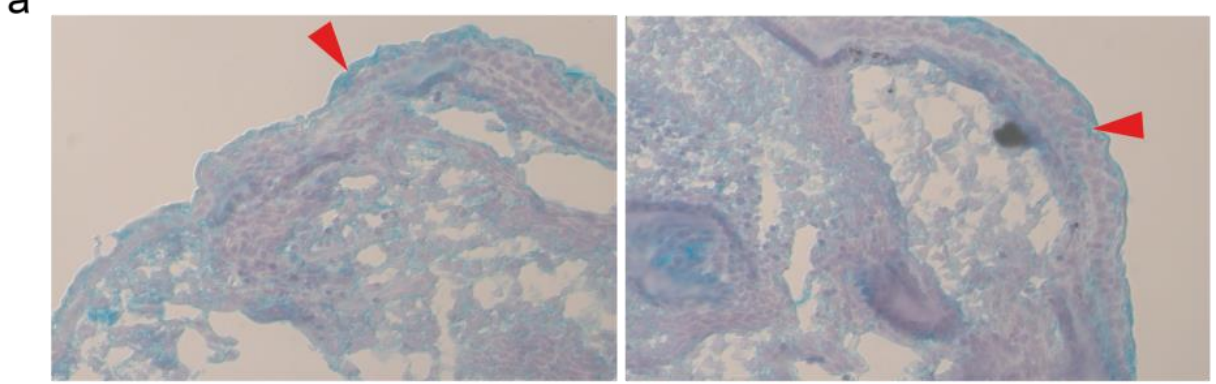

b
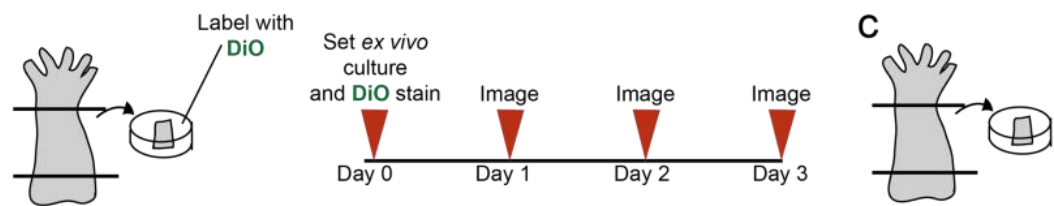

0 dpa

1 dpa

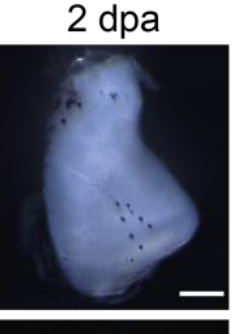

3 dpa
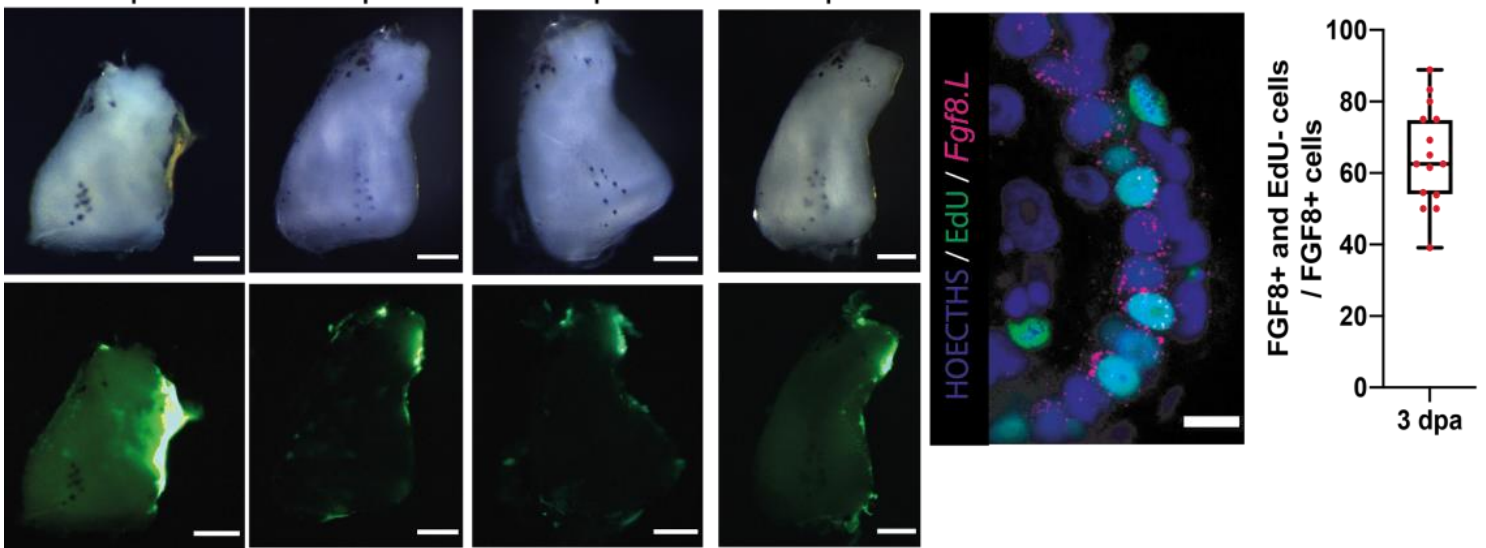

Figure S13: AER cells formation does not require cell division.

(a) 3 dpa regeneration-competent explants are covered with cells morphologically similar to the surrounding basal epidermal cells as evidenced by haematoxylin, eosin, and Alcian blue stain. There are multi-layered or monolayered epidermal cells with cuboidal shape that can be seen not only at the distal site (right-bottom) but also at the lateral sides as well (right-top). A squamous layer can be seen above the basal epidermal cells. (b) (Top) Schematic describing $\mathrm{DiO}$ based tissue tracing. DiO labelling was performed after ex vivo cultures were harvested. Explants were imaged every day until day 3 in culture via stereomicroscope. (Bottom) DiO tracing applied to the sides of explants and traced over time and images were taken in brightfield and green channel. Traced tissues migrated to the distal and proximal amputation planes of explants. Total $n=22$ from 2 biological replicates. Scale $=200 \mu \mathrm{m}$. (c) (Top) 
Schematics describing ex vivo culture with EdU treatment. EdU was added to explant media at the beginning of the culture. (Bottom-left) Explants were fixed and stained via HCR for Fgf8.L, and EdU after day 3 in culture. (Bottom-middle) Example confocal image of a sectioned sample stained via HCR for Fgf8.L, Lgr5.S, and EdU, showing that not all Fgf8.L+/Lgr5.S+ cells are EdU+. Hoechst, Blue; EdU, Green, Fgf8.L mRNA, Magenta. Scale $=10 \mu \mathrm{m}$. (BottomRight) Quantification of EdU positive AER cells proportion to all detected AER cells. Approximately $60 \%$ of AER cells are EdU negative. Total $n=15$ from 3 biological replicates. 


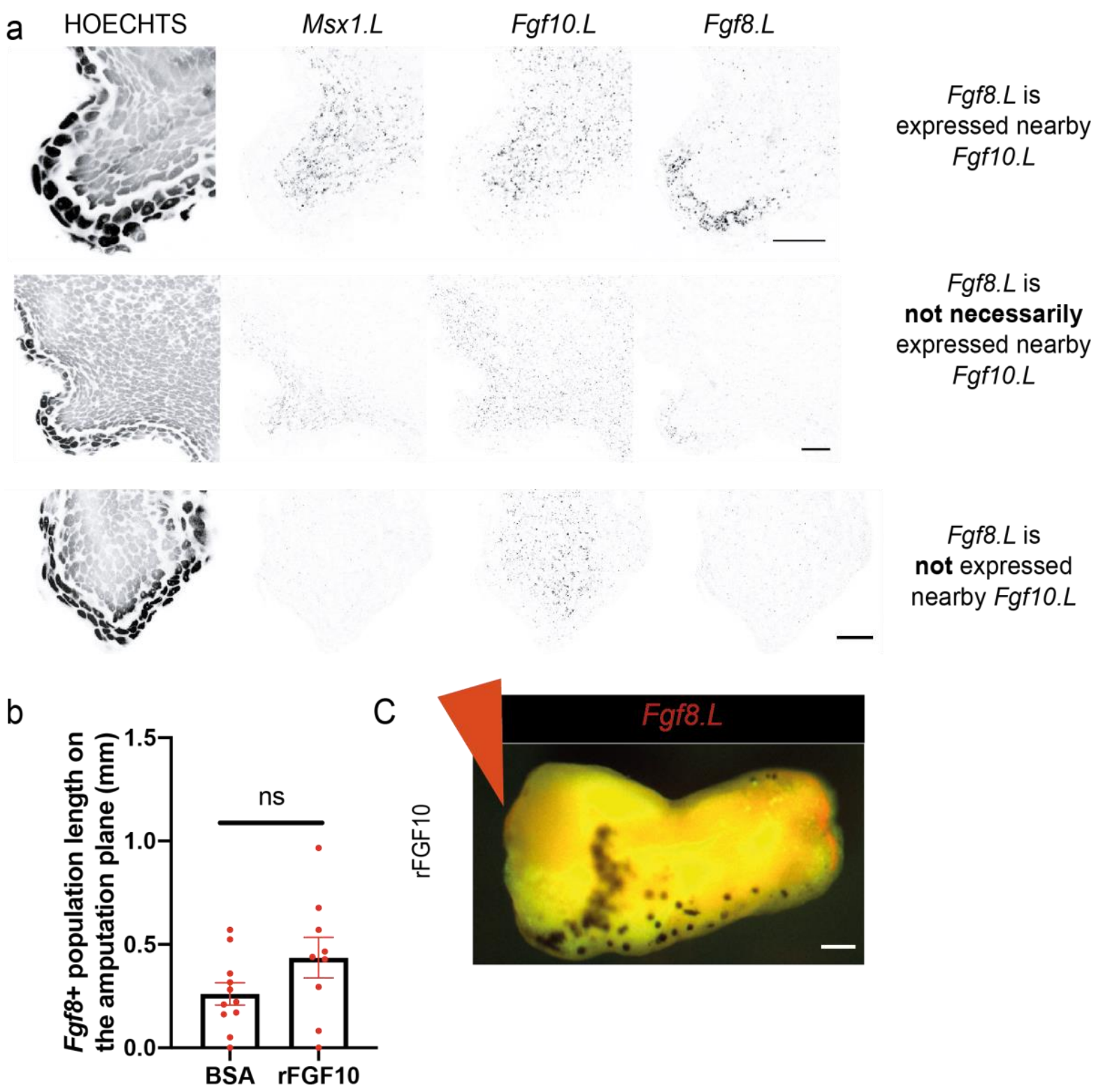

Figure S14: FGF10 is not sufficient to induce $F g f 8$ in all epidermis.

(a) Examples of sectioned confocal images of 5 dpa in vivo samples from regenerationcompetent tadpoles stained for Msxl.L, Fgf10.L and Fgf8.L. Top image series show high levels of Fgf10.L and Msxl.L in the mesenchyme associated with high levels of Fgf8.L in the surrounding epidermis. Middle image series show that not all epidermis in proximity of Fgf10.L + mesenchymal cells are expressing Fgf8.L. Msxl.L + mesenchymal cells are more correlated to Fgf8.L+ epidermis than FgflO.L + mesenchymal cells. Bottom, although there is a high level of Fgf10 expression detected in mesenchyme, no Fgf8.L in epidermis or Msx1.L in mesenchyme can be seen. Scale, $20 \mu \mathrm{m}$. (b) Regeneration-competent explants were treated with $\mathrm{rFGF} 10$, and $0.1 \%$ BSA/PBS was used as control. BSA: total $\mathrm{n}=11$ from 2 biological replicates; FGF10: total $\mathrm{n}=9$ from 2 biological replicates; ns: $P^{*}>0.05$. (c) Example whole- 
mount stereomicroscope image of rFGF10 treated explants showing a very mild expression of $F g f 8 . L$ at their proximal sites ( $\mathrm{n}=4 / 7$ from 2 biological replicates). Scale, $200 \mu \mathrm{m}$. 

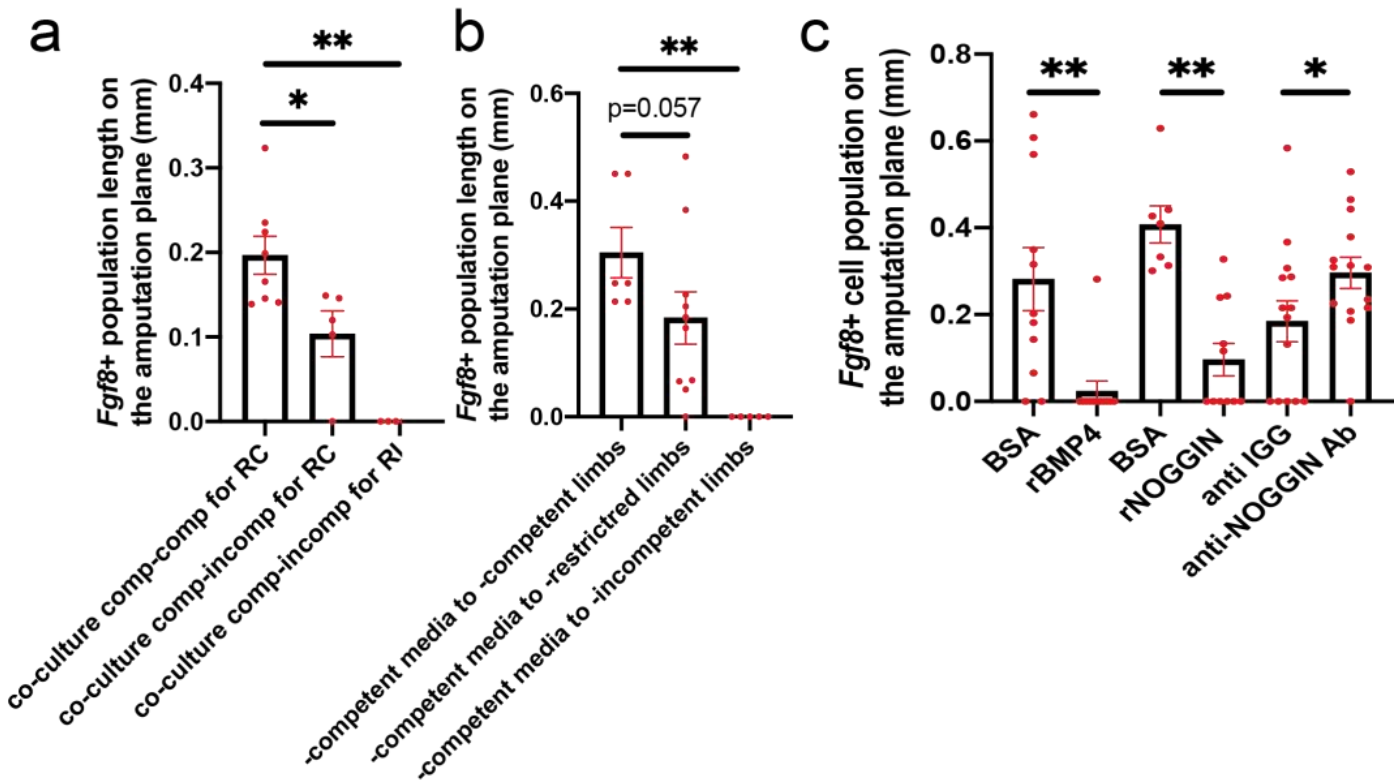

d

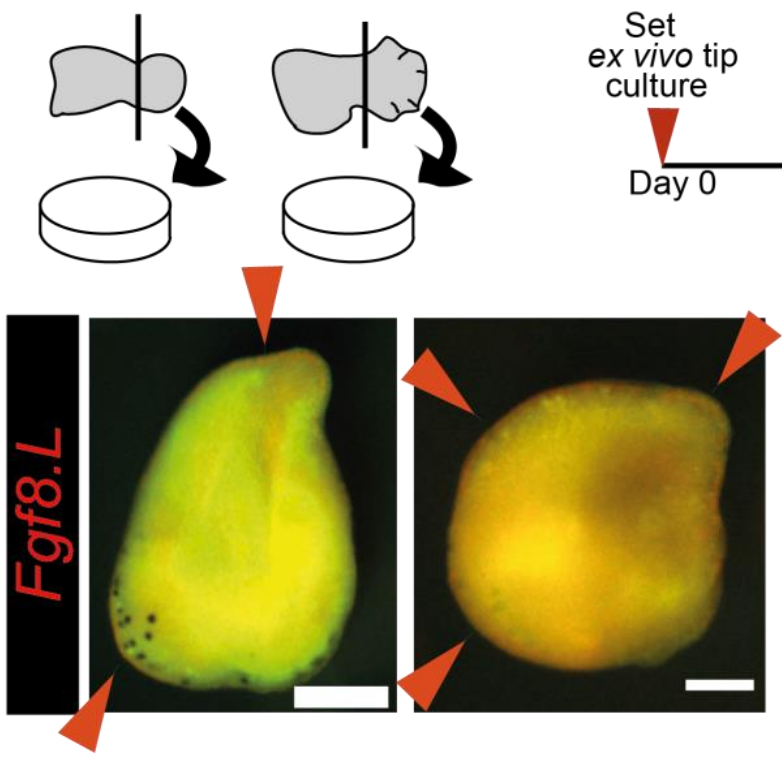

Set culture

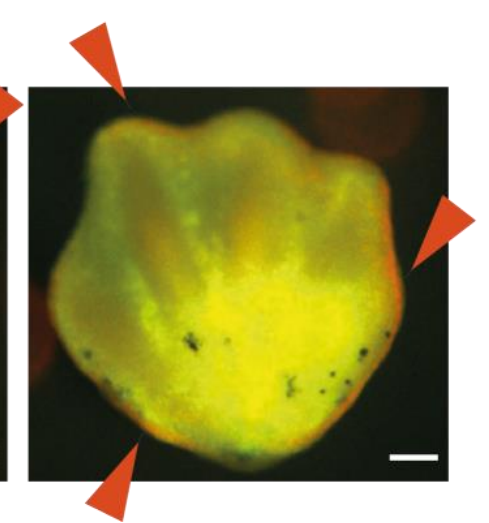

Figure S15: Secreted factors from regeneration-competent explants are not sufficient to induce $\mathrm{Fg} f 8+$ epidermal cells on the amputation plane of regeneration-incompetent explants, and reducing the proportion of chondrogenic lineage populations in explants can induce ectopic $F g f 8+$ epidermal cells.

(a) Co-culturing regeneration-competent with -incompetent explants does not enable AER cell formation ability in -incompetent explants. RC: regeneration-competent; RI: regenerationincompetent. Co-culture of regeneration-competent-competent and assess -competent: total $\mathrm{n}=8$, from 2 biological replicates, Co-culture regeneration-competent-incompetent and assess -competent: $\mathrm{n}=5$ from 2 biological replicates. Co-culture regeneration-competent-incompetent for -incompetent total $\mathrm{n}=6$ from 2 biological replicates. $P^{*}<0.05$, and $P^{* *}<0.001$. (b) Treatment with -competent conditioned media does not enable AER cell formation in - 
incompetent explants. Adding -competent-media to -competent explants: total $n=6$, from 2 biological replicates. Adding -competent-media to -restricted explants: $n=10$, from 3 biological replicates. Adding -competent-media to -incompetent explants: $n=5$, from 1 biological replicate. $P^{* *}<0.001$. (c) Regeneration-competent explants were treated with recombinant BMP4, recombinant NOGGIN, or anti-NOGGIN antibodies. Contralateral limbs were used as controls and treated with vehicle solutions (0.1\% BSA, or anti-IGG). Recombinant BMP4 or NOGGIN additions block AER cell formation. Anti-NOGGIN antibody treatment enhances AER cell formation. From left to right $0.1 \%$ BSA: total $n=11$ from 3 biological replicates; rBMP4: total $n=12$ from 3 biological replicates; $0.1 \%$ BSA: total $n=7$ from 2 biological replicates; rNOGGIN: total $\mathrm{n}=11$ from 2 biological replicates; anti-IGG antibody: total $n=14$ from 4 biological replicates; anti-NOGGIN antibody: total $n=14$ from 4 biological replicates. Each sample group compared to their contralateral group to assess statistical significance. $P^{*}<0.05$, and $P^{* *}<0.001$. (d) (Top) Schematic describing the protocol for culturing distal limb buds (NF stage $~ 52$ ) and early autopods (NF Stage 54). Tip explants were cultured for 3 days in explant media, and assessed for Fgf8.L expression. (Bottom) Tip cultures show ectopic Fgf8.L expression as assessed by whole-mount stereomicroscope HCR staining for Fgf8.L. Red arrows show Fgf8.L expression regions. Ectopic AER formation is seen in total 16/18 cases from 2 biological replicates. Red, Fgf8. $L$ mRNA. Scale $=100 \mu \mathrm{m}$. 


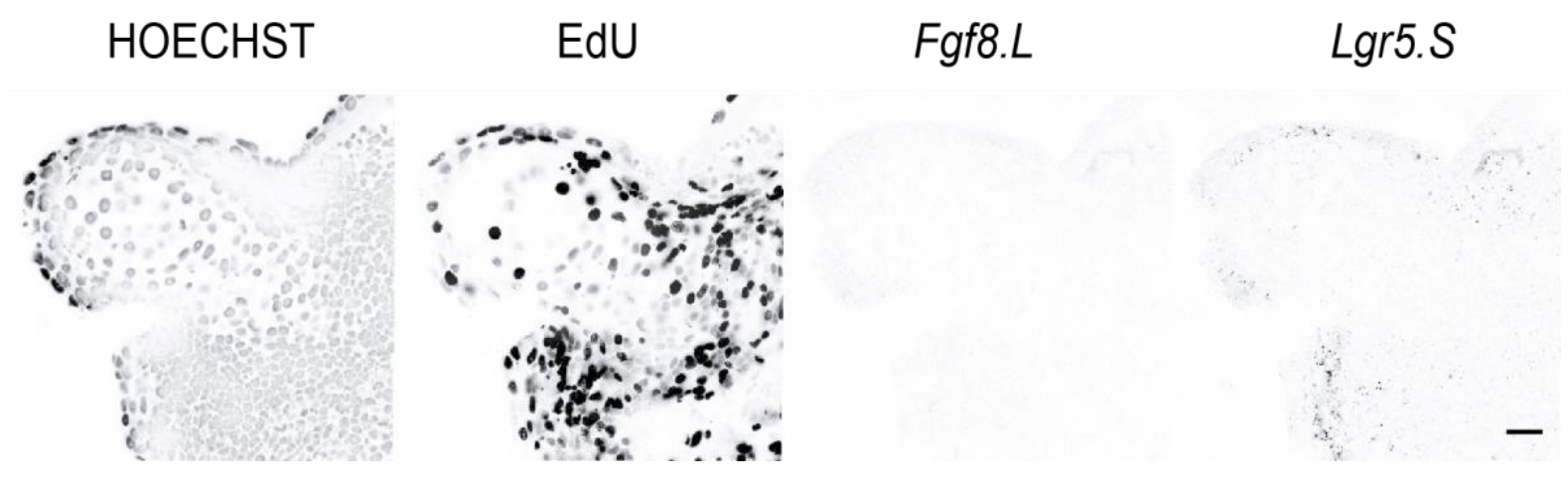

Figure S16: Inhibition of FGF receptor induces chondrogenic populations in the proximal explant.

Example sectioned confocal images of proximal site of 3 dpa explants treated with SU5402 showing sparse circular nucleus indicative of chondrogenic cells as well as lack of Fgf8.L in epidermis. Scale bar $=15 \mu \mathrm{m}$.

Table S1. Quality control for scRNA-seq

Click here to download Table S1

Table S2. Differentially expressed gene lists for AER cells during development and regeneration, and ligand gene lists used for scGSEA.

Click here to download Table S2 